Supporting Information for the Paper Entitled:

\title{
Uranium Going the Soft Way: Low-Valent Uranium(III) Coordinated to an Arene-Anchored Tris-Thiophenolate Ligand
}

\author{
Daniel Pividori, ${ }^{\dagger}$ Matthias E. Miehlich, ${ }^{\dagger}$ Benedikt Kestel,${ }^{\dagger}$ Frank W. Heinemann, ${ }^{\dagger}$ Andreas \\ Scheurer, ${ }^{\dagger}$ Michael Patzschke, ${ }^{\star}$ and Karsten Meyer*† \\ ${ }^{\dagger}$ Friedrich-Alexander-University of Erlangen-Nürnberg (FAU), Department of Chemistry and \\ Pharmacy, Inorganic Chemistry, Egerlandstraße 1, 91058 Erlangen, Germany \\ $¥$ Institute of Resource Ecology, Helmholtz-Zentrum Dresden-Rossendorf, Bautzner Landstraße 400, \\ 01328 Dresden, Germany
}

\section{Supporting Information Table of Contents}

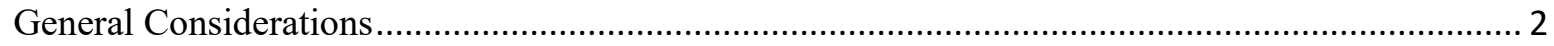

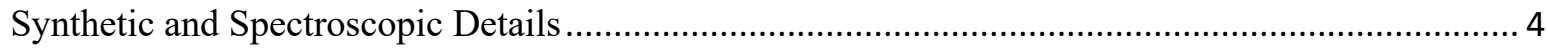

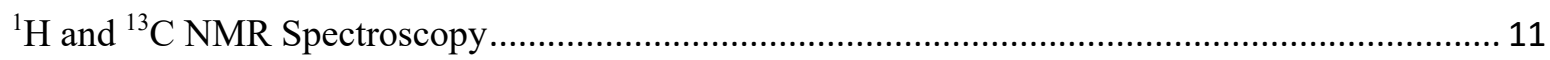

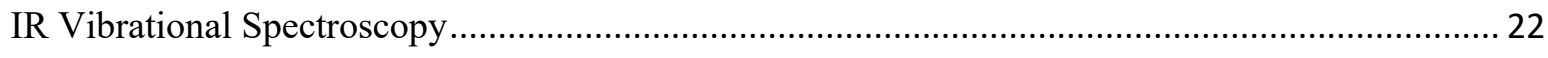

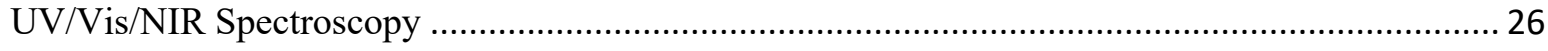

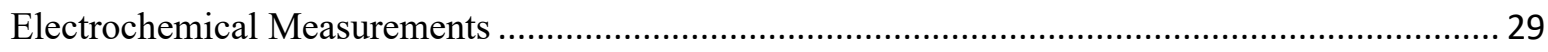

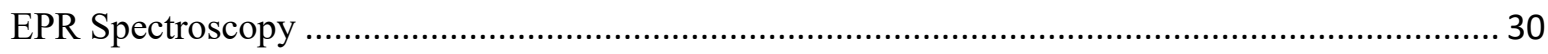

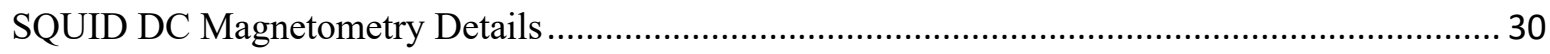

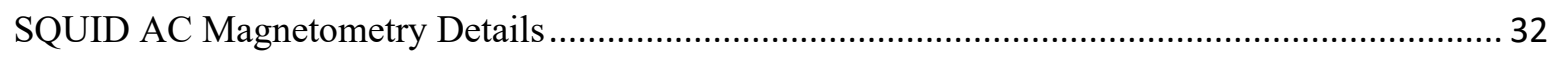

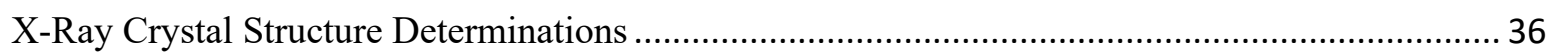

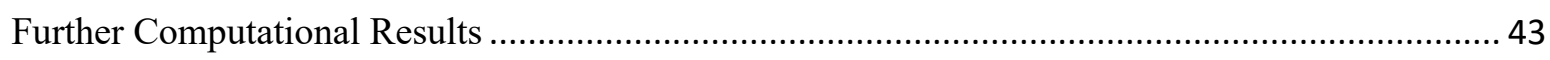

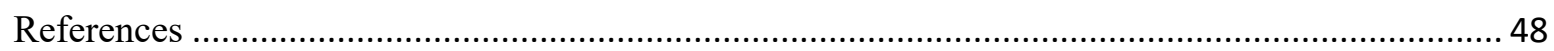




\section{General Considerations}

All air- and moisture-sensitive experiments were performed under dry Argon atmosphere, using standard Schlenk techniques or an MBraun inert-gas glovebox, containing an atmosphere of purified dinitrogen. The glovebox was equipped with a $-30{ }^{\circ} \mathrm{C}$ freezer. Solvents were purified using a twocolumn solid-state purification system (Glass Contour System, Irvine, CA), transferred to the glovebox without exposure to air and moisture, and stored over activated molecular sieves and potassium, where appropriate ( $n$-pentane, $n$-hexane, benzene). Commercially available starting materials were purchased from prominent suppliers (Acros Organics, Alfa Aesar, Sigma Aldrich, Merck, TCI, VWR) and were used without further purification. The reagents 2-adamantyl-4-methylphenol ${ }^{1}$ and $\left[\mathrm{U}\left(\mathrm{N}\left(\mathrm{SiMe}_{3}\right)_{2}\right)_{3}\right]^{2}$ were prepared according to literature procedures. NMR solvents were obtained packaged under argon and stored over activated molecular sieves.

NMR spectra were recorded on a JEOL ECX $400 \mathrm{MHz}$ instrument operating at a frequency of $400.18 \mathrm{MHz}\left({ }^{1} \mathrm{H}\right.$ NMR $), 100.6 \mathrm{MHz}\left({ }^{13} \mathrm{C}\right.$ NMR $)$, with a probe temperature of $23{ }^{\circ} \mathrm{C}$. Chemical shifts, $\delta$, are reported relative to the ${ }^{1} \mathrm{H}$ or ${ }^{13} \mathrm{C}$ resonances of the solvent in ppm. ${ }^{3}$ NMR multiplicities are abbreviated as follows: $\mathrm{s}=$ singlet, $\mathrm{d}=$ doublet, $\mathrm{t}=$ triplet, $\mathrm{m}=$ multiplet, $\mathrm{br}=$ broad signal.

Electronic absorption spectra were recorded from $\lambda=200 \mathrm{~nm}$ to $\lambda=2500 \mathrm{~nm}$ on a Shimadzu double beam UV-3600 UV/Vis/NIR spectrophotometer in given solvents at room temperature. Cuvettes of the type 117.100-QS from Hellma with 10 mm layer thickness were used.

Electrochemical measurements were carried out at room temperature under inert gas atmosphere with an Autolab Type-III potentiostat, recorded in $0.1 \mathrm{M} \mathrm{n}$-Bu $\mathrm{NPF}_{6}\left(\mathrm{TBAPF}_{6}\right.$, purchased from Acros and used without further purification) solution in THF, using a glassy carbon working electrode and $\mathrm{Pt}$ electrodes as counter and pseudo-reference electrodes. Ferrocene was added as internal standard and all measurements were referenced to $\mathrm{E}\left(\mathrm{Fc} / \mathrm{Fc}^{+}\right)=0.41 \mathrm{~V} v s$. NHE.

Infrared (IR) spectra were recorded on a Shimadzu Affinity-1 system in KBr pellets, or on a Shimadzu IRSpirit QATR-S system as a neat powder.

Magnetism data of powdered samples (20.2-26.1 mg), loaded within polycarbonate gel capsules, were collected on a Quantum Design MPMS-3 SQUID magnetometer. DC susceptibility was recorded in the temperature range of 2-300 K with an applied DC field of $1 \mathrm{~T}$, if not stated otherwise. Values of the magnetic susceptibility were corrected for core diamagnetism of the sample using tabulated Pascal's constants $\left(\chi_{\text {dia }}=-700.11 \mathrm{~cm}^{3} \mathrm{~mol}^{-1}\right.$ for $\left.\mathbf{1}-\mathbf{U}\right){ }^{4}$

Alternating current (ac) magnetism data were recorded in the temperature range of $1.8-11 \mathrm{~K}$ with an applied field of $0-0.3 \mathrm{~T}$, ac amplitude of $3 \mathrm{Oe}$, and ac frequency of $1-941 \mathrm{~Hz}(941 \mathrm{~Hz}$ is the maximal value at an amplitude of $3 \mathrm{Oe}$ ). The Cole-Cole (Argand) plots were fitted to the generalized Debye model: ${ }^{5}$ 


$$
\chi(\omega)=\chi^{\prime}(\omega)-i \chi^{\prime \prime}(\omega)=\chi_{S}+\frac{\left(\chi_{T}-\chi_{S}\right)}{1+(i \omega \tau)^{1-\alpha}}
$$

where $\omega$ is the angular frequency $\left(2 \pi v_{\mathrm{ac}}\right), \chi_{T}$ is the isothermal susceptibility, $\chi_{S}$ is the adiabatic susceptibility, $\tau$ is the average relaxation time, and the parameter $\alpha(0 \leq \alpha<1)$ describes the distribution of relaxation times.

The relaxation rate of the magnetization, $\tau^{-1}$, was fitted as a sum of all possible molecular relaxation processes: ${ }^{5,6}$

$$
\tau^{-1}(T)=\tau_{Q T M}^{-1}+A T+C T^{n}+\tau_{0}^{-1} \exp \left(-\frac{U_{e f f}}{k_{B} T}\right)
$$

where $\tau_{Q T M}^{-1}, \mathrm{~A}, \mathrm{C}$, and $\tau_{0}^{-1}$ are parameters describing the involved relaxation processes (quantum tunneling of magnetization, direct relaxation, Raman relaxation, and Orbach relaxation, respectively), and are taken as free fit parameters. The Raman exponent, $n$, is predicted as $n=9$ for a Kramers ion, but different values are possible in practice. ${ }^{6,7}$

Elemental analyses were obtained using Euro EA 3000 (Euro Vector) and EA 1108 (Carlo-Erba) elemental analyzers in the Chair of Inorganic and General Chemistry at the Friedrich-AlexanderUniversity Erlangen-Nürnberg (FAU). Commercial laboratories refuse samples that contain fluorine as it destroys modern elemental analyzers. Besides this practical problem, fluorine often causes substantial variations from the expected masses. ${ }^{8}$

EPR spectra were recorded on a JEOL continuous wave spectrometer JES-FA200, equipped with an $\mathrm{X}$-band Gunn diode oscillator bridge, a cylindric mode cavity, and a helium cryostat. The samples were measured in the solid state under nitrogen atmosphere in quartz glass EPR tubes at $7 \mathrm{~K}$. The spectra shown were measured using the following parameters: microwave frequency $=8.959 \mathrm{GHz}$, modulation width $1.0 \mathrm{mT}$, microwave power $1.0 \mathrm{~mW}$, modulation frequency $100 \mathrm{kHz}$, time constant of $0.1 \mathrm{~s}$. Data analysis and simulation of the data was performed using the software "eview" and "esim", written by Dr. Eckhard Bill (MPI CEC, Mülheim/Ruhr), ${ }^{9,10}$ on the basis of a spin-Hamiltonian description of the electronic ground state:

$$
\widehat{H}=D\left(\hat{S}_{z}^{2}-\frac{1}{3} S(S+1)+\frac{E}{D}\left(\hat{S}_{x}^{2}-\hat{S}_{y}^{2}\right)\right)+\mu_{B} \underline{g} \vec{S}
$$

Here, $S$ represents the total spin quantum number of the coupled system, $D$ and $E / D$ are the axial and rhombic zero-field parameters, respectively, and $g$ is the g-matrix. Calculations are based on the $S=$ $5 / 2$ routines developed by Gaffney and Silverstone. ${ }^{11}$ EPR line widths, $W$, are given in units of $\mathrm{mT}$ at full-width-half-maximum (FWHM).

Single-crystal X-ray structure data: Suitable single crystals of the investigated compounds were embedded in protective perfluoropolyalkyether oil on a microscope slide and a single specimen was 
selected and subsequently transferred to the cold nitrogen gas stream of the diffractometer. Intensity data were collected using $\operatorname{Mo} K_{\alpha}$ radiation $(\lambda=0.71073 \AA)$ on a Bruker Kappa PHOTON $2 I \mu S$ Duo diffractometer equipped with QUAZAR focusing Montel optics. Data were corrected for Lorentz and polarization effects, semiempirical absorption corrections were performed on the basis of multiple scans using SADABS. ${ }^{12}$ The structures were solved by direct methods (SHELXXT 2014/5) $)^{13}$ and refined by full-matrix least-squares procedures on $F^{2}$ using SHELXL 2018/3. ${ }^{14}$ All non-hydrogen atoms were refined with anisotropic displacement parameters. All hydrogen atoms were placed in positions of optimized geometry, their isotropic displacement parameters were tied to those of the corresponding carrier atoms by a factor of either 1.2 or 1.5 . Olex 2 was used to prepare material for publication. ${ }^{15}$

Caution! Natural uranium is a radioactive $\alpha$-emitter; and thus, direct and prolonged exposure and inhalation must be prevented. Manipulations should be carried out with care in monitored fume hoods or in inert atmosphere gloveboxes in radiation-controlled laboratories equipped with appropriate radiation counting instruments.

\section{Synthetic and Spectroscopic Details}<smiles>Cc1ccc(O)c([Al])c1</smiles>

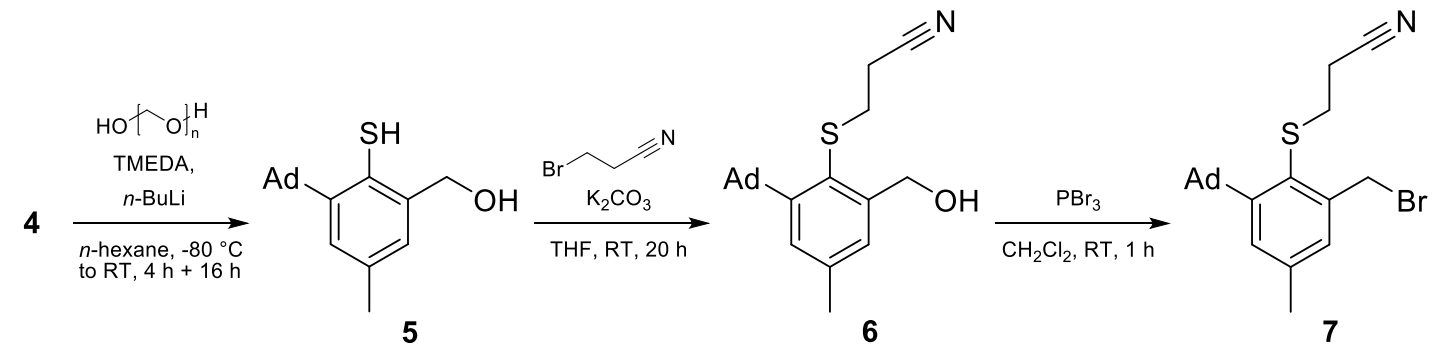

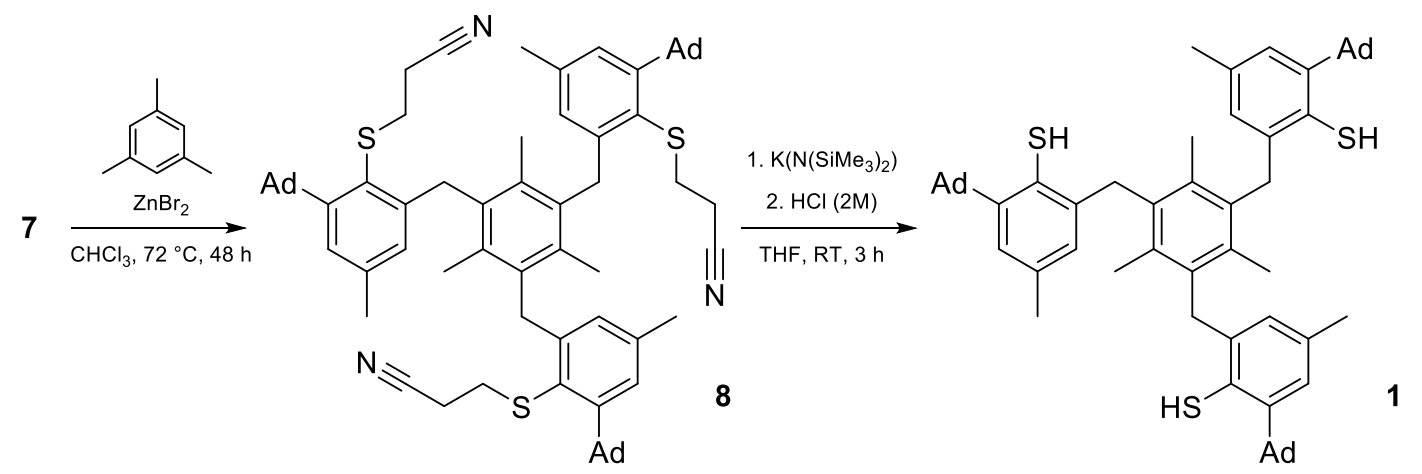

Scheme S1: Overview of the syntheses towards compound mes $\left({ }^{\mathrm{Me}, \mathrm{Ad}} \mathrm{ArSH}\right)_{3}(\mathbf{1})$. 
Synthesis of $O$-(2-adamantyl-4-methylphenyl) dimethylcarbamothioate (2): A flame-dried Schlenk flask under Argon was charged with 2-adamantyl-4-methylphenol (18.17 g, $75.0 \mathrm{mmol})$. The reagent was dissolved in dry NMP $(80 \mathrm{~mL})$ under gentle heating. The solution was then cooled down to $0{ }^{\circ} \mathrm{C}$, and $\mathrm{NaH}(2.15 \mathrm{~g}, 89.6 \mathrm{mmol})$ was added carefully in small portions while stirring. The mixture was stirred at RT for $30 \mathrm{~min}$, cooled again to $0{ }^{\circ} \mathrm{C}$, and a solution of dimethylthiocarbamoyl chloride (12.05 g, $97.5 \mathrm{mmol})$ in NMP $(15 \mathrm{~mL})$ was added dropwise. The mixture was stirred at $90{ }^{\circ} \mathrm{C}$ for $1 \mathrm{~h}$. After cooling down to RT, $\mathrm{H}_{2} \mathrm{O}(200 \mathrm{~mL})$ was added dropwise. $\mathrm{CH}_{2} \mathrm{Cl}_{2}(100 \mathrm{~mL})$ was added, and the mixture was stirred until all solid had dissolved. After separation of the organic phase, the aqueous phase was extracted with $\mathrm{CH}_{2} \mathrm{Cl}_{2}(3 \times 100 \mathrm{~mL})$. The united organics were washed with $\mathrm{H}_{2} \mathrm{O}$ (4x $100 \mathrm{~mL}$ ), dried with anhydrous $\mathrm{MgSO}_{4}$, filtered and the solvent was evaporated to dryness, leaving a brownish residue. The crude product was purified by recrystallization from hot methanol at $-30{ }^{\circ} \mathrm{C}$, giving $\mathbf{2}$ as an off-white solid.

Yield: $21.1 \mathrm{~g}, 64.0 \mathrm{mmol}(84 \%)$.

Analytical data for 2: ${ }^{1} \mathbf{H}$ NMR (400 MHz, chloroform- $\left.d_{3}, 20{ }^{\circ} \mathrm{C} \delta / \mathrm{ppm}\right): 7.16(\mathrm{~s}, 1 \mathrm{H} ; \mathrm{Ar}-H), 7.03$ (d, $J=8 \mathrm{~Hz}, 1 \mathrm{H} ; \mathrm{Ar}-H), 6.90$ (d, J=8 Hz, $1 \mathrm{H} ; \mathrm{Ar}-H), 3.51$ (s, 3H; N-CH3), 3.43 (s, 3H; N-CH3), 2.35 (s, 3H; $\mathrm{Ar}-\mathrm{CH}_{3}$ ), 2.09 (s, 3H; $\mathrm{Ad} \mathrm{CH}$ ), 2.04 (s, 6H; $\mathrm{Ad} \mathrm{CH}_{2}$ ), 1.80-1.72 (m, 6H; $\mathrm{Ad} \mathrm{CH}_{2}$ ).

${ }^{13} \mathrm{C}$ NMR (100.62 MHz, chloroform- $\left.d_{3}, 20^{\circ} \mathrm{C} \delta / \mathrm{ppm}\right): 188.17,150.50,140.62,135.08,127.66,126.73$, $125.24,43.37,41.50,38.84,36.93,28.89,21.29$.

CHNS Elemental analysis calculated for $\mathrm{C}_{20} \mathrm{H}_{27} \mathrm{NOS}$ : C 72.90\%; H 8.26\%; N 4.25\%; S 9.73\%. Found: C 73.05\%; H 8.05\%; N 4.42\%; S 9.60\%.

IR $\left(\mathrm{cm}^{-1}\right)$ : 2906 (s), 2848 (s), 1534 (s), 1488 (s), 1449 (m), 1406 (w), 1386 (s), 1343 (w), 1314 (w), 1277 (s), 1240 (m), 1200 (s), 1183 (s), 1163 (s), $1126(\mathrm{~s}), 1114(\mathrm{~s}), 1046(\mathrm{~m}), 977$ (w), 937 (w), 914 (w), $871(\mathrm{w}), 814(\mathrm{~s}), 789(\mathrm{~m}), 763$ (w), $743(\mathrm{~m}), 669(\mathrm{~m}), 629(\mathrm{~m}), 514(\mathrm{~m}), 471(\mathrm{~m}), 449(\mathrm{~m}), 406(\mathrm{w})$.

Synthesis of $S$-(2-adamantyl-4-methylphenyl) dimethylcarbamothioate (3): Under an atmosphere of argon, elemental analysis pure and dry $O$-(2-adamantyl-4-methylphenyl) dimethylcarbamothioate (2; $12.0 \mathrm{~g}, 36.4 \mathrm{mmol}$ ) was distributed between 3 thick-walled, $15 \mathrm{~mL}$ pressure tubes equipped with Teflon ${ }^{\circledR}$ screw caps. The sealed tubes were placed in a sand bath (pre-heated at $400{ }^{\circ} \mathrm{C}$ ) and were heated at $300{ }^{\circ} \mathrm{C}$ for $18 \mathrm{~h}$. After cooling down to RT, the resinous dark mass was separated from the reaction vessels by melting it with a heat gun. Purification of the crude product by column chromatography $\left(\mathrm{SiO}_{2}\right.$, eluent: $\mathrm{CH}_{2} \mathrm{Cl}_{2}$ ) followed by recrystallization from ethyl acetate at $-30{ }^{\circ} \mathrm{C}$ yielded $\mathbf{3}$ as a white solid.

Rearrangement conversion rate determined by ${ }^{1} \mathrm{H}$ NMR spectroscopy: $>90 \%$. 
Analytical data for 3: ${ }^{1} \mathbf{H}$ NMR $\left(400 \mathrm{MHz}\right.$, chloroform- $\left.d_{3}, 20{ }^{\circ} \mathrm{C} \delta / \mathrm{ppm}\right): 7.33$ (d, $\left.J=8 \mathrm{~Hz}, 1 \mathrm{H} ; \mathrm{Ar}-H\right)$, 7.22 (s, $1 \mathrm{H} ; \mathrm{Ar}-H), 7.05$ (d, J=7.7 Hz, 1H; $\mathrm{Ar}-H), 3.11$ (m, 6H; N-CH3), 2.36 (s, 3H; $\mathrm{Ar}-\mathrm{CH}_{3}$ ), 2.21 (s, 6H; $\mathrm{Ad} \mathrm{CH}_{2}$ ), 2.11 (s, 3H; $\left.\mathrm{Ad} \mathrm{CH}\right), 1.78$ (m, 6H; $\mathrm{Ad} \mathrm{CH}_{2}$ ).

${ }^{13} \mathrm{C}$ NMR (100.62 MHz, chloroform- $\left.d_{3}, 20{ }^{\circ} \mathrm{C} \delta / \mathrm{ppm}\right): 167.58,152.23,141.46,139.37,127.87,127.06$, $123.71,41.32,38.15,36.95,36.75,29.16,21.62$.

CHNS Elemental analysis calculated for $\mathrm{C}_{20} \mathrm{H}_{27} \mathrm{NOS}$ : C 72.90\%; $\mathrm{H} 8.26 \%$; N 4.25\%; S 9.73\%. Found: C 72.66\%; H 8.27\%; N 4.64\%; S 9.62\%.

IR $\left(\mathrm{cm}^{-1}\right)$ : 2912 (s), 2851 (s), 1658 (s), 1596 (m), 1471 (m), 1445 (m), 1404 (m), 1354 (s), 1315 (m), 1259 (m), 1232 (w), 1186 (w), 1091 (s), 1066 (m), 1040 (m), 1029 (m), 979 (w), 913 (s), 869 (m), $816(\mathrm{~s}), 734(\mathrm{~m}), 689(\mathrm{~s}), 654$ (s), $482(\mathrm{~m}), 446(\mathrm{~m})$.

Synthesis of 2-adamantyl-4-methylbenzenethiol (4): A flame-dried Schlenk flask under argon was charged with a solution of crude S-(2-adamantyl-4-methylphenyl) dimethylcarbamothioate $(3 ; 10.7 \mathrm{~g}$, $32.5 \mathrm{mmol})$ in anhydrous THF $(100 \mathrm{~mL})$. The solution was cooled down to $0{ }^{\circ} \mathrm{C}$, and $\mathrm{LiAlH}_{4}(2.4 \mathrm{M}$ in THF; $27.1 \mathrm{~mL}, 65.0 \mathrm{mmol}$ ) was added carefully. The resulting mixture was heated to $70{ }^{\circ} \mathrm{C}$ for $4 \mathrm{~h}$. After cooling again down to $0{ }^{\circ} \mathrm{C}$, the reaction was carefully quenched by dropwise addition of $\mathrm{MeOH}$ $(15 \mathrm{~mL})$, followed by acidification with aqueous $\mathrm{H}_{2} \mathrm{SO}_{4}(5 \% \mathrm{v} / \mathrm{v}, 100 \mathrm{~mL})$. Brine $(40 \mathrm{~mL})$ was added, and the mixture was extracted with $\mathrm{Et}_{2} \mathrm{O}(4 \mathrm{x} 80 \mathrm{~mL})$. The united organics were dried with anhydrous $\mathrm{MgSO}_{4}$, filtered and the solvent was evaporated to dryness. The crude product was purified by plug filtration ( $\mathrm{SiO}_{2}$, eluent: $n$-hexane), giving 4 as an off-white solid.

Yield: $6.38 \mathrm{~g}, 24.7 \mathrm{mmol}(76 \%)$.

Analytical data for 4: ${ }^{1} \mathbf{H}$ NMR (400 MHz, chloroform- $\left.d_{3}, 20{ }^{\circ} \mathrm{C} \delta / \mathrm{ppm}\right)$ : $7.16(\mathrm{~s}, 1 \mathrm{H}, \mathrm{Ar}-H), 7.12(\mathrm{~d}$, $J=7.7 \mathrm{~Hz}, 1 \mathrm{H} ; \mathrm{Ar}-H$ ), 6.87 (d, J=7.7 Hz, 1H; Ar-H), 3.60 (s, 1H; Ar-SH), 2.32 (s, 3H; Ar-CH3), 2.24 (m, 6H; Ad $\mathrm{CH}_{2}$ ), 2.16 (s, 3H; Ad $\left.\mathrm{CH}\right), 1.82$ (m, 6H; $\mathrm{Ad} \mathrm{CH}_{2}$ ).

${ }^{13} \mathbf{C}$ NMR (100.62 MHz, chloroform- $\left.d_{3}, 20{ }^{\circ} \mathrm{C} \delta / \mathrm{ppm}\right): 147.37,135.27,133.75,127.90,126.93,125.99$, $40.09,37.69,36.75,29.05,21.12$.

CHS Elemental analysis calculated for $\mathrm{C}_{17} \mathrm{H}_{22} \mathrm{~S}$ : C 79.01\%; H 8.58\%; S 12.41\%. Found: C 79.27\%; H $8.51 \%$; S $12.06 \%$.

IR $\left(\mathrm{cm}^{-1}\right)$ : 2897 (s), 2848 (s), 1471 (s), 1446 (s), 1394 (w), 1366 (w), 1357 (w), 1343 (m), 1314 (m), 1286 (w), 1260 (m), 1246 (w), 1189 (w), 1143 (w), 1100 (m), 1071 (m), 1029 (m), 974 (w), 946 (w), $909(\mathrm{w}), 874(\mathrm{w}), 811(\mathrm{~s}), 691(\mathrm{w}), 646(\mathrm{w}), 629(\mathrm{w}), 580(\mathrm{w}), 469(\mathrm{~s}), 449(\mathrm{~m}), 409(\mathrm{w})$.

Synthesis of (3-adamantyl-2-mercapto-5-methylphenyl)methanol (5): A flame-dried Schlenk flask under argon was charged with a solution of TMEDA $(9.1 \mathrm{~mL}, 7.0 \mathrm{~g}, 60 \mathrm{mmol})$ in dry $n$-hexane $(10 \mathrm{~mL})$ 
and cooled down to $-80^{\circ} \mathrm{C}$. $n$-Buthyllithium ( $2.5 \mathrm{M}$ in $n$-hexane; $\left.24 \mathrm{~mL}, 60 \mathrm{mmol}\right)$ was added slowly, and the solution was stirred for $10 \mathrm{~min}$. A solution of 2-adamantyl-4-methylbenzenethiol $(4 ; 7.05 \mathrm{~g}$, $27.3 \mathrm{mmol})$ in $n$-hexane $(100 \mathrm{~mL})$ was added dropwise. The dark yellow solution was stirred at RT for $4 \mathrm{~h}$. The reaction was cooled down again to $-80^{\circ} \mathrm{C}$, and paraformaldehyde $(2.05 \mathrm{~g}, 68.3 \mathrm{mmol})$ was added. The reaction solution was stirred for $16 \mathrm{~h}$ at RT. After cooling down to $0{ }^{\circ} \mathrm{C}$, the mixture was quenched by slow addition of $\mathrm{H}_{2} \mathrm{O}(100 \mathrm{~mL})$, and the $\mathrm{pH}$ value was carefully adjusted to $\mathrm{pH}=6$ by addition of aqueous $\mathrm{HCl}(50 \% \mathrm{v} / \mathrm{v})$. The resulting mixture was extracted with $\mathrm{Et}_{2} \mathrm{O}(4 \times 100 \mathrm{~mL})$. The united organics were dried with anhydrous $\mathrm{MgSO}_{4}$, filtered and the solvent was evaporated to dryness. The crude product was purified by column chromatography $\left(\mathrm{SiO}_{2}\right.$, eluent: $\mathrm{CH}_{2} \mathrm{Cl}_{2}: \mathrm{MeOH}$, gradient: $100 \% \mathrm{CH}_{2} \mathrm{Cl}_{2}$ to $25: 1$ ), giving 5 as an off-white solid.

Yield: $4.41 \mathrm{~g}, 15.3 \mathrm{mmol}(56 \%)$.

Analytical data for 5: ${ }^{1} \mathbf{H}$ NMR (400 MHz, chloroform- $\left.d_{3}, 20^{\circ} \mathrm{C} \delta / \mathrm{ppm}\right): 7.16(\mathrm{~s}, 1 \mathrm{H} ; \operatorname{Ar}-H), 7.05$ (s, $1 \mathrm{H} ; \mathrm{Ar}-\mathrm{H}), 4.78$ (s, 2H; benzylic $\mathrm{CH}_{2}$ ), 4.31 (s, $\left.1 \mathrm{H} ; \mathrm{Ar}-\mathrm{SH}\right), 2.32$ (s, 3H; $\left.\mathrm{Ar}-\mathrm{CH}_{3}\right), 2.29$ (m, 6H; Ad $\mathrm{CH}_{2}$ ), 2.14 (s, 3H; Ad $\mathrm{CH}$ ), 1.92 (s, 1H; benzylic $\left.\mathrm{OH}\right), 1.85-1.76$ (m, 6H; $\mathrm{Ad} \mathrm{CH}_{2}$ ).

${ }^{13} \mathrm{C}$ NMR (100.62 MHz, chloroform- $\left.d_{3}, 20{ }^{\circ} \mathrm{C} \delta / \mathrm{ppm}\right): 149.93,140.52,135.59,128.16,127.92,126.22$, $66.43,40.50,38.43,36.76,29.20,21.14$.

CHS Elemental analysis calculated for $\mathrm{C}_{18} \mathrm{H}_{24} \mathrm{OS}$ : C 74.95\%; H 8.39\%; S 11.11\%. Found: C 75.26\%; H $8.18 \%$; S $10.76 \%$.

IR ( $\left.\mathrm{cm}^{-1}\right)$ : 3406 (bm), 2900 (s), 2851 (s), 2540 (w), 1597 (m), 1445 (s), 1426 (s), 1391 (m), 1369 (m), 1343 (m), 1317 (m), 1277 (m), 1246 (w), 1180 (m), 1169 (m), 1089 (s), 1054 (s), 1000 (s), 974 (s), 943 (m), 886 (m), 857 (s), 820 (m), 791 (m), 757 (m), 734 (w), 674 (w), 649 (m), 574 (w), 543 (w), $509(\mathrm{w}), 431(\mathrm{w})$.

Synthesis of 3-((2-adamantyl-6-(hydroxymethyl)-4-methylphenyl)thio)propanenitrile (6): A flame-dried Schlenk flask under argon was charged with a solution of (3-adamantyl-2-mercapto-5methylphenyl)methanol (5; $2.56 \mathrm{~g}, 8.88 \mathrm{mmol})$ in dry THF $(60 \mathrm{~mL})$. Solid $\mathrm{K}_{2} \mathrm{CO}_{3}(3.06 \mathrm{~g}, 22.1 \mathrm{mmol})$ was added, and the mixture was stirred for $15 \mathrm{~min}$. A solution of 3-bromopropionitrile $(2.2 \mathrm{~mL}, 3.6 \mathrm{~g}$, $27 \mathrm{mmol})$ in THF (10 mL) was added, and the solution was stirred at RT for $20 \mathrm{~h}$. After cooling down to $0{ }^{\circ} \mathrm{C}$, the reaction was quenched by addition of aqueous $\mathrm{HCl}(5 \% \mathrm{v} / \mathrm{v} ; 75 \mathrm{~mL})$, and the mixture was extracted with $\mathrm{CH}_{2} \mathrm{Cl}_{2}(4 \mathrm{x} 60 \mathrm{~mL})$. The united organics were dried with anhydrous $\mathrm{MgSO}_{4}$, filtered and the solvent was evaporated to dryness. The crude product was purified by column chromatography ( $\mathrm{SiO}_{2}$, eluent: $n$-hexane:EtOAc, gradient: 2:1 to 1:1), giving 6 as a white solid.

Yield: $2.46 \mathrm{~g}, 7.20 \mathrm{mmol}(81 \%)$. 
Analytical data for 6: ${ }^{1} \mathbf{H}$ NMR $\left(400 \mathrm{MHz}\right.$, chloroform- $\left.d_{3}, 20{ }^{\circ} \mathrm{C} \delta / \mathrm{ppm}\right): 7.24(\mathrm{~s}, 1 \mathrm{H} ; \mathrm{Ar}-H), 7.19$ (s, $1 \mathrm{H} ; \mathrm{Ar}-H), 4.94\left(\mathrm{~s}, 2 \mathrm{H}\right.$; benzylic $\left.\mathrm{CH}_{2}\right), 2.97\left(\mathrm{t}, J=8 \mathrm{~Hz}, 2 \mathrm{H}\right.$; propionitrilic $\left.\mathrm{CH}_{2}\right), 2.64(\mathrm{t}, J=8 \mathrm{~Hz}, 2 \mathrm{H}$; propionitrilic $\mathrm{CH}_{2}$ ), 2.36 (s, 3H; $\left.\mathrm{Ar}-\mathrm{CH}_{3}\right), 2.31$ (s, $1 \mathrm{H}$; benzylic $\mathrm{OH}$ ), 2.28 (m, 6H; $\mathrm{Ad} \mathrm{CH}_{2}$ ), 2.14 (s, 3H; Ad $\mathrm{CH}), 1.85-1.76\left(\mathrm{~m}, 6 \mathrm{H} ; \mathrm{Ad} \mathrm{CH}_{2}\right.$ ).

${ }^{13} \mathrm{C}$ NMR (100.62 MHz, chloroform- $\left.d_{3}, 20{ }^{\circ} \mathrm{C} \delta / \mathrm{ppm}\right): 153.96,146.70,139.36,128.21,127.93,127.61$, $118.00,64.52,41.83,39.22,36.60,33.75,29.28,21.51,17.25$.

CHNS Elemental analysis calculated for $\mathrm{C}_{21} \mathrm{H}_{27} \mathrm{NOS}$ : C 73.86\%; H 7.97\%; N 4.10\%; S 9.39\%. Found: C 73.63\%; H 7.89\%; N 4.04\%; S 9.06\%.

IR $\left(\mathrm{cm}^{-1}\right)$ : 3383 (m), 3313 (m), 2893 (s), 2846 (s), 2257 (w), 1597 (m), 1426 (m), 1379 (m), 1368 (m), 1357 (m), 1341 (m), 1311 (m), 1240 (w), 1184 (w), 1166 (w), 1077 (m), 1049 (s), 1019 (s), 966 (m), $897(\mathrm{~m}), 875(\mathrm{w}), 858(\mathrm{~s}), 818(\mathrm{~m}), 788(\mathrm{w}), 765(\mathrm{~m}), 721(\mathrm{~m}), 648(\mathrm{~m}), 575(\mathrm{~m}), 534(\mathrm{~m}), 437(\mathrm{w})$, $419(\mathrm{w})$.

Synthesis of 3-((2-adamantyl-6-(bromomethyl)-4-methylphenyl)thio)propanenitrile (7): A flamedried Schlenk flask under argon was charged with a solution of 3-((2-adamantyl-6-(hydroxymethyl)-4methylphenyl)thio)propanenitrile $(6 ; 2.64 \mathrm{~g}, 7.73 \mathrm{mmol})$ in dry $\mathrm{CH}_{2} \mathrm{Cl}_{2}(50 \mathrm{~mL})$. The mixture was cooled down to $0{ }^{\circ} \mathrm{C}$, and a solution of $\mathrm{PBr}_{3}(0.95 \mathrm{~mL}, 10.0 \mathrm{mmol})$ in $\mathrm{CH}_{2} \mathrm{Cl}_{2}(10 \mathrm{~mL})$ was added carefully over $10 \mathrm{~min}$. The resulting solution was stirred at RT for $1 \mathrm{~h}$ and then quenched by careful addition of $\mathrm{H}_{2} \mathrm{O}(50 \mathrm{~mL})$. The mixture was extracted with $\mathrm{CH}_{2} \mathrm{Cl}_{2}(4 \times 60 \mathrm{~mL})$. The united organics were dried with anhydrous $\mathrm{MgSO}_{4}$, filtered and the solvent was evaporated to dryness. The crude product was purified by plug filtration $\left(\mathrm{SiO}_{2}\right.$, eluent: $\left.\mathrm{CH}_{2} \mathrm{Cl}_{2}\right)$, giving 7 as a white solid.

Yield: $2.31 \mathrm{~g}, 5.71 \mathrm{mmol}(74 \%)$.

Analytical data for 7: ${ }^{1} \mathbf{H}$ NMR (400 MHz, chloroform- $\left.d_{3}, 20{ }^{\circ} \mathrm{C} \delta / \mathrm{ppm}\right): 7.30$ (s, $\left.1 \mathrm{H} ; \mathrm{Ar}-H\right), 7.20$ (s, 1H; $\operatorname{Ar}-H$ ), 4.98 (s, 2H; benzylic $\mathrm{CH}_{2}$ ), 3.09 (t, $J=7.3 \mathrm{~Hz}, 2 \mathrm{H}$; propionitrilic $\mathrm{CH}_{2}$ ), 2.68 (t, $J=7.4 \mathrm{~Hz}$, 2H; propionitrilic $\mathrm{CH}_{2}$ ), 2.35 (s, 3H; $\mathrm{Ar}-\mathrm{CH}_{3}$ ), 2.27 (m, 6H; $\mathrm{Ad} \mathrm{CH}_{2}$ ), 2.14 (s, 3H; $\mathrm{Ad} \mathrm{CH}$ ), 1.84-1.76 (m, 6H; $\mathrm{Ad} \mathrm{CH}_{2}$ ).

${ }^{13} \mathrm{C}$ NMR (100.62 MHz, chloroform- $\left.d_{3}, 20{ }^{\circ} \mathrm{C} \delta / \mathrm{ppm}\right): 154.40,144.03,139.62,130.67,129.15,128.90$, $117.88,41.83,39.48,36.60,33.72,33.37,29.29,21.40,17.26$.

CHNS Elemental analysis calculated for $\mathrm{C}_{21} \mathrm{H}_{26} \mathrm{BrNS}$ : C 62.37\%; H 6.48\%; N 3.46\%; S 7.93\%. Found: C $62.44 \%$; H $6.42 \%$; N 3.47\%; S 7.71\%.

IR ( $\left.\mathrm{cm}^{-1}\right)$ : 2904 (s), 2843 (s), 2246 (w), 1599 (s), 1472 (w), 1449 (m), 1439 (m), 1428 (s), 1416 (s), 1378 (w), 1365 (w), 1340 (m), 1314 (w), 1281 (s), 1221 (m), 1207 (m), 1181 (w), 1149 (w), 1118 (w), 1102 (m), 1075 (m), 1044 (m), 1024 (w), 979 (w), 960 (m), 912 (m), 854 (m), 819 (m), 787 (w), 755 (w), $748(\mathrm{~m}), 679(\mathrm{w}), 654(\mathrm{~s}), 644(\mathrm{~m}), 633(\mathrm{w}), 584(\mathrm{~s}), 566(\mathrm{~m}), 511(\mathrm{~s}), 416(\mathrm{w})$. 
Synthesis of mes( $\left({ }^{\mathrm{Me}, \mathrm{Ad}} \mathrm{ArS}\left(\mathrm{CH}_{2}\right)_{2} \mathrm{CN}\right)_{3}(\mathbf{8})$ : A flame-dried Schlenk flask under argon was charged with a solution of 3-((2-adamantyl-6-(bromomethyl)-4-methylphenyl)thio)propanenitrile $\quad(7 ; 1.79 \mathrm{~g}$, $4.43 \mathrm{mmol})$ in dry $\mathrm{CHCl}_{3}(70 \mathrm{~mL})$. Mesitylene $(153 \mu \mathrm{L}, 1.11 \mathrm{mmol})$ was added, followed by dry $\mathrm{ZnBr}_{2}$ $(1.25 \mathrm{~g}, 5.55 \mathrm{mmol})$. The mixture was refluxed at $72{ }^{\circ} \mathrm{C}$ for $48 \mathrm{~h}$. The milky reaction solution was then cooled down to RT and quenched by addition of a saturated $\mathrm{NH}_{4} \mathrm{Cl}$ solution $(50 \mathrm{~mL})$. The mixture was extracted with $\mathrm{CH}_{2} \mathrm{Cl}_{2}(4 \mathrm{x} 50 \mathrm{~mL})$. The united organics were dried with anhydrous $\mathrm{MgSO}_{4}$, filtered and the solvent was evaporated to dryness. The crude product was purified by column chromatography $\left(\mathrm{SiO}_{2}\right.$, eluent: $\left.\mathrm{CH}_{2} \mathrm{Cl}_{2}\right)$. The residue was dried in vacuo for $16 \mathrm{~h}$ at $85^{\circ} \mathrm{C}$, brought into a Glovebox, dissolved in benzene, and lyophilized until solvent free, giving $\mathbf{8}$ as a white powder.

Yield: $824 \mathrm{mg}, 0.755 \mathrm{mmol}(68 \%)$.

Analytical data for 8: ${ }^{1} \mathbf{H}$ NMR (400 MHz, chloroform- $\left.d_{3}, 20{ }^{\circ} \mathrm{C} \delta / \mathrm{ppm}\right)$ : 7.11 (s, 3H; $\mathrm{Ar}-H$ ), 6.88 (s, $3 \mathrm{H}$; $\mathrm{Ar}-\mathrm{H}$ ), 4.41 (s, br, $6 \mathrm{H}$; benzylic $\mathrm{CH}_{2}$ ), 2.42 (t, $J=7.3 \mathrm{~Hz}, 6 \mathrm{H}$; propionitrilic $\mathrm{CH}_{2}$ ), 2.36 (s, $18 \mathrm{H}$; Ad $\mathrm{CH}_{2}$ ), 2.22 (s, 9H; $\mathrm{Ar}-\mathrm{CH}_{3}$ ), 2.16 (s, 9H; $\left.\mathrm{Ar}-\mathrm{CH}_{3}\right), 2.14$ (s, 9H; $\left.\mathrm{Ad} \mathrm{CH}\right), 1.91-1.76$ (m, 18H; Ad $\left.\mathrm{CH}_{2}\right), 1.76\left(\mathrm{t}, \mathrm{J}=7.3 \mathrm{~Hz}, 6 \mathrm{H}\right.$; propionitrilic $\mathrm{CH}_{2}$ ).

${ }^{13} \mathrm{C}$ NMR (100.62 MHz, chloroform- $\left.d_{3}, 20{ }^{\circ} \mathrm{C} \delta / \mathrm{ppm}\right): 154.39,147.43,139.07,137.40,135.68,129.94$, $128.93,127.82$, 127.05, 118.20, 42.54, 39.96, 37.42, 36.10, 32.70, 30.23, 22.29, 17.39, 17.23.

CHNS Elemental analysis calculated for $\mathrm{C}_{72} \mathrm{H}_{87} \mathrm{~N}_{3} \mathrm{~S}_{3}$ : C 79.29\%; $\mathrm{H} 8.04 \%$; N 3.85\%; S 8.82\%. Found: C 79.39\%; H 8.18\%; N 3.65\%; S 8.69\%.

IR $\left(\mathrm{cm}^{-1}\right)$ : 2900 (s), 2847 (s), 1596 (m), 1554 (w), 1448 (m), 1418 (m), $1356(\mathrm{w}), 1343$ (m), $1314(\mathrm{~m})$, $1279(\mathrm{w}), 1239$ (w), 1186 (w), 1104 (m), 1067 (w), 1036 (m), 1017 (m), 976 (w), 957 (w), 906 (w), 854 (s), $818(\mathrm{~m}), 739$ (w), 678 (s), 654 (m), 564 (m), 483 (w), 419 (w).

Synthesis of mes( $\left({ }^{\mathrm{Me}, \mathrm{Ad}} \mathrm{ArSH}\right)_{3}$ (1): Inside a glovebox, $\operatorname{mes}\left({ }^{\mathrm{Me}, \mathrm{Ad}} \mathrm{ArS}\left(\mathrm{CH}_{2}\right)_{2} \mathrm{CN}\right)_{3} \quad(\mathbf{8} ; 217 \mathrm{mg}$, $0.199 \mathrm{mmol})$ was dissolved in THF $(20 \mathrm{~mL})$ and loaded into a single neck, $100 \mathrm{~mL}$ Teflon ${ }^{\circledR}$-capped bomb flask equipped with a stir bar. A solution of $\mathrm{K}\left(\mathrm{N}\left(\mathrm{SiMe}_{3}\right)_{2}\right)(160 \mathrm{mg}, 0.800 \mathrm{mmol})$ in $\mathrm{THF}(5 \mathrm{~mL})$ was added dropwise while stirring. The resulting milky yellow solution was stirred at RT for additional $3 \mathrm{~h}$. The mixture was then cooled down to $0^{\circ} \mathrm{C}$ in an ice bath, and carefully acidified with aqueous $\mathrm{HCl}$ ( $2 \mathrm{M}, 1 \mathrm{~mL})$. The formation of an insoluble, yellowish precipitate could be observed. $\mathrm{CH}_{2} \mathrm{Cl}_{2}(15 \mathrm{~mL})$ was added, and the mixture was stirred for a few minutes. After separation of the organic phase, the aqueous layer was extracted with additional $\mathrm{CH}_{2} \mathrm{Cl}_{2}(3 \times 10 \mathrm{~mL})$. The united organics were washed with $\mathrm{H}_{2} \mathrm{O}(20 \mathrm{~mL})$, dried with anhydrous $\mathrm{MgSO}_{4}$, filtered and the solvent was evaporated to dryness, leaving an off-white residue, which was purified by plug filtration $\left(\mathrm{SiO}_{2}\right.$, eluent: $\left.\mathrm{CH}_{2} \mathrm{Cl}_{2}\right)$. The residue was dried in vacuo for $16 \mathrm{~h}$ at $85^{\circ} \mathrm{C}$, brought into a glovebox, dissolved in benzene, and lyophilized until solvent free, giving 1 as a white powder.

Yield: $162 \mathrm{mg}, 0.174 \mathrm{mmol}(87 \%)$. 
Analytical data for 1: ${ }^{1} \mathbf{H}$ NMR $\left(400 \mathrm{MHz}\right.$, benzene- $\left.d_{6}, 20^{\circ} \mathrm{C} \delta / \mathrm{ppm}\right): 7.16(\mathrm{~s}, 3 \mathrm{H} ; \mathrm{Ar}-H), 6.80$ (s, 3H; $\mathrm{Ar}-\mathrm{H}$ ), 3.96 (s, 6H; benzylic $\mathrm{CH}_{2}$ ), 3.15 (s, 3H; $\mathrm{Ar}-\mathrm{SH}$ ), 2.43 (s, $18 \mathrm{H} ; \mathrm{Ad} \mathrm{CH}_{2}$ ), 2.26 (s, 9H; $\mathrm{Ar}-\mathrm{CH}_{3}$ ), 2.10 (s, 9H; Ad $\mathrm{CH}$ ), 2.00 (s, 9H; $\left.\mathrm{Ar}-\mathrm{CH}_{3}\right), 1.85-1.72$ (m, 18H; $\mathrm{Ad} \mathrm{CH}_{2}$ ).

${ }^{13} \mathrm{C}$ NMR $\left(100.62 \mathrm{MHz}\right.$, benzene- $\left.d_{6}, 20{ }^{\circ} \mathrm{C} \delta / \mathrm{ppm}\right): 150.11,141.59,136.28,135.78,128.92,126.91$, $126.67,41.37,39.27,38.20,37.53,30.13,22.23,16.92$.

CHS Elemental analysis calculated for $\mathrm{C}_{63} \mathrm{H}_{78} \mathrm{~S}_{3}$ : C 81.23\%; H 8.44\%; S 10.33\%. Found: C 81.34\%; H $8.28 \%$; S 9.63\%.

IR $\left(\mathrm{cm}^{-1}\right)$ : 2901 (s), 2847 (s), 1598 (m), 1559 (w), 1473 (w), 1447 (m), 1358 (w), 1343 (m), 1314 (m), 1187 (w), 1170 (w), 1104 (m), 1069 (m), 1047 (m), 1019 (m), 977 (m), 879 (w), 854 (s), 817 (m), 724 (m), $676(\mathrm{~s}), 654(\mathrm{w}), 584(\mathrm{~m}), 556(\mathrm{~m}), 531(\mathrm{w}), 469(\mathrm{w}), 431(\mathrm{w}), 411(\mathrm{w})$.

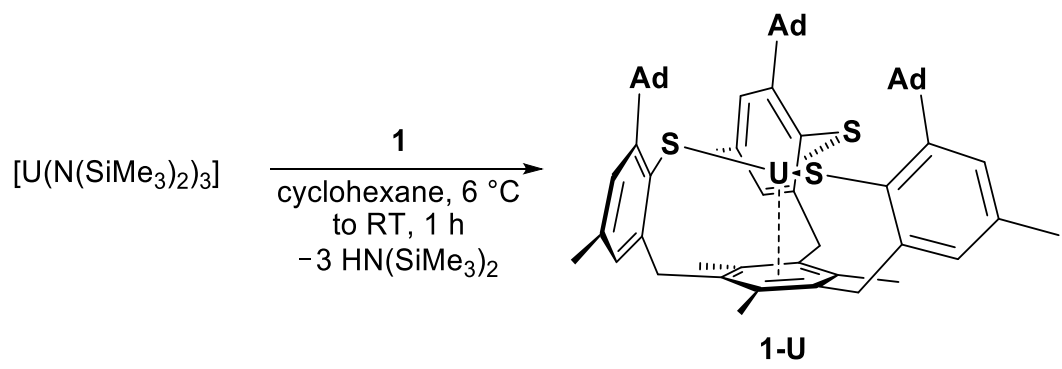

Scheme S2: Synthesis of [ $\left.\mathrm{U}^{\mathrm{III}}\left(\left(\mathrm{SAr}^{\mathrm{Ad}, \mathrm{Me}}\right)_{3} \mathrm{mes}\right)\right](\mathbf{1 - U})$.

Synthesis of [ $\left.\mathbf{U}^{\mathrm{III}}\left(\left(\mathrm{SAr}^{\mathrm{Ad}, \mathrm{Me}}\right)_{3} \mathrm{mes}\right)\right]$ (1-U): Inside a glovebox, a thawing solution of [U(N($\left.\left.\left(\mathrm{SiMe}_{3}\right)_{2}\right)_{3}\right]$ $(131 \mathrm{mg}, 0.182 \mathrm{mmol})$ in cyclohexane $(5 \mathrm{~mL})$ was added dropwise to a thawing mixture of $\operatorname{mes}\left({ }^{\mathrm{Me}, \mathrm{Ad}} \mathrm{ArSH}\right)_{3}(\mathbf{1} ; 113 \mathrm{mg}, 0.121 \mathrm{mmol})$ in cyclohexane $(10 \mathrm{~mL})$. The dark reaction mixture was stirred for $1.5 \mathrm{~h}$ at RT, during which a dark purple solid precipitated. The reaction mixture was filtered through a glass frit, and the solid was washed with cyclohexane $(3 \times 1.5 \mathrm{~mL})$ and $n$-pentane $(3 \times 1.5 \mathrm{~mL})$. The powder was then dissolved in THF $(5 \mathrm{~mL})$, and the volatiles were removed in vacuo, leaving a dark brown residue, which was triturated twice with $n$-pentane $(5 \mathrm{~mL})$. The removal of volatiles in vacuo afforded the title complex 1-U as a purple (lilac) powder.

Yield: $117 \mathrm{mg}, 0.100 \mathrm{mmol}(83 \%)$.

Analytical data for 1-U: ${ }^{1} \mathbf{H}$ NMR (400 MHz, toluene- $\left.d_{8}, 20{ }^{\circ} \mathrm{C} \delta / \mathrm{ppm}\right): 11.71,11.02,8.70,5.99,3.52$, -2.20 (dd, $J=192 \mathrm{~Hz}, 12 \mathrm{~Hz}),-2.40,-3.61,-8.38,-29.24,-30.55$.

${ }^{1}$ H NMR (400 MHz, tetrahydrofuran- $\left.d_{8}, 20{ }^{\circ} \mathrm{C} \delta / \mathrm{ppm}\right): 11.49(\mathrm{~s}, 3 \mathrm{H}), 6.67(\mathrm{~s}, 3 \mathrm{H}), 6.55$ (br s, 18H), $4.41(\mathrm{~s}, 9 \mathrm{H}), 2.01(\mathrm{~s}, 9 \mathrm{H}), 1.60(\mathrm{~s}, 9 \mathrm{H}), 1.36(\mathrm{~s}, 9 \mathrm{H}),-20.87$ (br s, 6H), -34.43 (br s, 9H).

CHS Elemental analysis calculated for $\mathrm{C}_{63} \mathrm{H}_{75} \mathrm{~S}_{3} \mathrm{U}$ : C 64.87\%; H 6.48\%; S 8.25\%. Found: $\mathrm{C} 65.06 \%$; H 6.64\%; S 7.10\%. 
IR ( $\left.\mathrm{cm}^{-1}\right)$ : 3086 (w), 3032 (w), 2901 (s), 2847 (s), 1597 (m), 1443 (s), 1420 (m), 1369 (m), 1342 (m), 1312 (m), 1269 (w), 1246 (w), 1188 (w), 1103 (m), 1034 (s), 980 (m), 910 (w), 887 (w), $856(\mathrm{~m})$, $818(\mathrm{~m}), 791(\mathrm{w}), 737$ (w), $675(\mathrm{~s}), 648(\mathrm{w}), 621(\mathrm{w}), 586(\mathrm{w}), 556(\mathrm{w}), 517(\mathrm{w}), 467$ (w).

${ }^{1} \mathrm{H}$ and ${ }^{13} \mathrm{C}$ NMR Spectroscopy

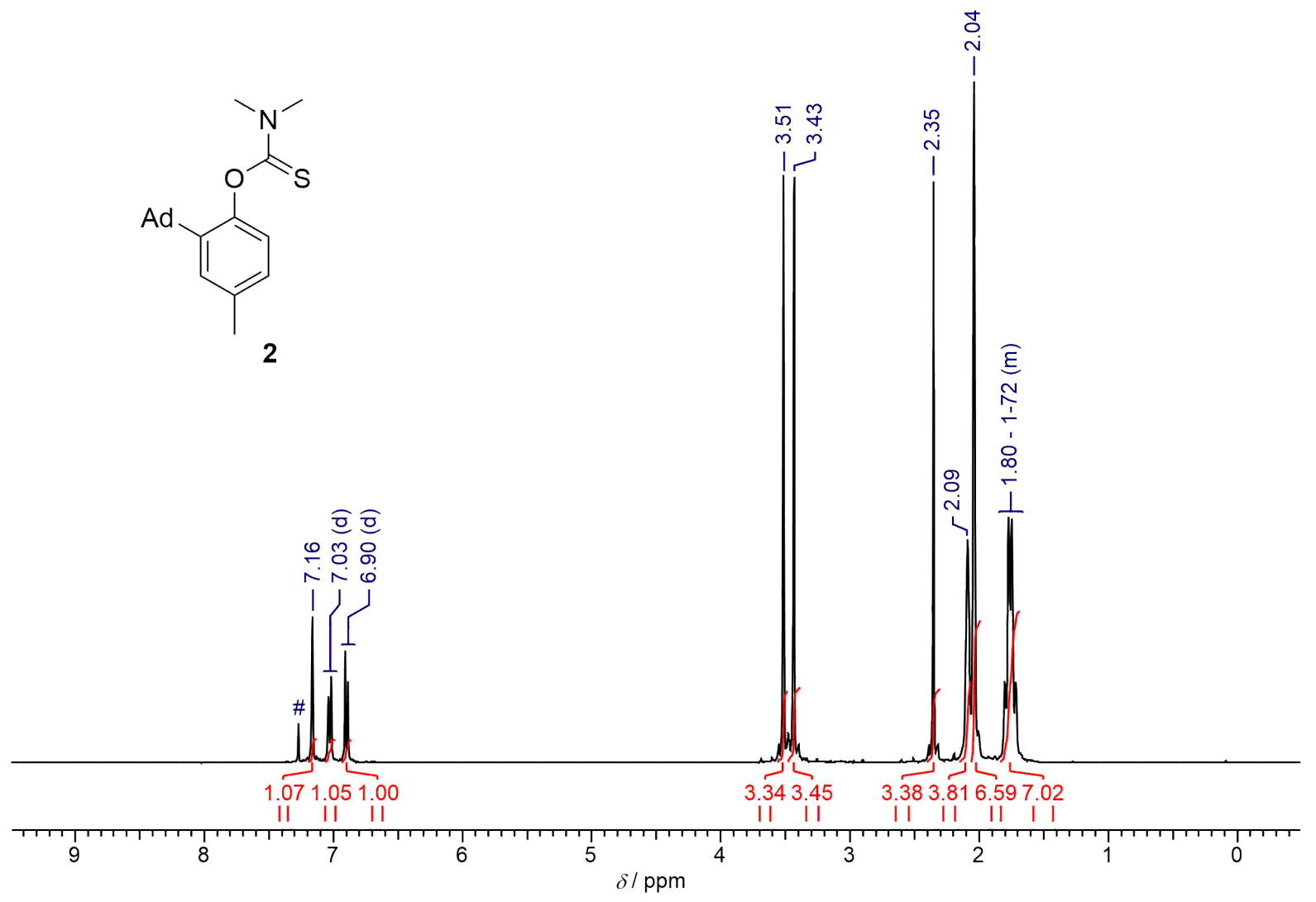

Figure S1: ${ }^{1} \mathrm{H}$ NMR of 2 recorded in chloroform- $d_{3}(\#)$. 


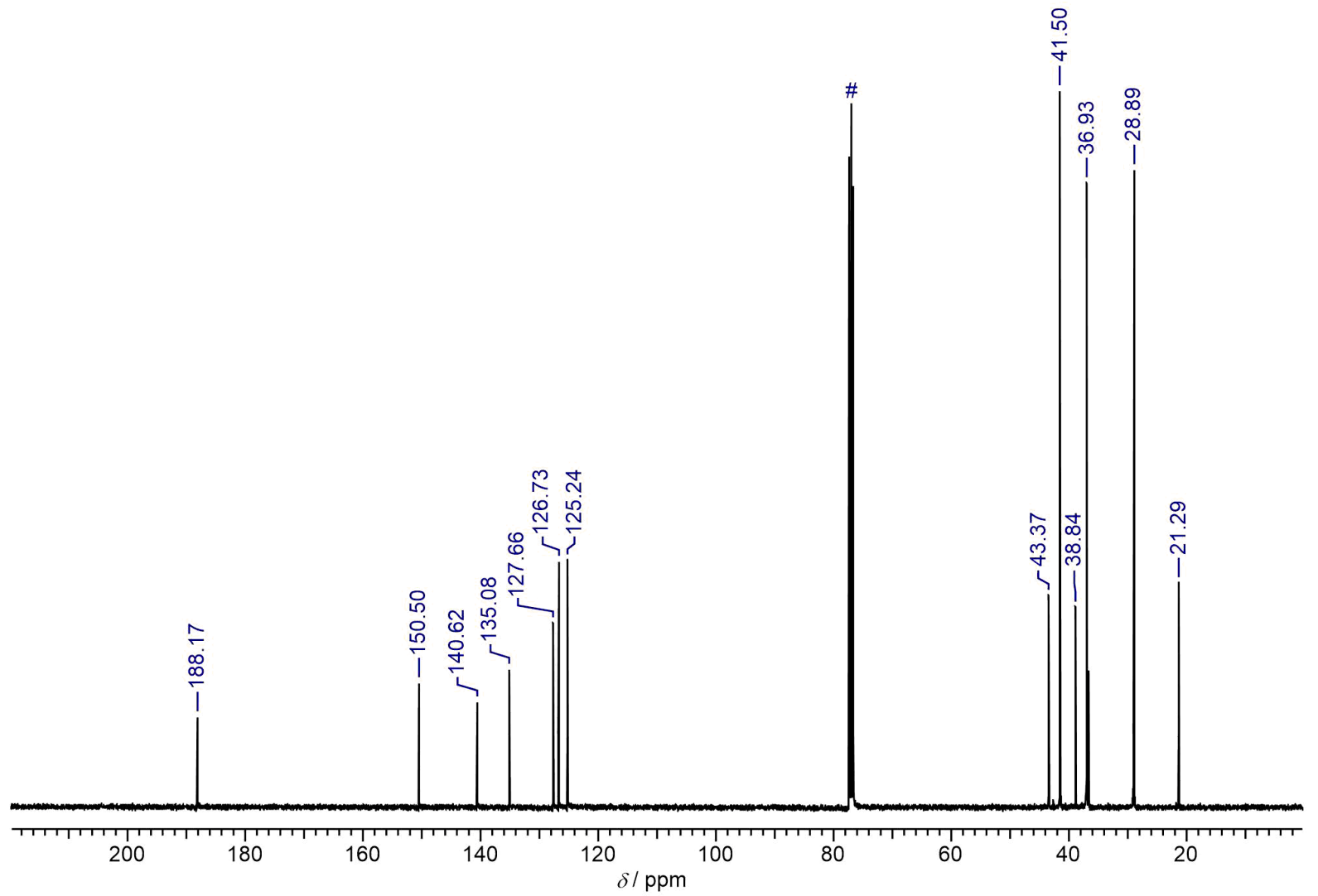

Figure S2: ${ }^{13} \mathrm{C}$ NMR of 2 recorded in chloroform- $d_{3}(\#)$.

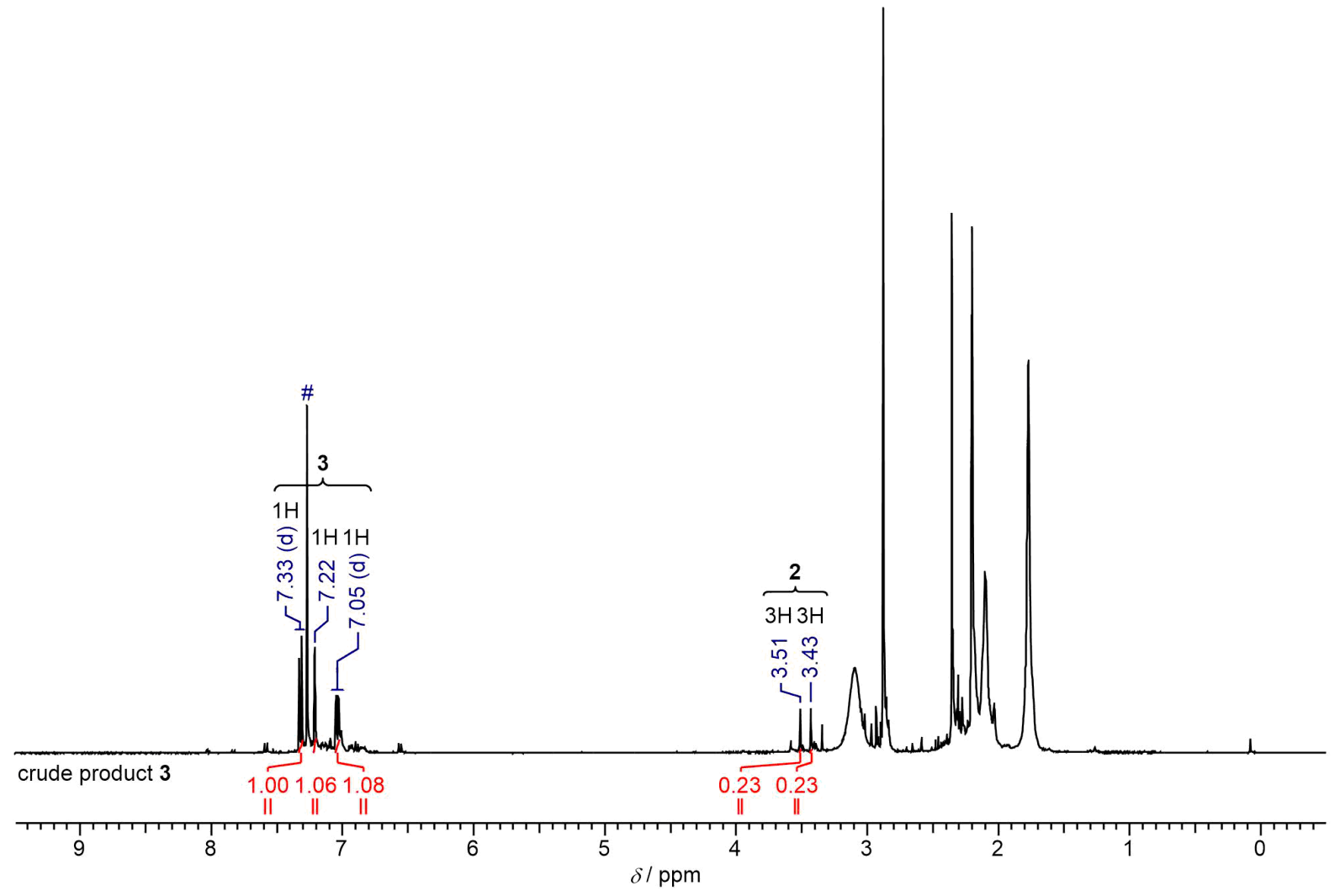

Figure S3: ${ }^{1} \mathrm{H}$ NMR of crude product 3 recorded in chloroform- $d_{3}(\#)$. 


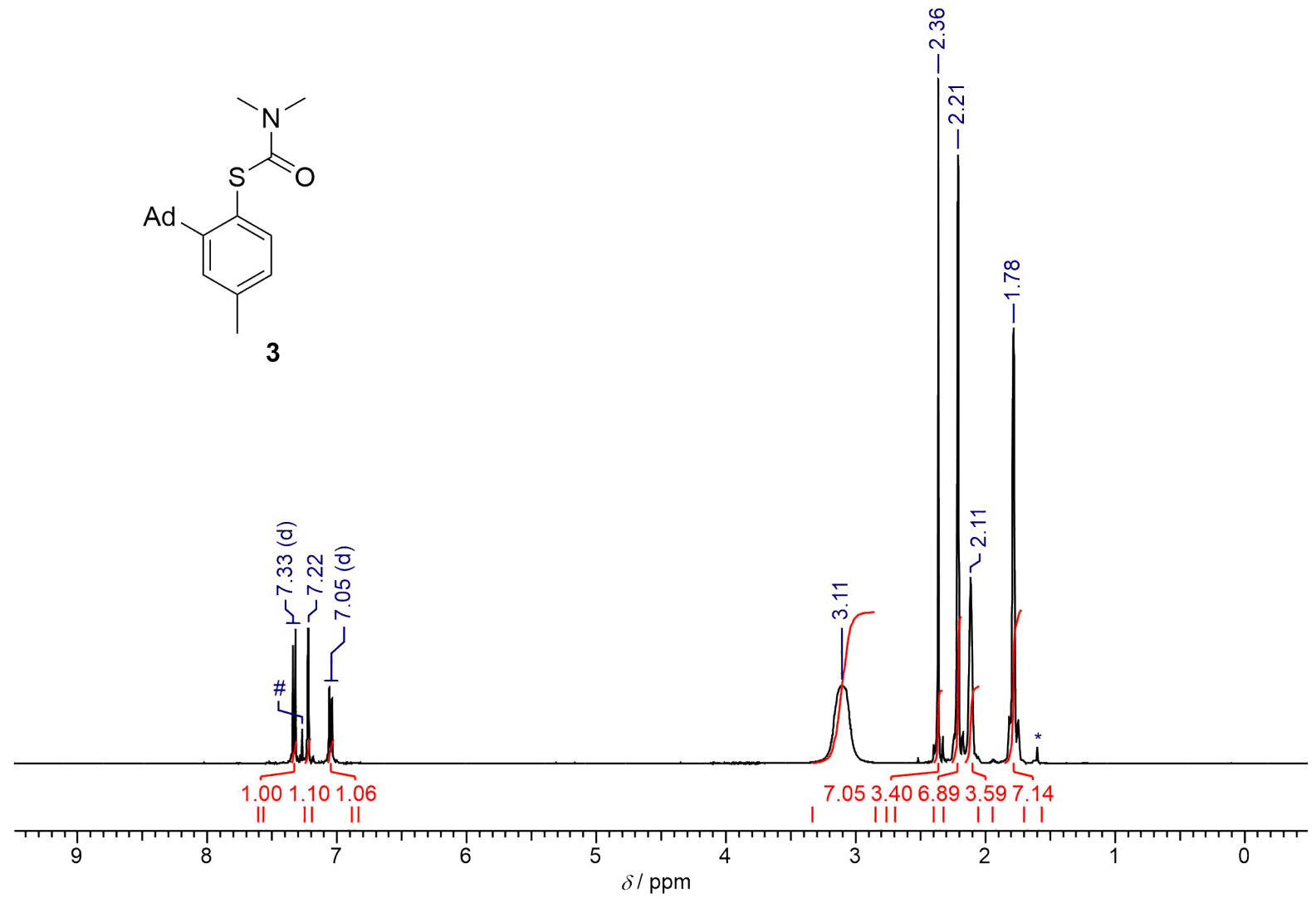

Figure S4: ${ }^{1} \mathrm{H}$ NMR of 3 recorded in chloroform- $d_{3}(\#)$. Residual $\mathrm{H}_{2} \mathrm{O}$ is marked with an asterisk $(*)$.

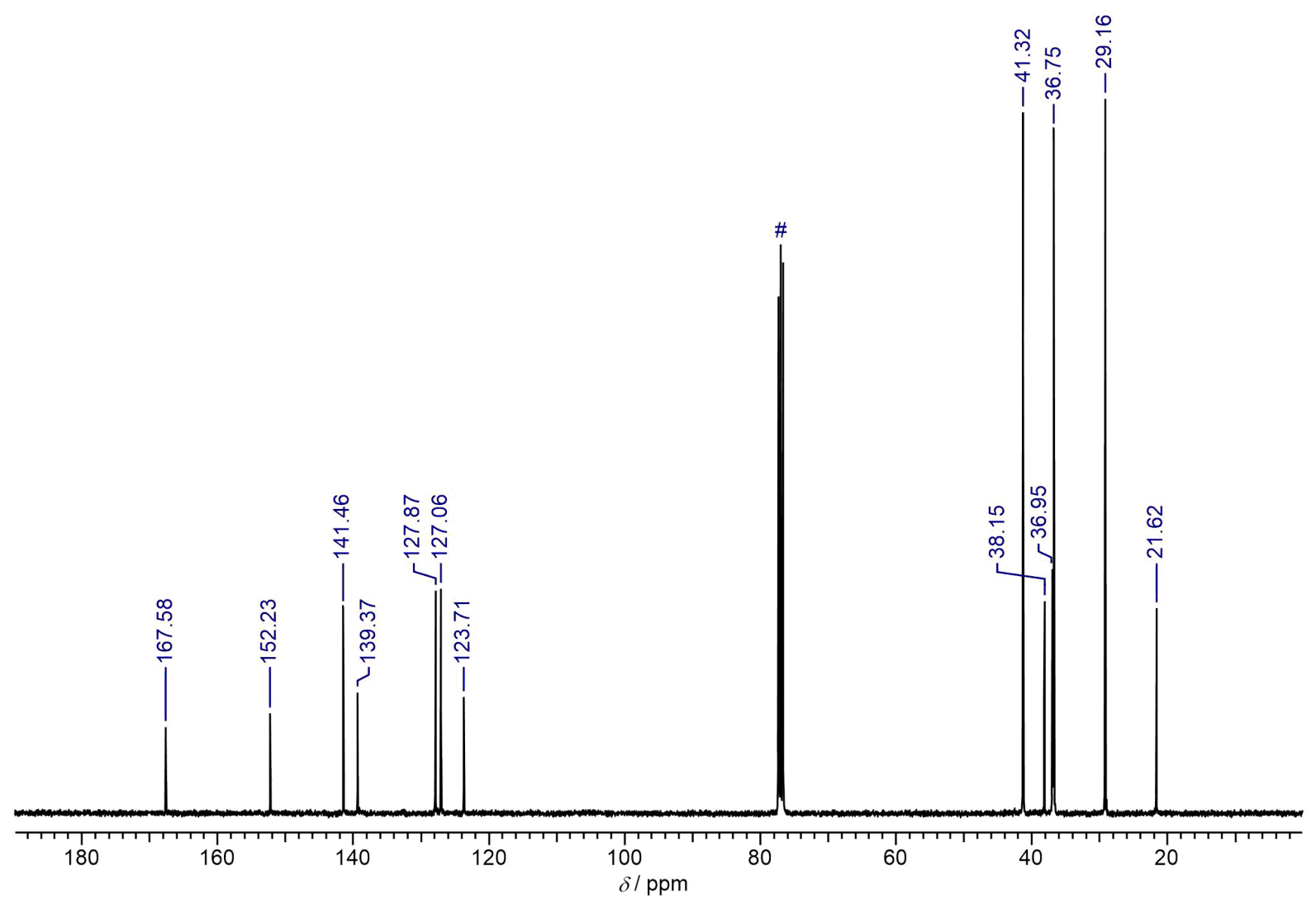

Figure S5: ${ }^{13} \mathrm{C}$ NMR of 3 recorded in chloroform- $d_{3}(\#)$. 


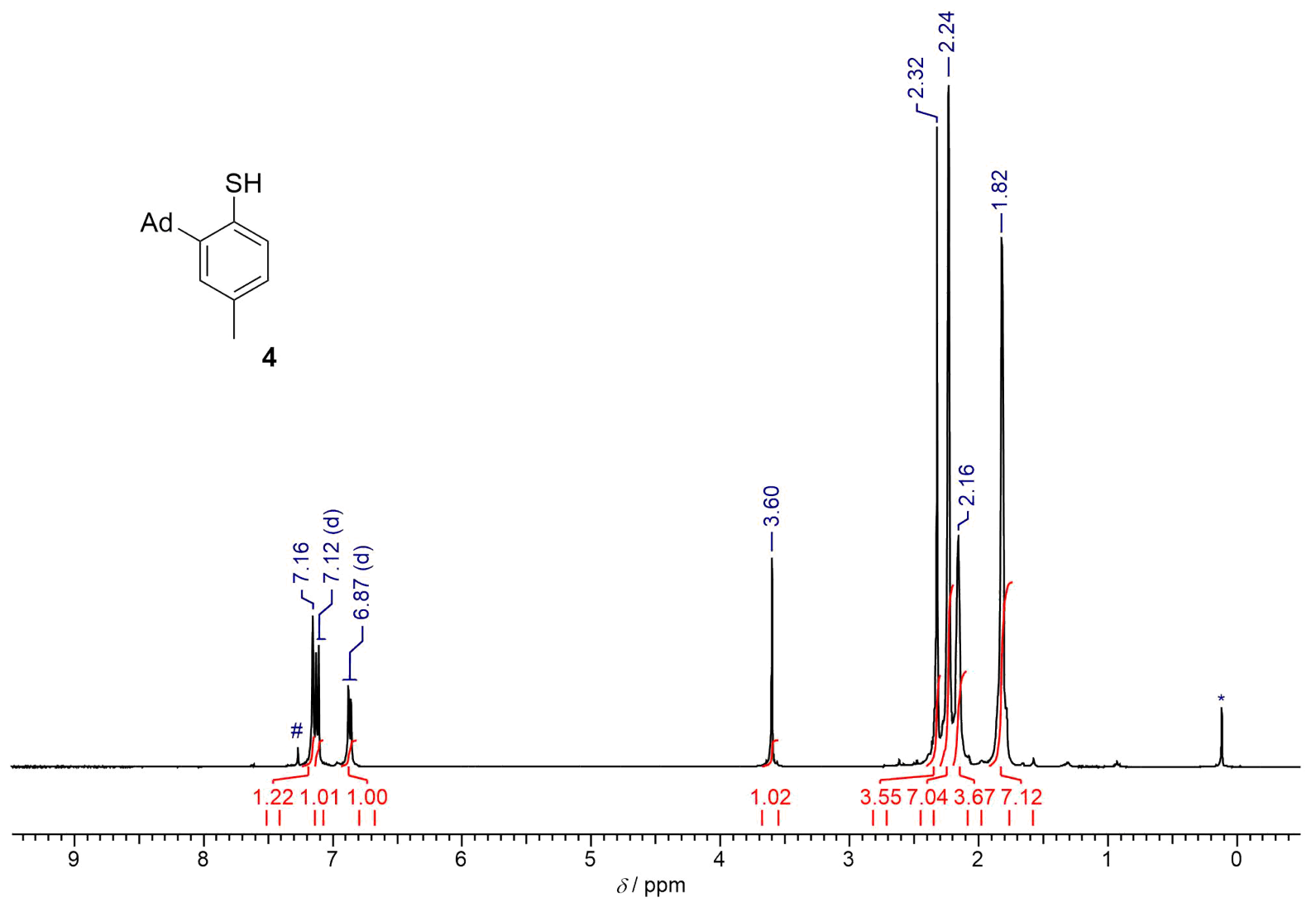

Figure S6: ${ }^{1} \mathrm{H}$ NMR of 4 recorded in chloroform- $d_{3}(\#)$. Residual silicone grease is marked with an asterisk $(*)$.

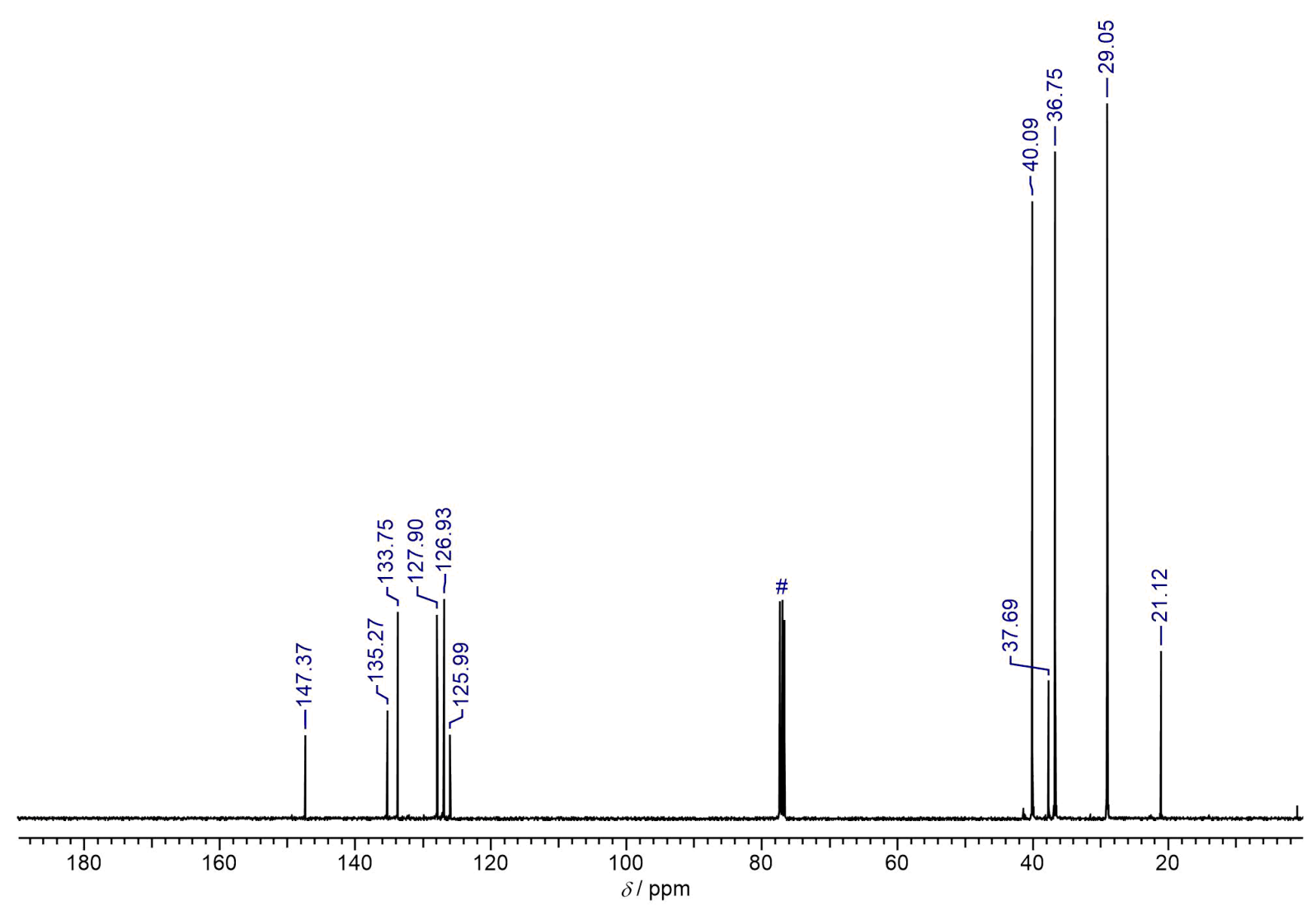

Figure S7: ${ }^{13} \mathrm{C}$ NMR of 4 recorded in chloroform- $d_{3}(\#)$. 


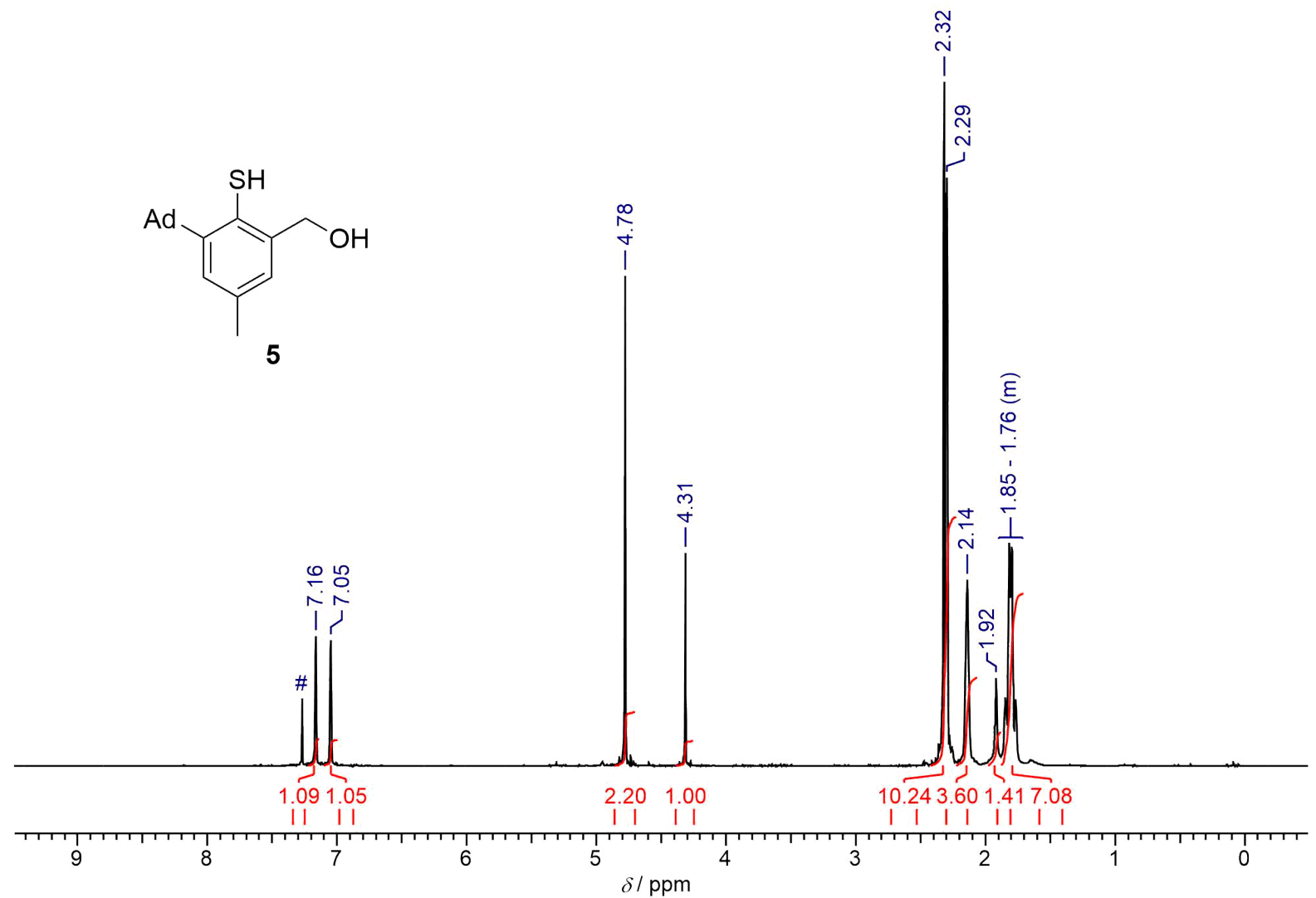

Figure S8: ${ }^{1} \mathrm{H}$ NMR of 5 recorded in chloroform- $d_{3}(\#)$.

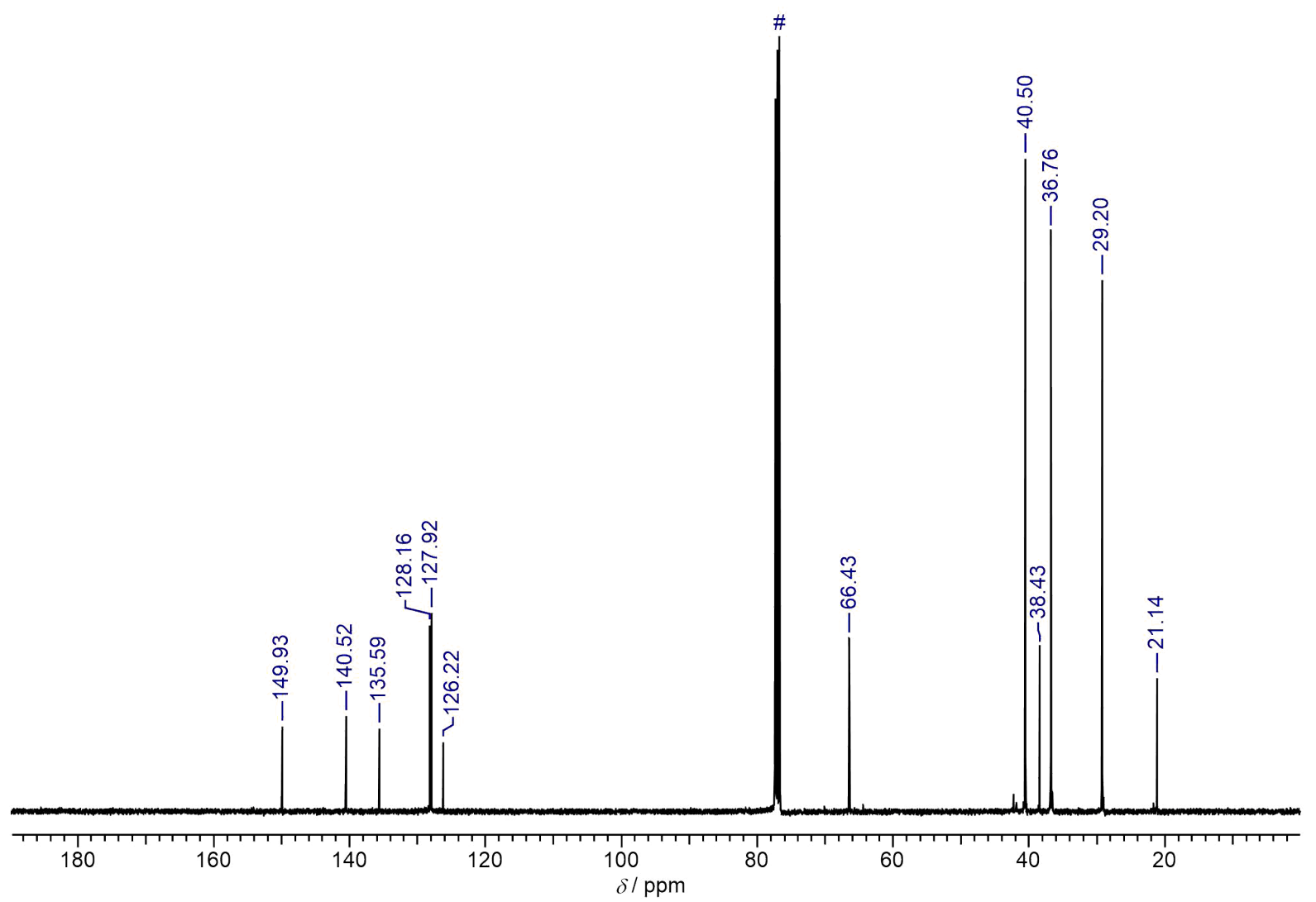

Figure S9: ${ }^{13} \mathrm{C}$ NMR of 5 recorded in chloroform- $d_{3}(\#)$. 

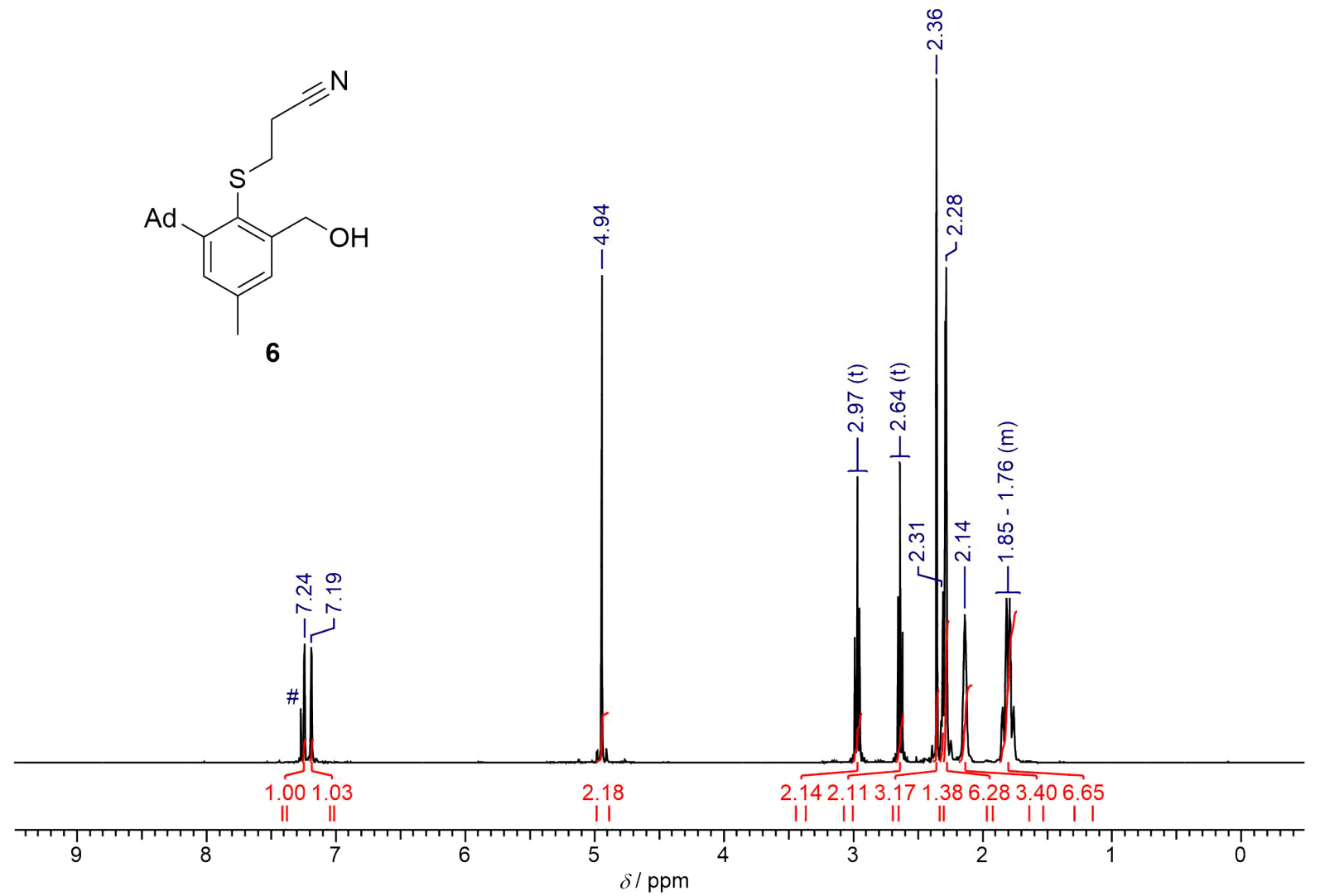

Figure S10: ${ }^{1} \mathrm{H}$ NMR of 6 recorded in chloroform- $d_{3}(\#)$.

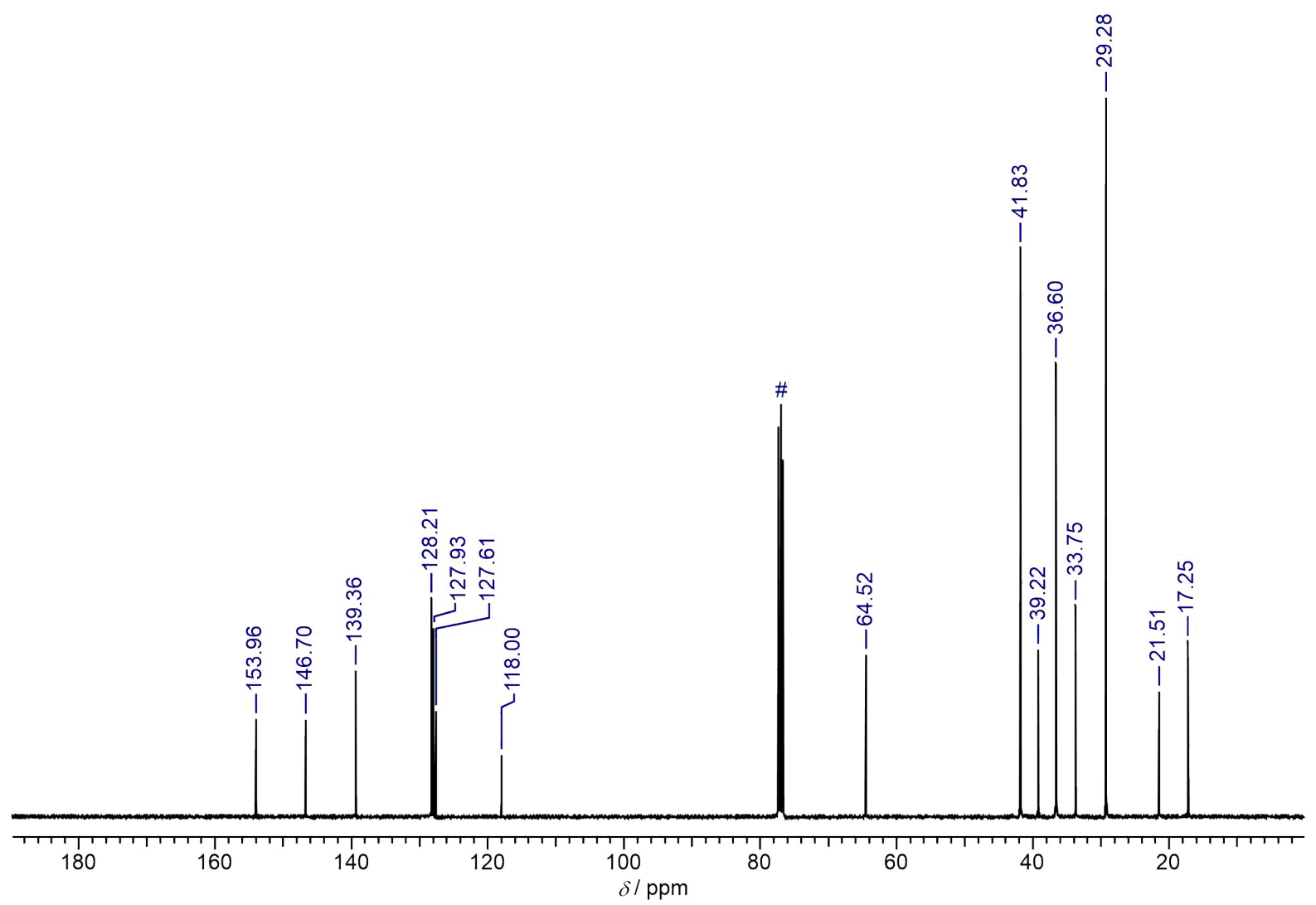

Figure S11: ${ }^{13} \mathrm{C}$ NMR of 6 recorded in chloroform- $d_{3}(\#)$. 

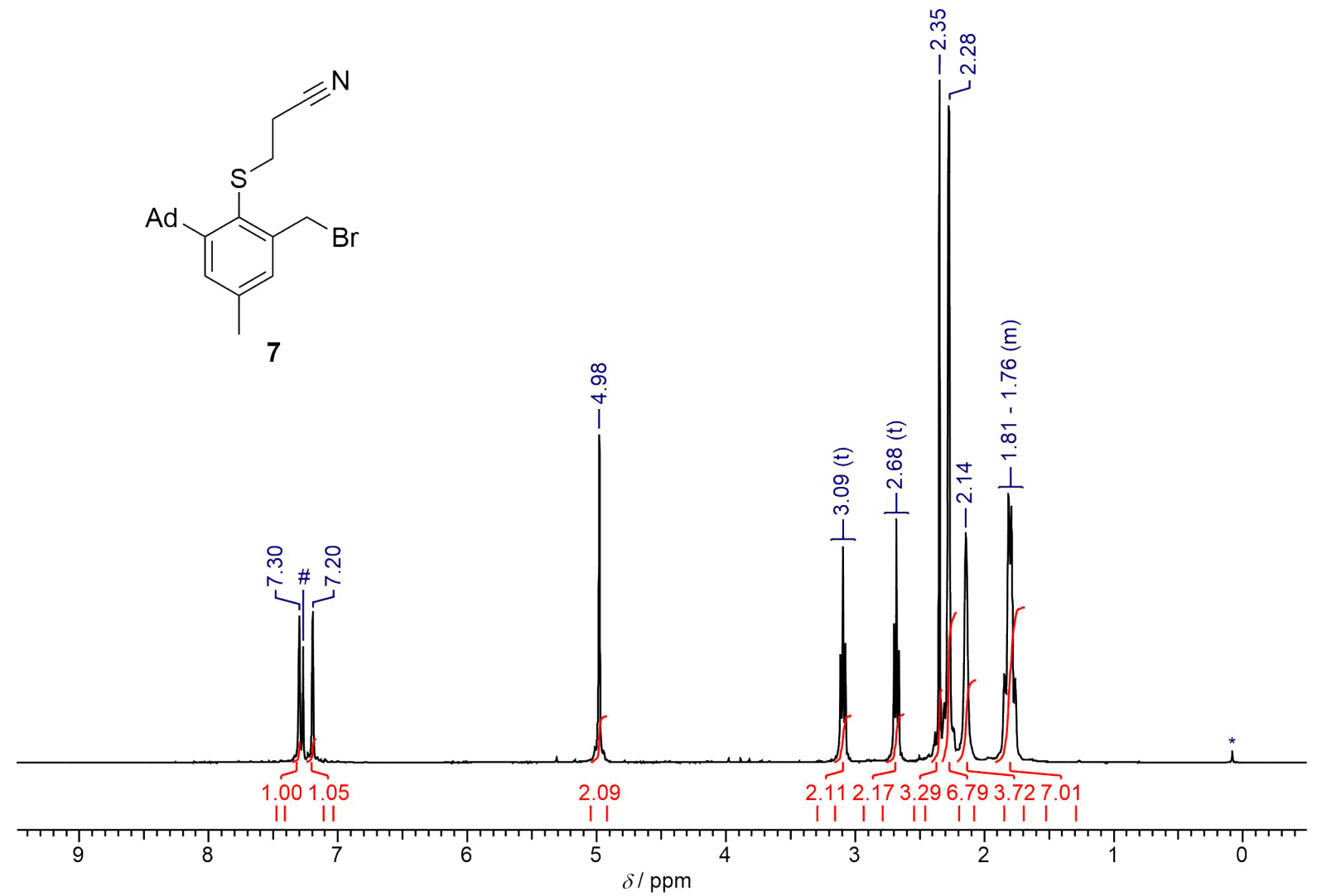

Figure S12: ${ }^{1} \mathrm{H}$ NMR of 7 recorded in chloroform- $d_{3}(\#)$. Residual silicone grease is marked with an asterisk $(*)$.

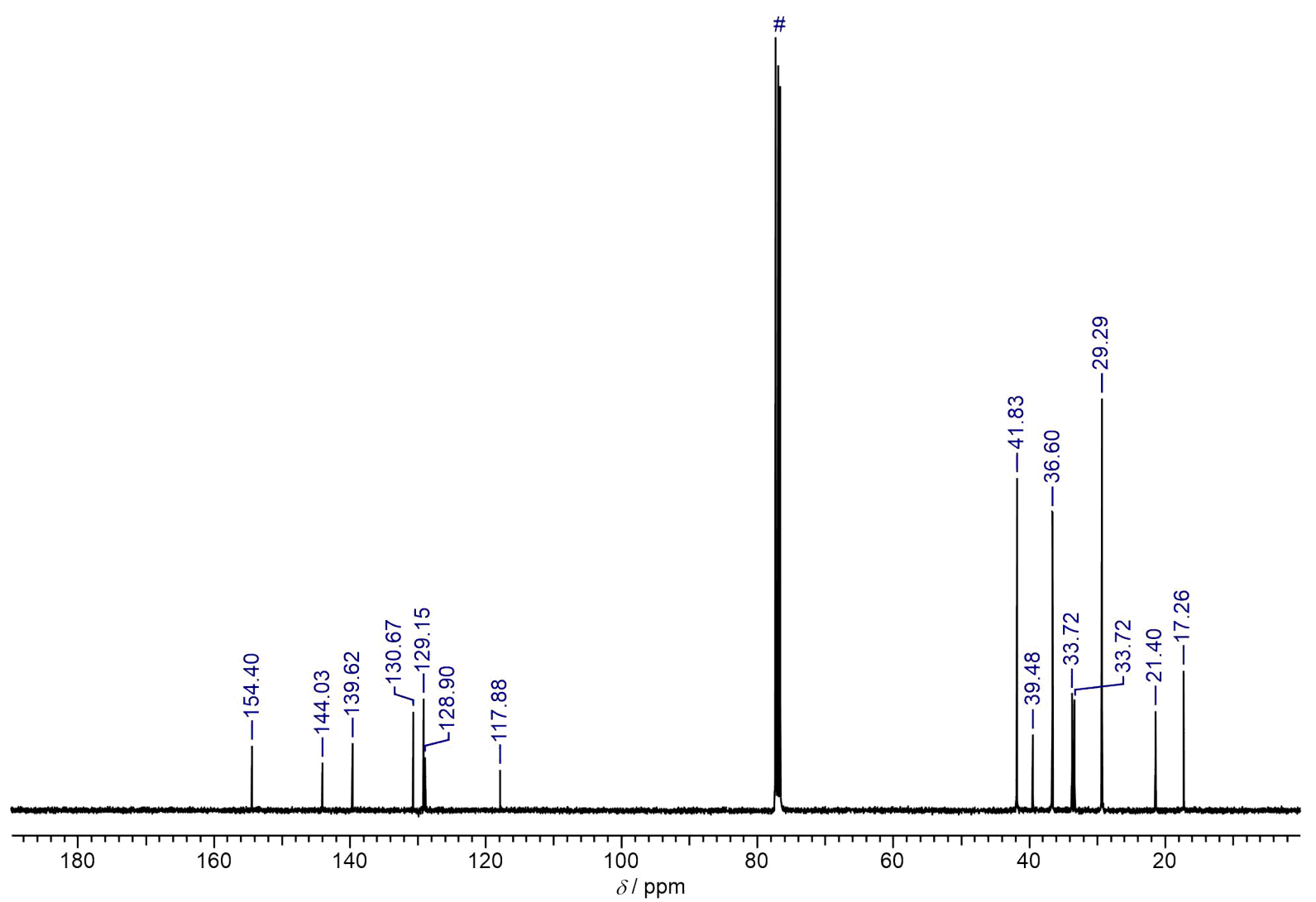

Figure S13: ${ }^{13} \mathrm{C}$ NMR of 7 recorded in chloroform- $d_{3}(\#)$. 
<smiles>Cc1cc(Cl)c(SCCC#N)c(Cc2c(C)c(Cc3cc(C)cc(Cl)c3SCCC#N)c(C)c(Cc3cc(C)cc([Ge])c3SCCC#N)c2C)c1</smiles>

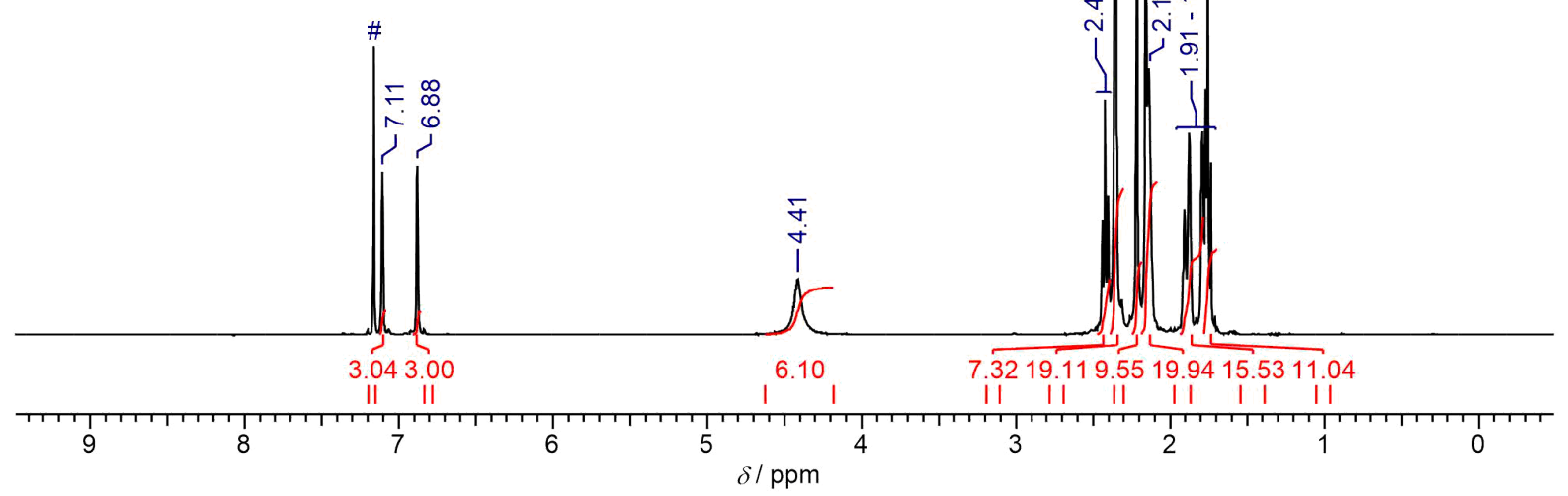

Figure S14: ${ }^{1} \mathrm{H}$ NMR of 8 recorded in chloroform- $d_{3}(\#)$.

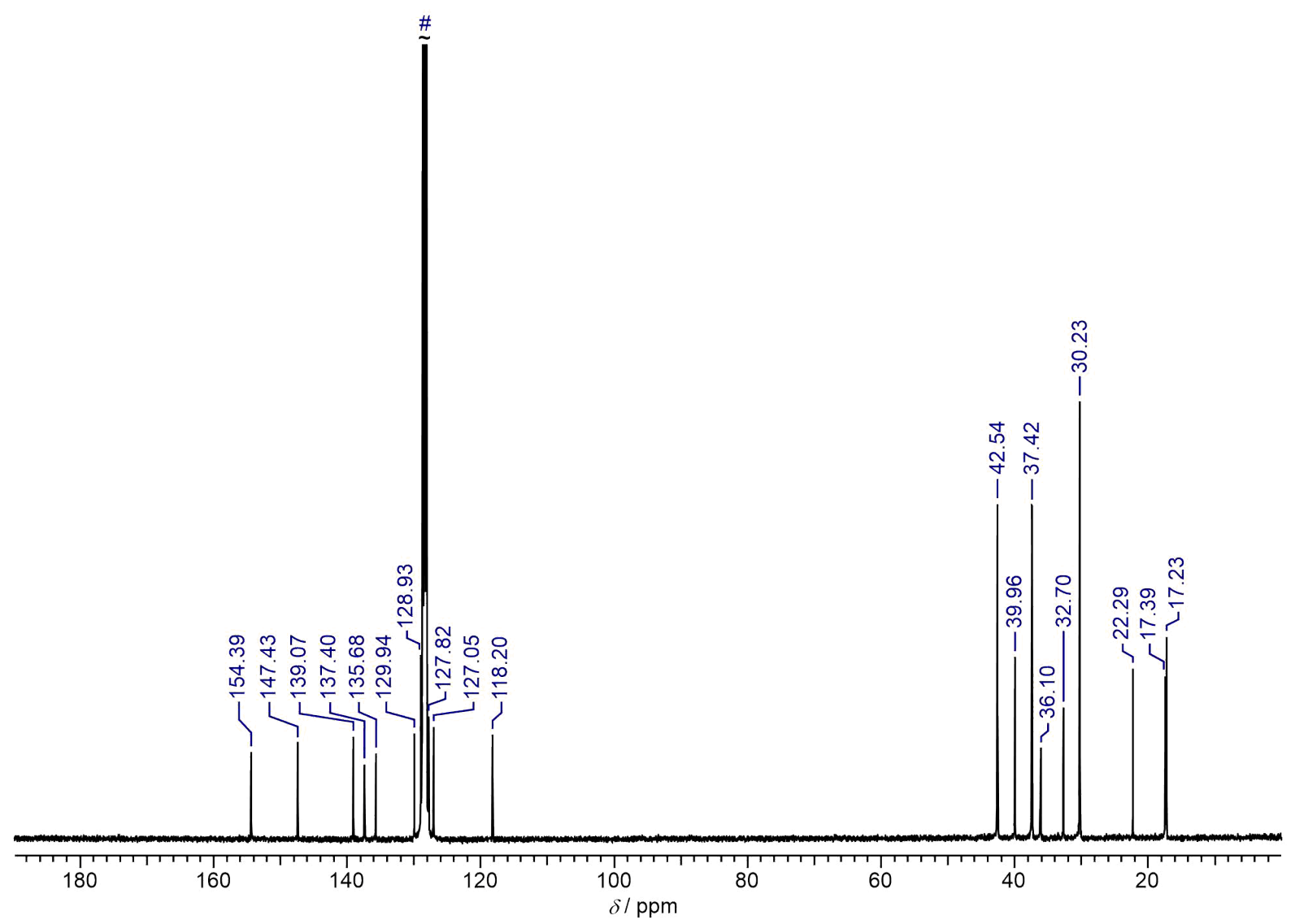

Figure S15: ${ }^{13} \mathrm{C}$ NMR of $\mathbf{8}$ recorded in chloroform- $d_{3}(\#)$. 


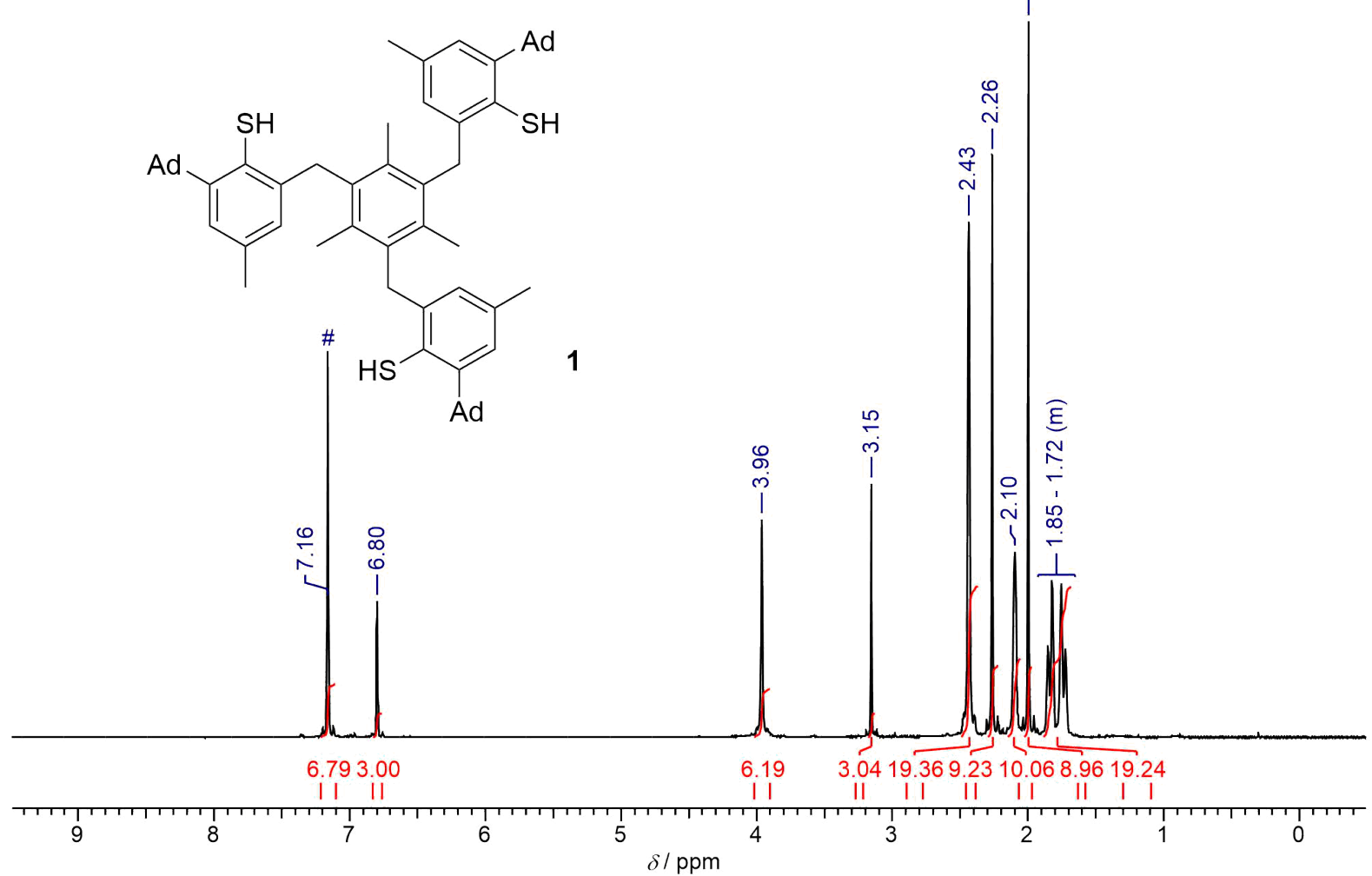

Figure S16: ${ }^{1} \mathrm{H}$ NMR of 1 recorded in benzene- $d_{6}(\#)$.

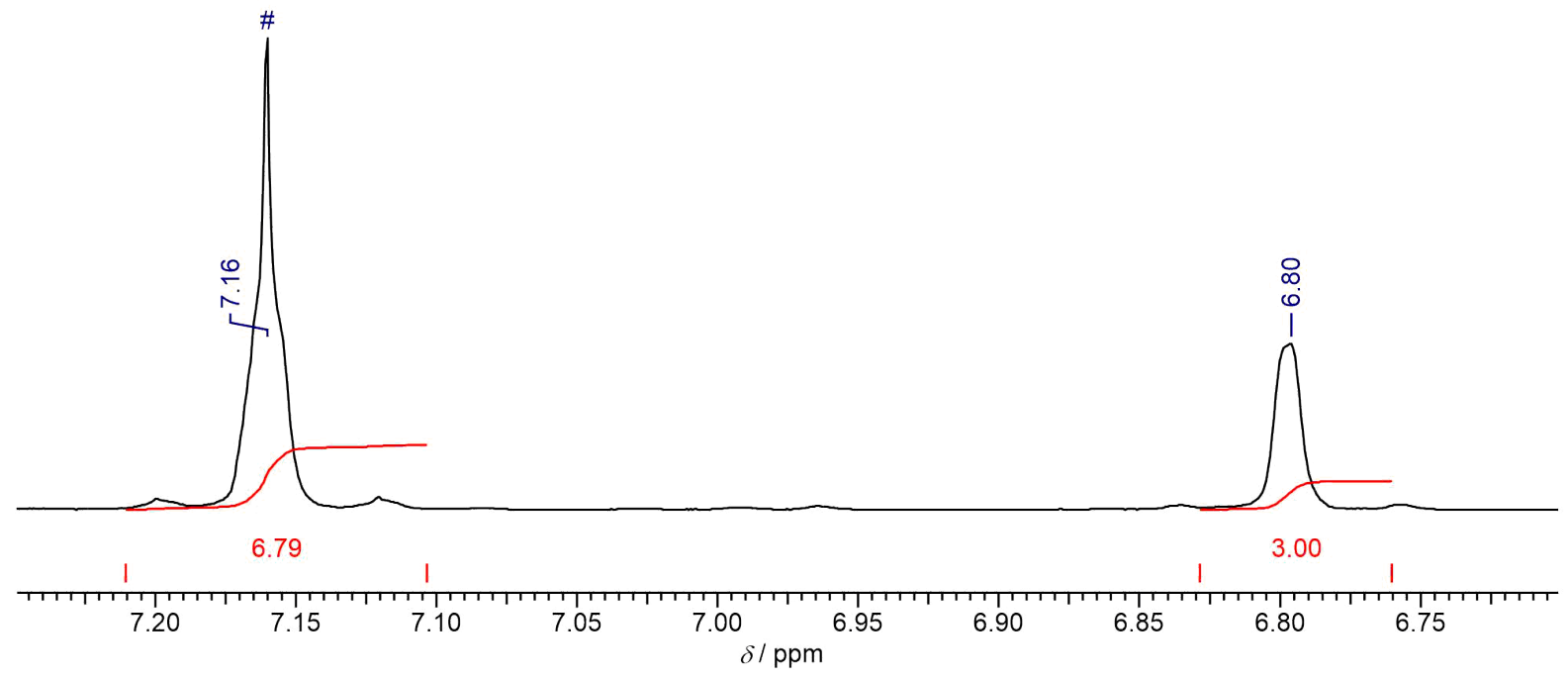

Figure S17: ${ }^{1} \mathrm{H}$ NMR of 1 recorded in benzene- $d_{6}(\#)$. Zoom into the aromatic region. 


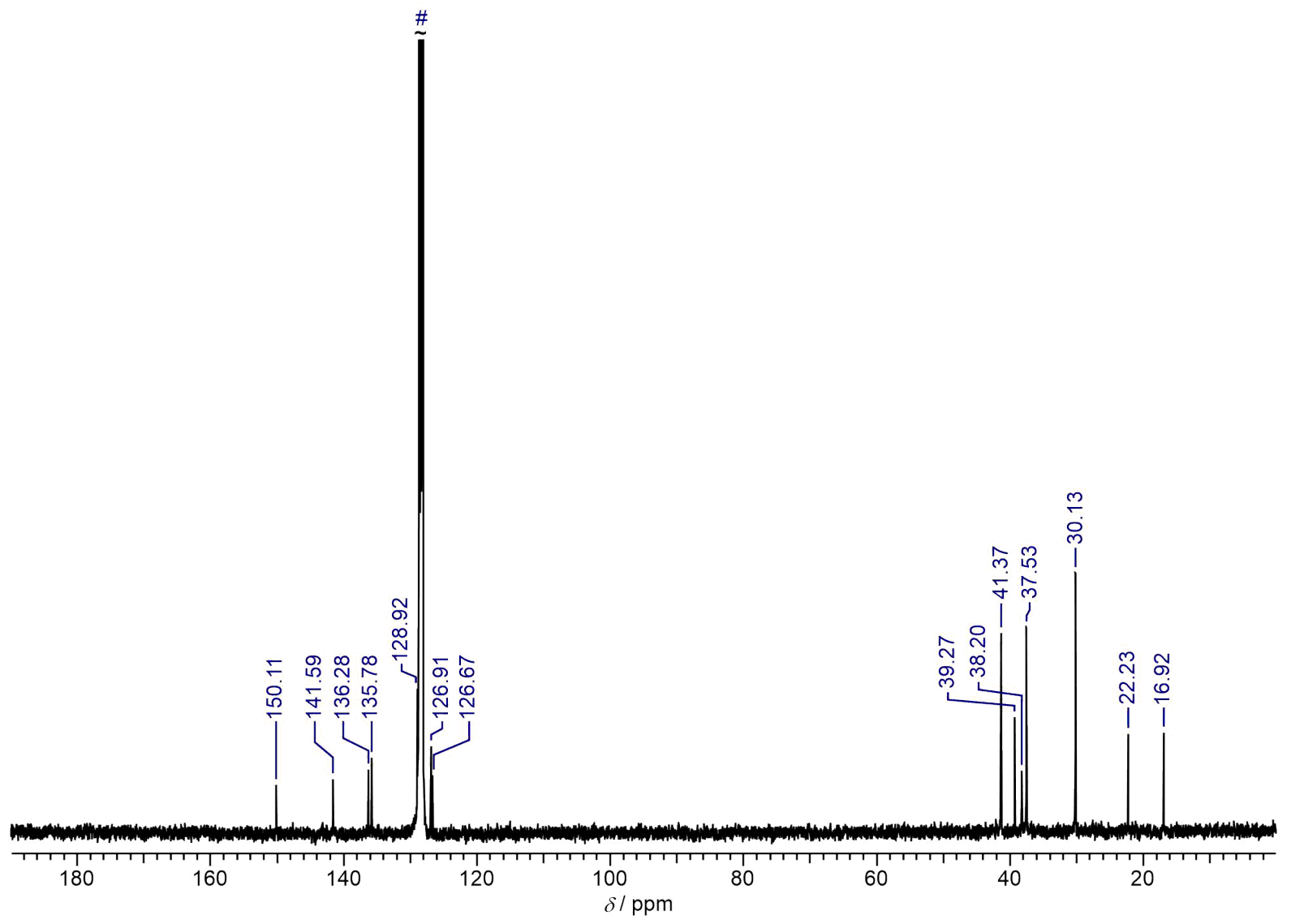

Figure S18: ${ }^{13} \mathrm{C}$ NMR of 1 recorded in benzene- $d_{6}(\#)$.

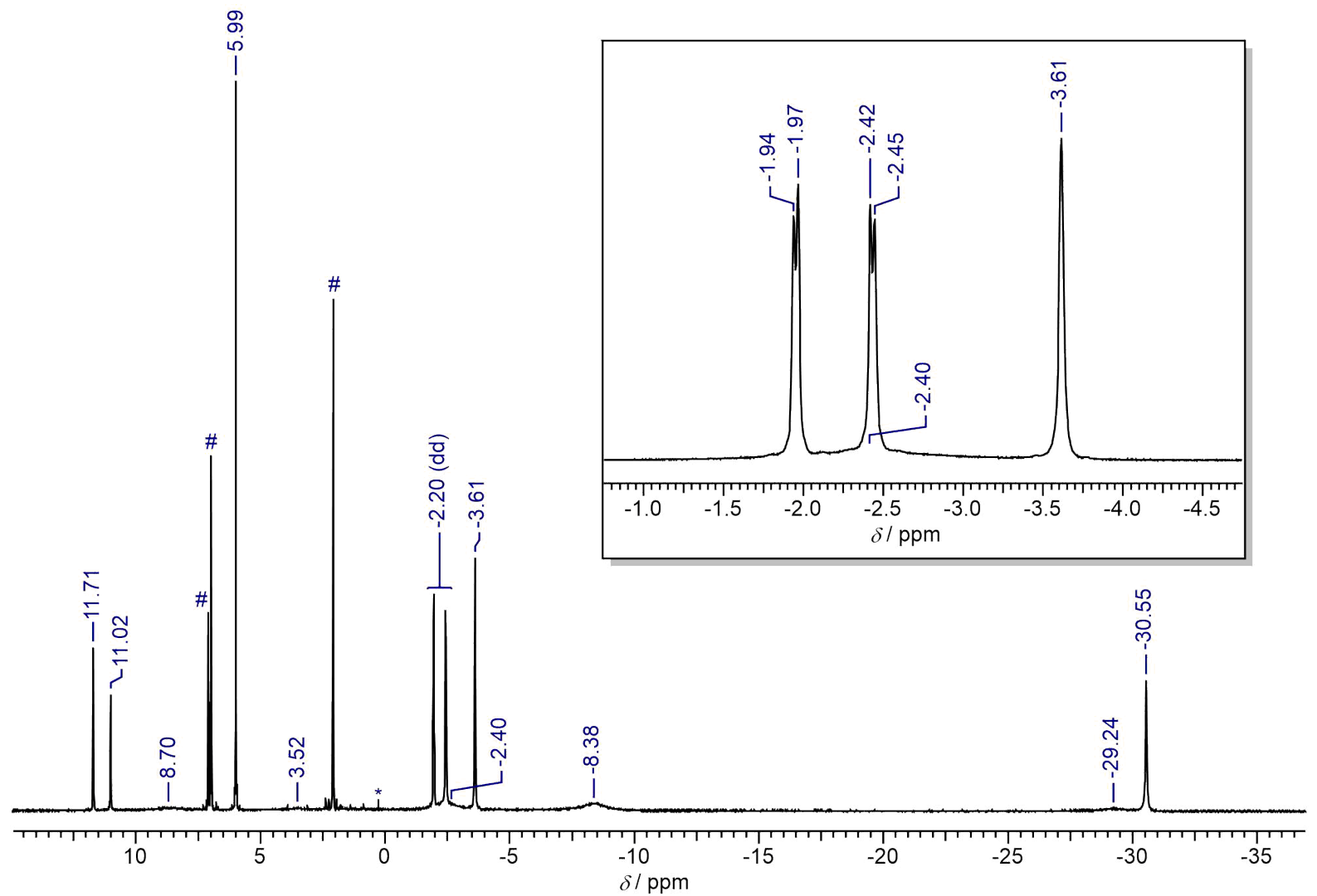

Figure S19: ${ }^{1} \mathrm{H}$ NMR of 1-U recorded in toluene- $d_{8}(\#)$. Residual silicone grease is marked with an asterisk $(*)$. 


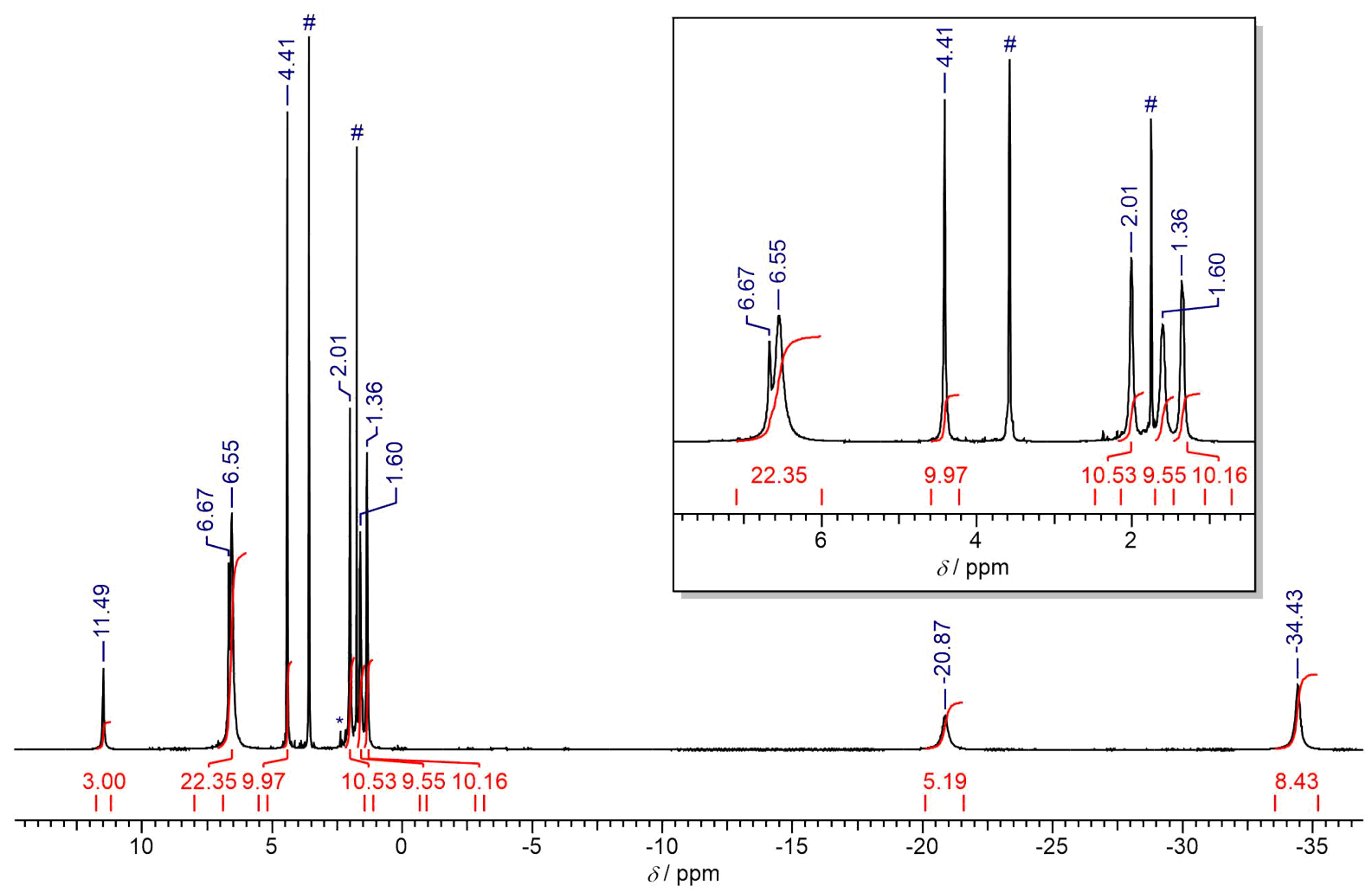

Figure S20: ${ }^{1} \mathrm{H}$ NMR of 1-U recorded in tetrahydrofuran- $d_{8}(\#)$. Residual free ligand $\mathbf{1}$ is marked with an asterisk $(*)$. 
IR Vibrational Spectroscopy

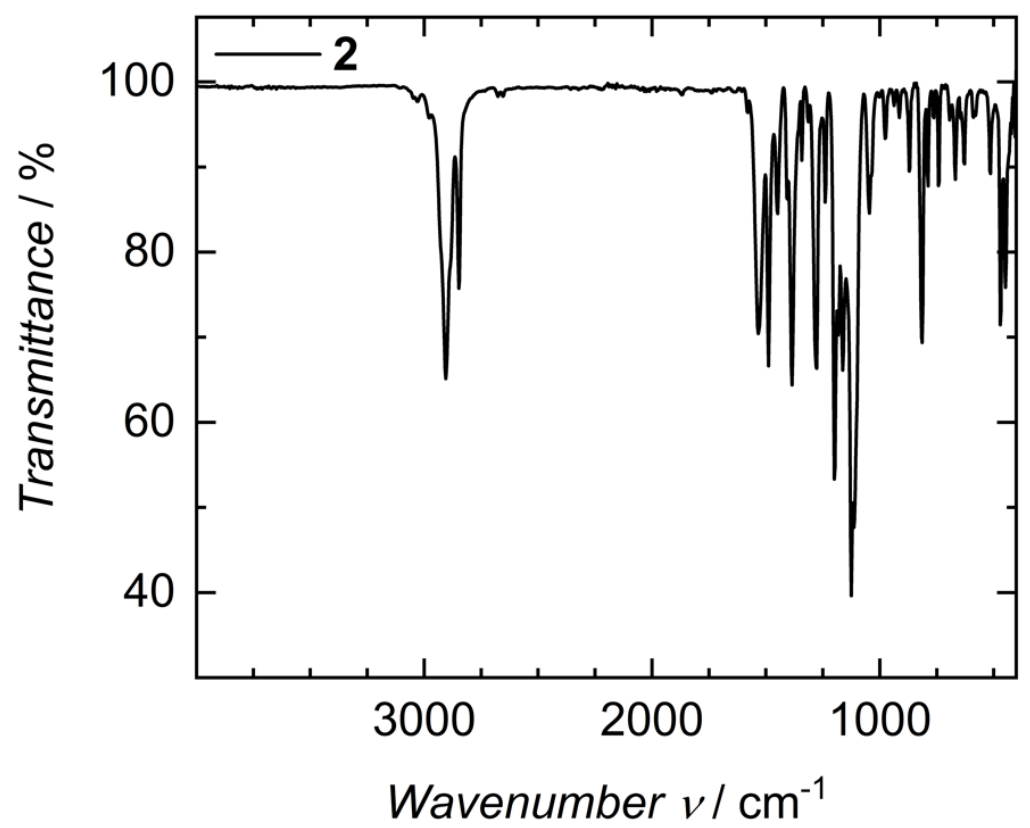

Figure S21: IR vibrational spectrum of 2, recorded as neat powder on an ATR-IR instrument.

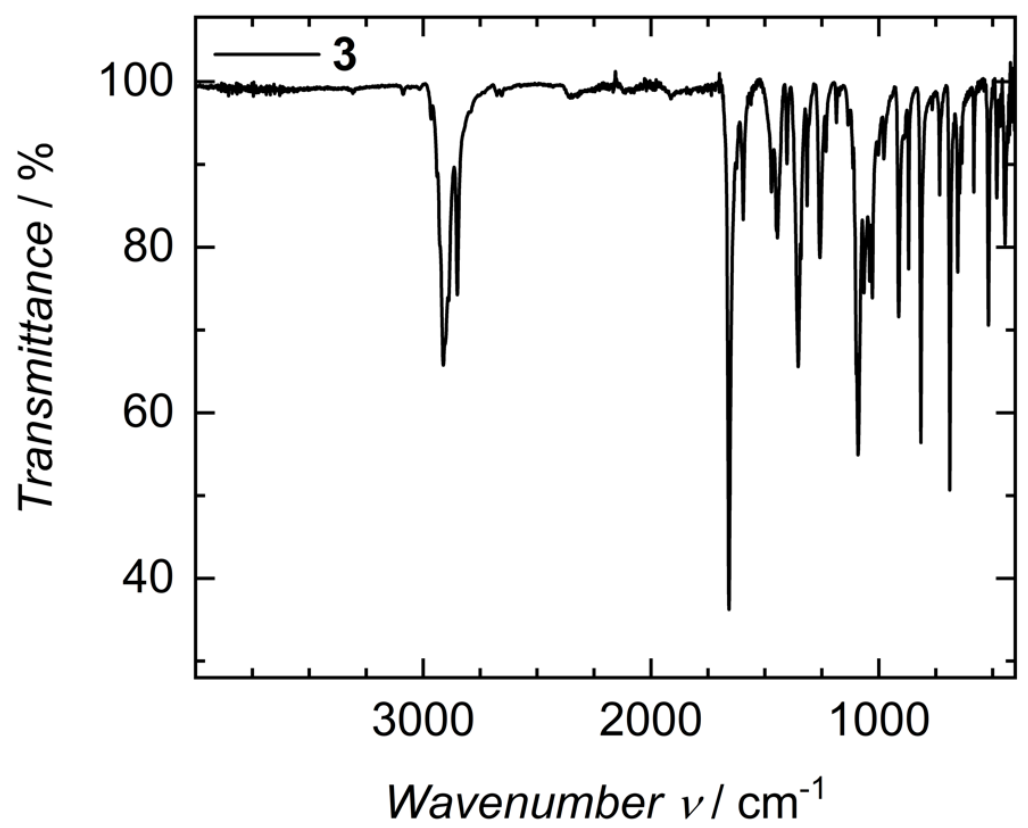

Figure S22: IR vibrational spectrum of $\mathbf{3}$, recorded as neat powder on an ATR-IR instrument. 


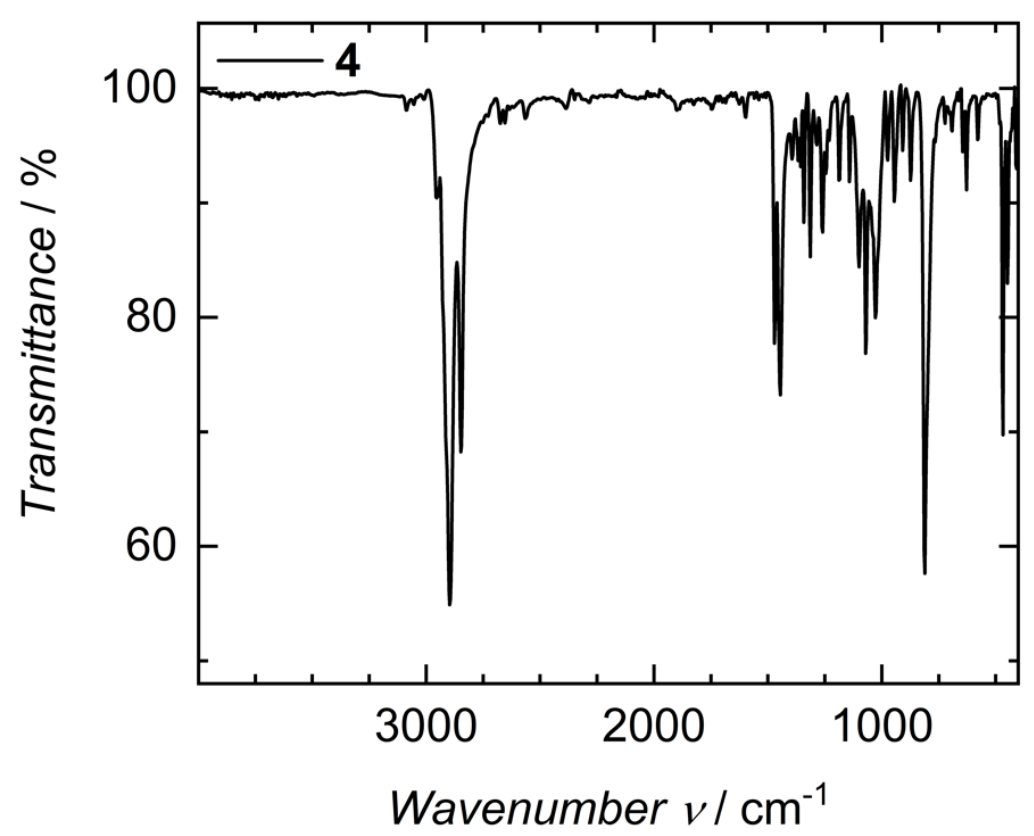

Figure S23: IR vibrational spectrum of 4, recorded as neat powder on an ATR-IR instrument.

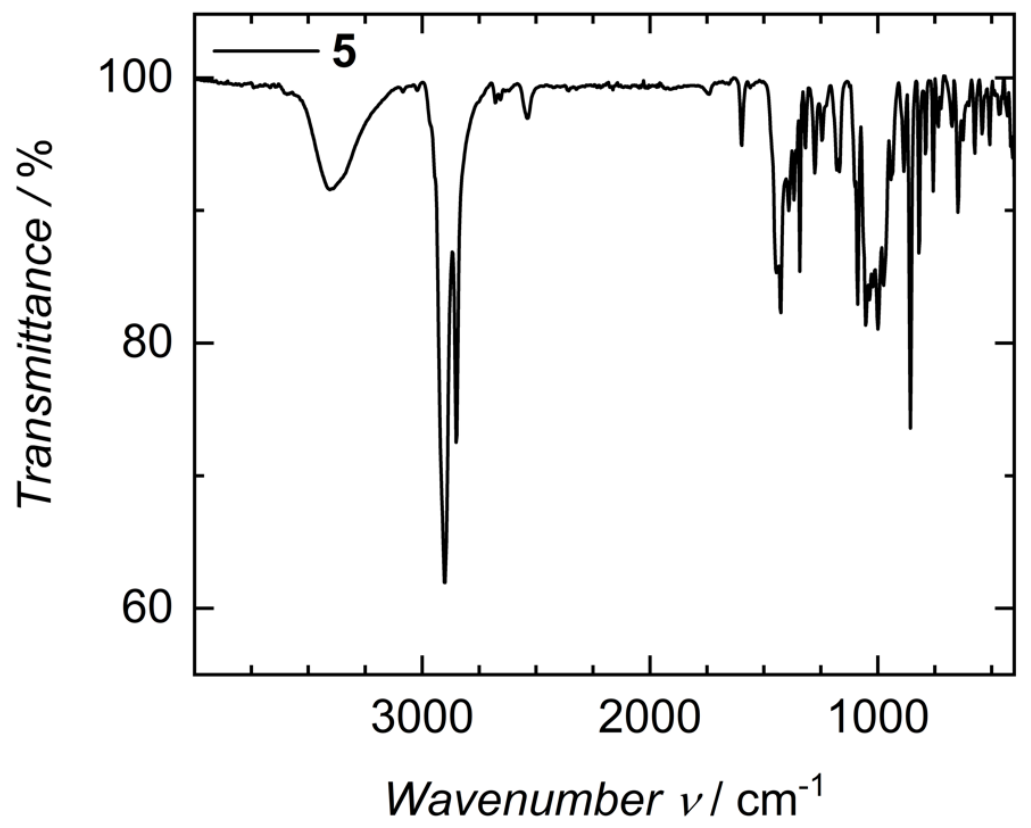

Figure S24: IR vibrational spectrum of $\mathbf{5}$, recorded as neat powder on an ATR-IR instrument. 


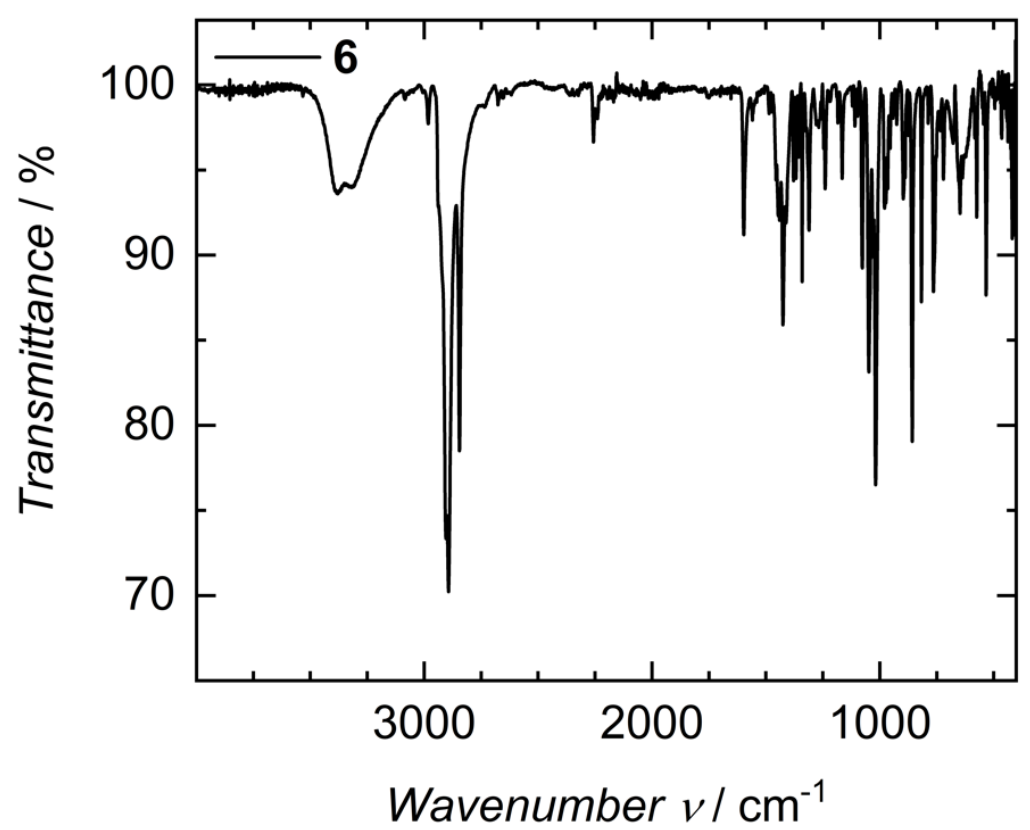

Figure S25: IR vibrational spectrum of 6, recorded as neat powder on an ATR-IR instrument.

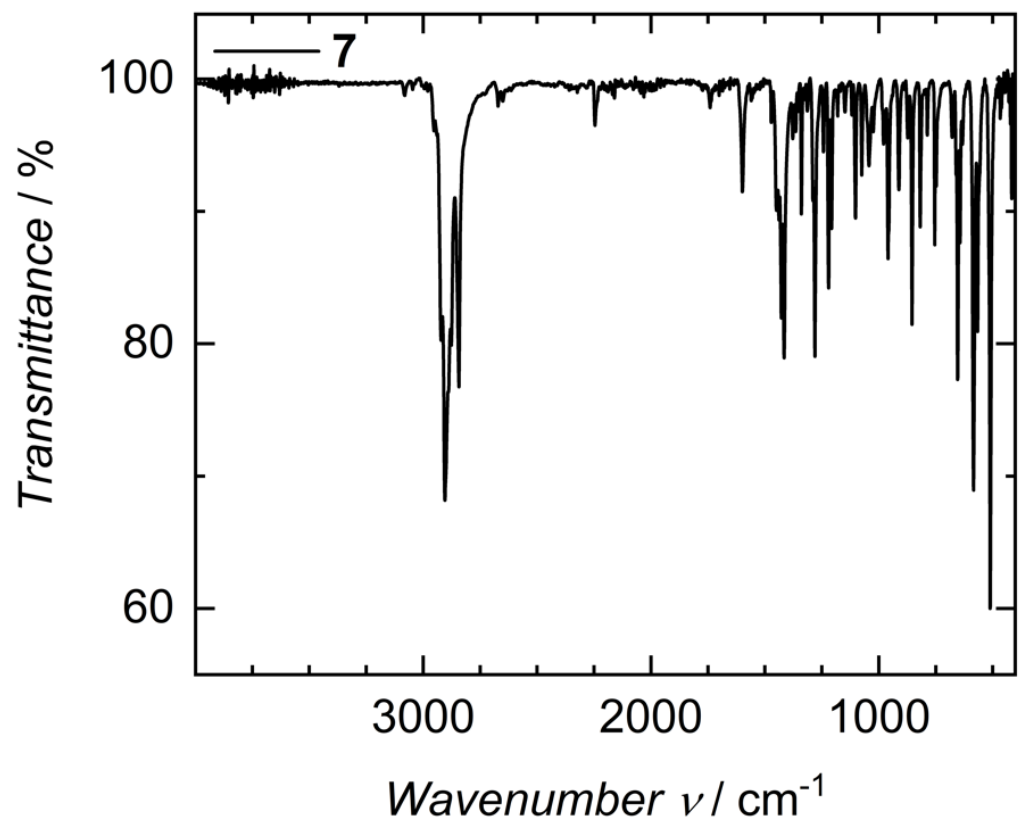

Figure S26: IR vibrational spectrum of 7, recorded as neat powder on an ATR-IR instrument. 


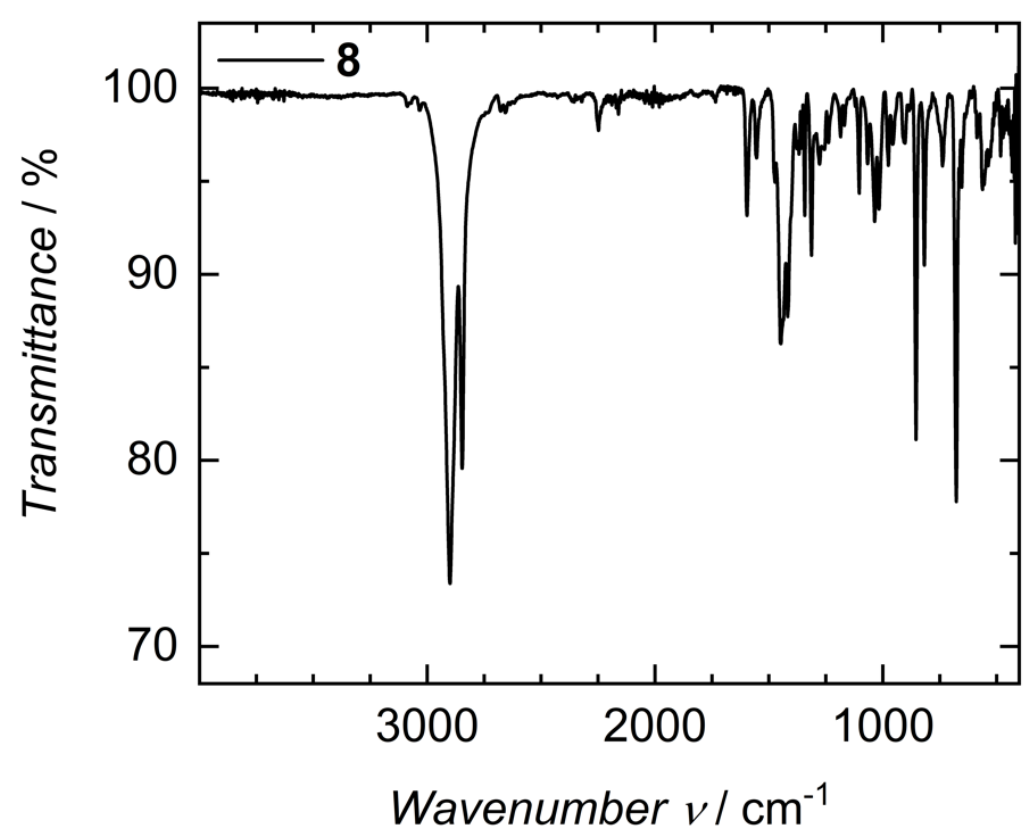

Figure S27: IR vibrational spectrum of $\mathbf{8}$, recorded as neat powder on an ATR-IR instrument.

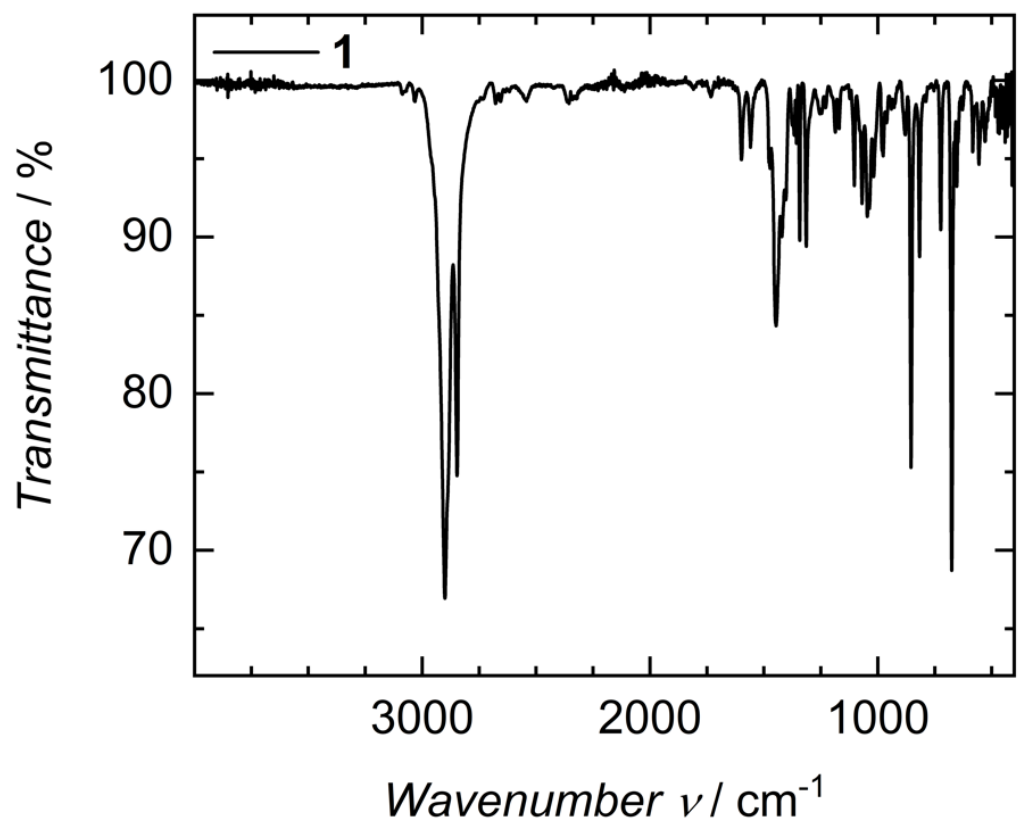

Figure S28: IR vibrational spectrum of 1, recorded as neat powder on an ATR-IR instrument. 


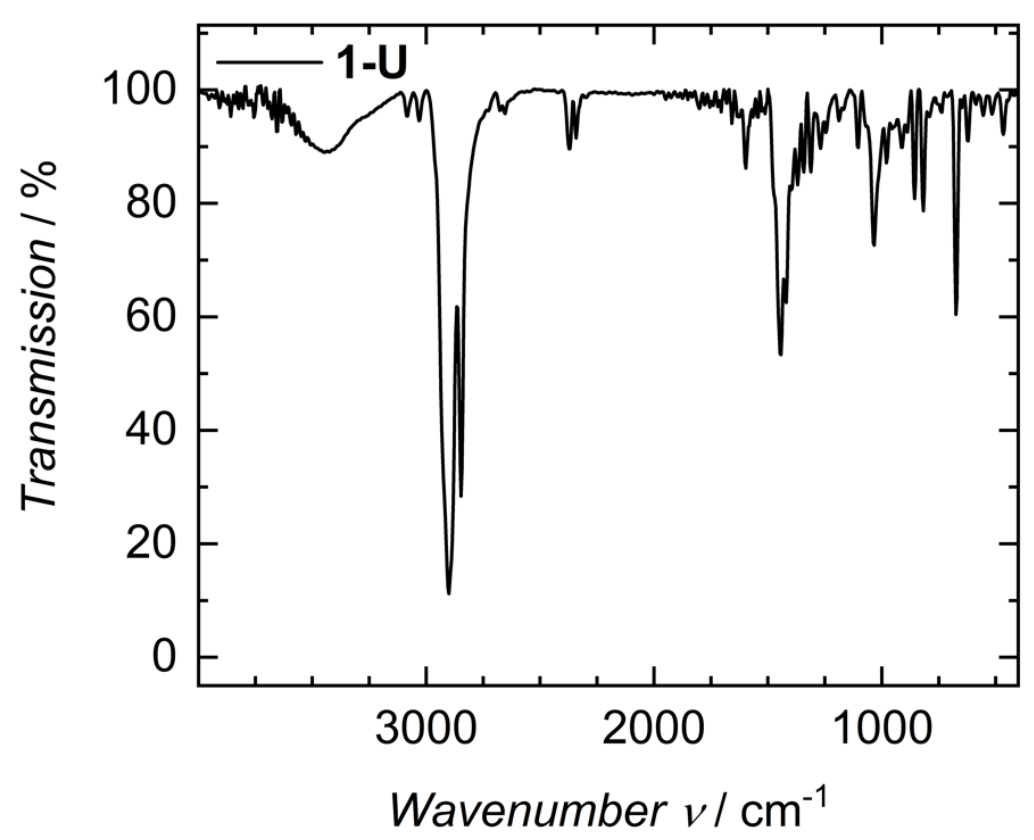

Figure S29: IR vibrational spectrum of $\mathbf{1 - U}$ recorded in a $\mathrm{KBr}$ pellet at room temperature.

\section{UV/Vis/NIR Spectroscopy}

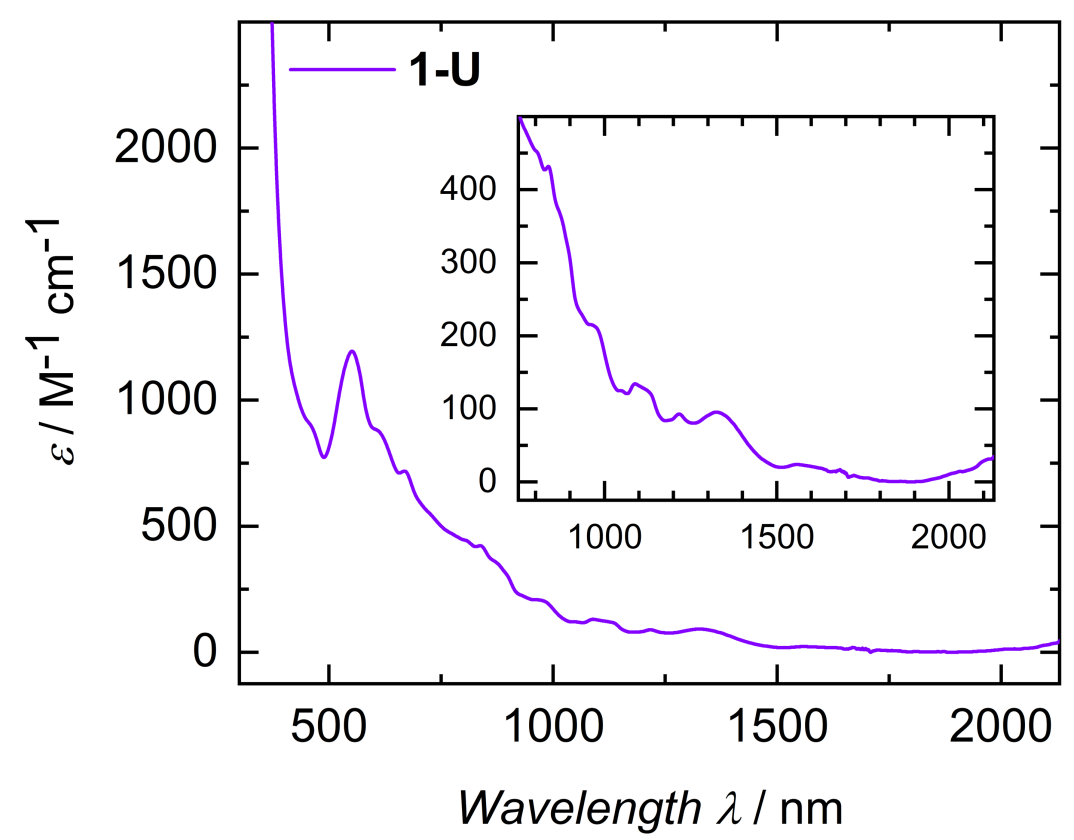

Figure S30: Vis-NIR electronic absorption spectrum of complex 1-U recorded at a concentration of $7.1 \cdot 10^{-4} \mathrm{M}$ in toluene, with inset zoom into the NIR area recorded at a concentration of $2.0 \cdot 10^{-3} \mathrm{M}$ in toluene. 


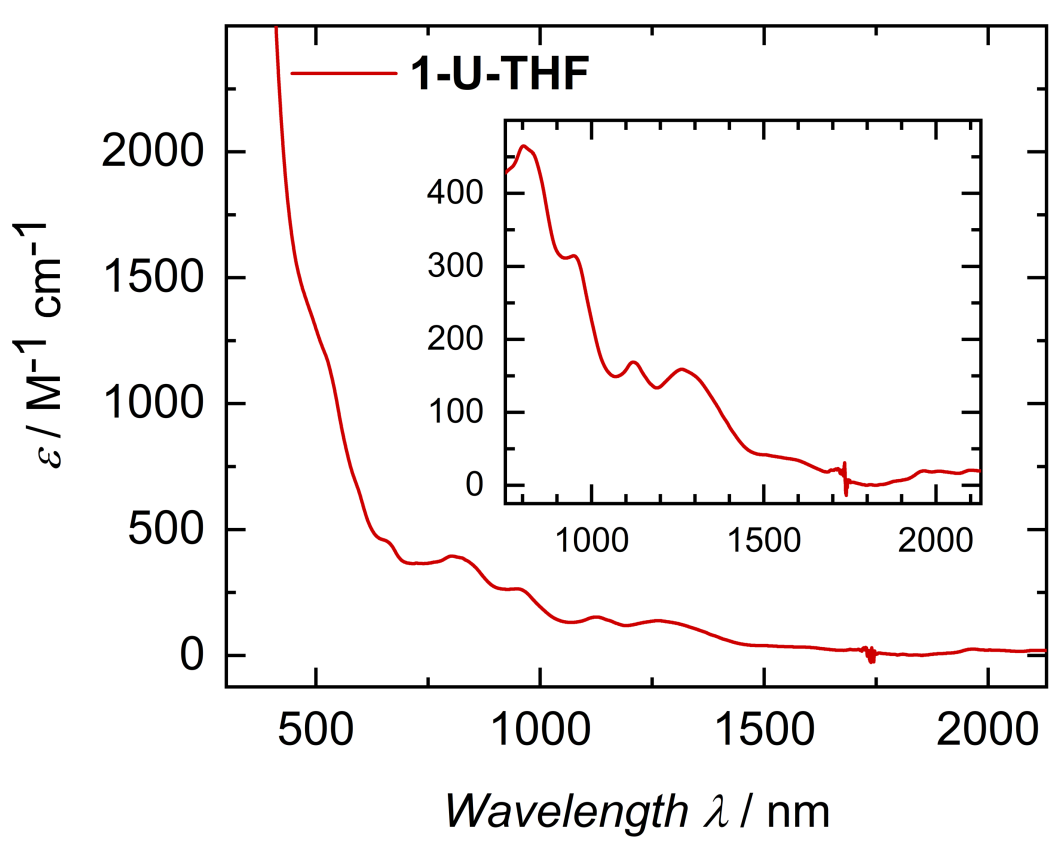

Figure S31: Vis-NIR electronic absorption spectrum of complex 1-U-THF recorded at a concentration of $8.7 \cdot 10^{-4} \mathrm{M}$ in tetrahydrofuran, with inset zoom into the NIR area recorded at a concentration of $2.4 \cdot 10^{-3} \mathrm{M}$ in tetrahydrofuran.

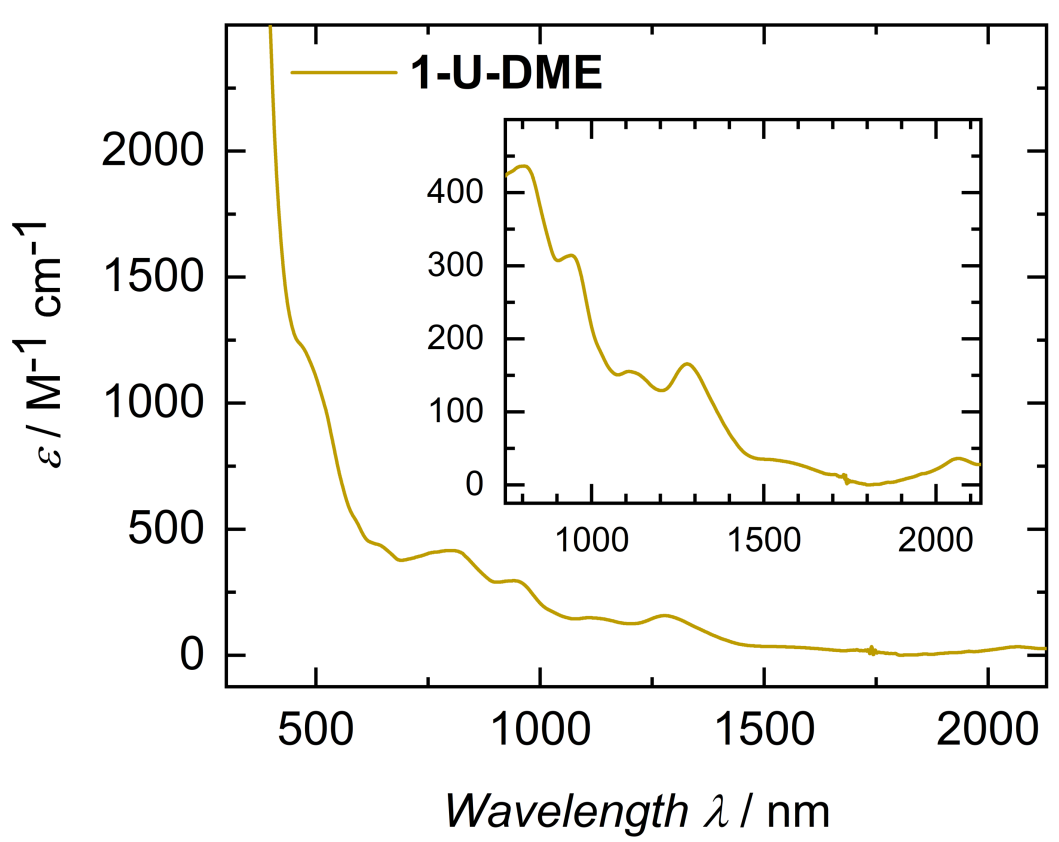

Figure S32: Vis-NIR electronic absorption spectrum of complex 1-U-DME recorded at a concentration of $8.0 \cdot 10^{-4} \mathrm{M}$ in 1,2-dimethoxyethane, with inset zoom into the NIR area recorded at a concentration of $2.4 \cdot 10^{-3} \mathrm{M}$ in 1,2-dimethoxyethane. 


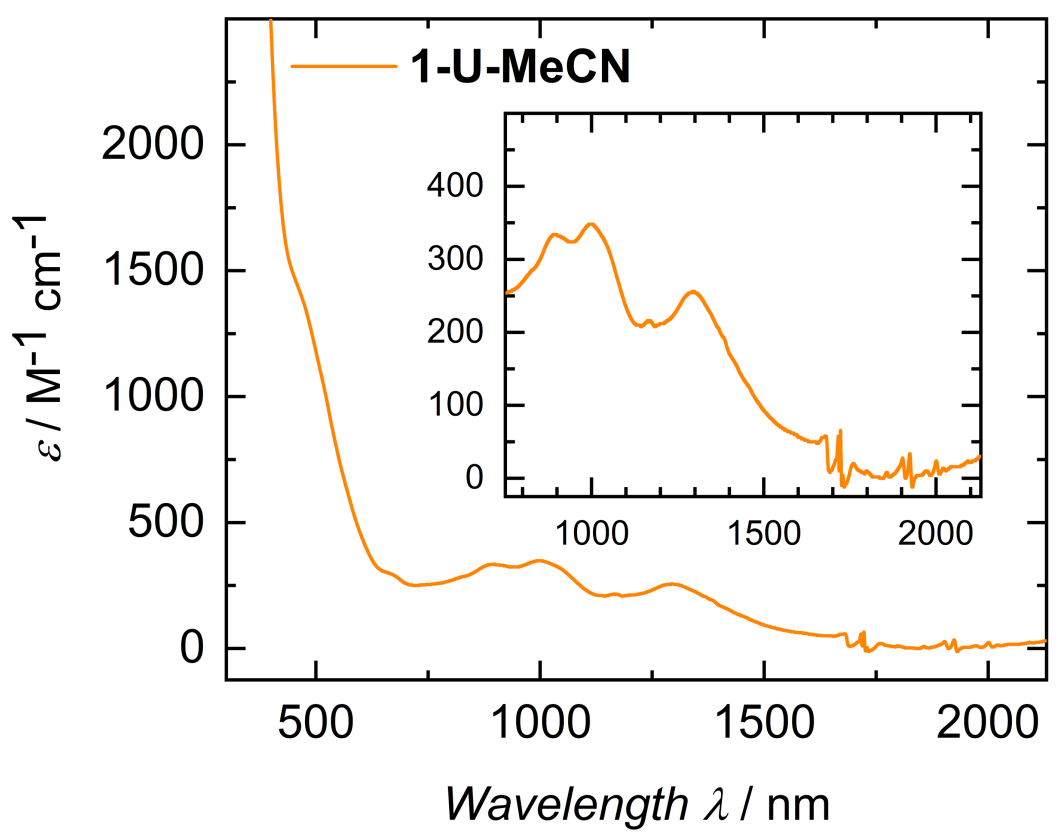

Figure S33: Vis-NIR electronic absorption spectrum of complex 1-U-MeCN and inset zoom into the NIR area, recorded at a concentration of $5.4 \cdot 10^{-4} \mathrm{M}$ in an acetonitrile/THF $(\mathrm{v} / \mathrm{v}=4: 1)$ mixture.

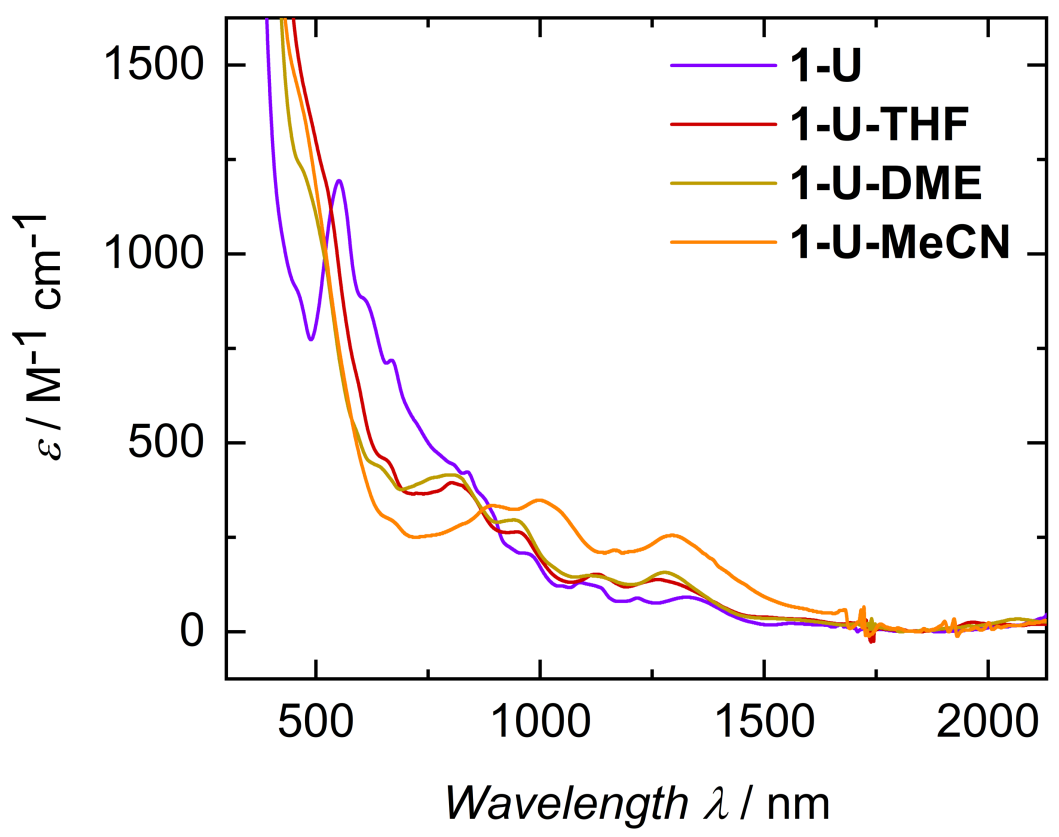

Figure S34: Comparison of UV/Vis-NIR electronic absorption spectra of complex 1-U recorded in different solvents: toluene $\left(\mathbf{1 - U}, 7.1 \cdot 10^{-4} \mathrm{M}\right)$, tetrahydrofuran $\left(\mathbf{1}-\mathbf{U}-\mathbf{T H F}, 8.7 \cdot 10^{-4} \mathrm{M}\right), \mathbf{1 , 2}$ dimethoxyethane (1-U-DME, $\left.8.0 \cdot 10^{-4} \mathrm{M}\right)$ and an acetonitrile/THF $4: 1$ mixture (1-U-MeCN, $\left.5.4 \cdot 10^{-4} \mathrm{M}\right)$. 


\section{Electrochemical Measurements}

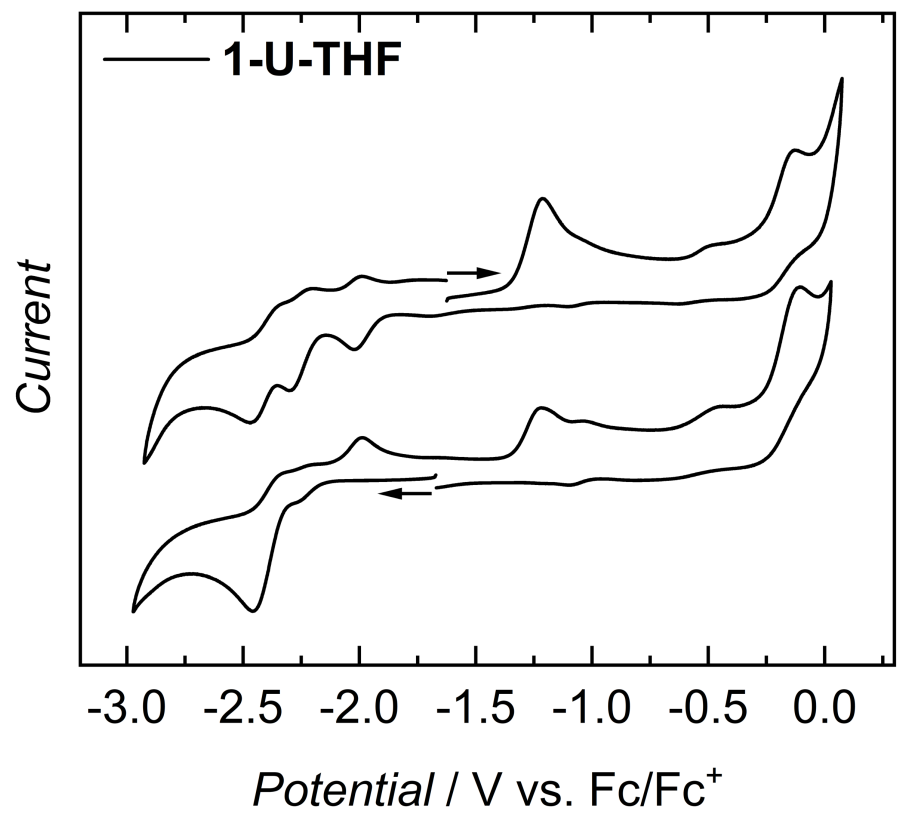

Figure S35: Cyclic voltammograms of $\mathbf{1 - U}$ measured in a THF solution with $0.1 \mathrm{M} \mathrm{TBAPF}_{6}$ as electrolyte, scan rate $200 \mathrm{mV} / \mathrm{s}$.

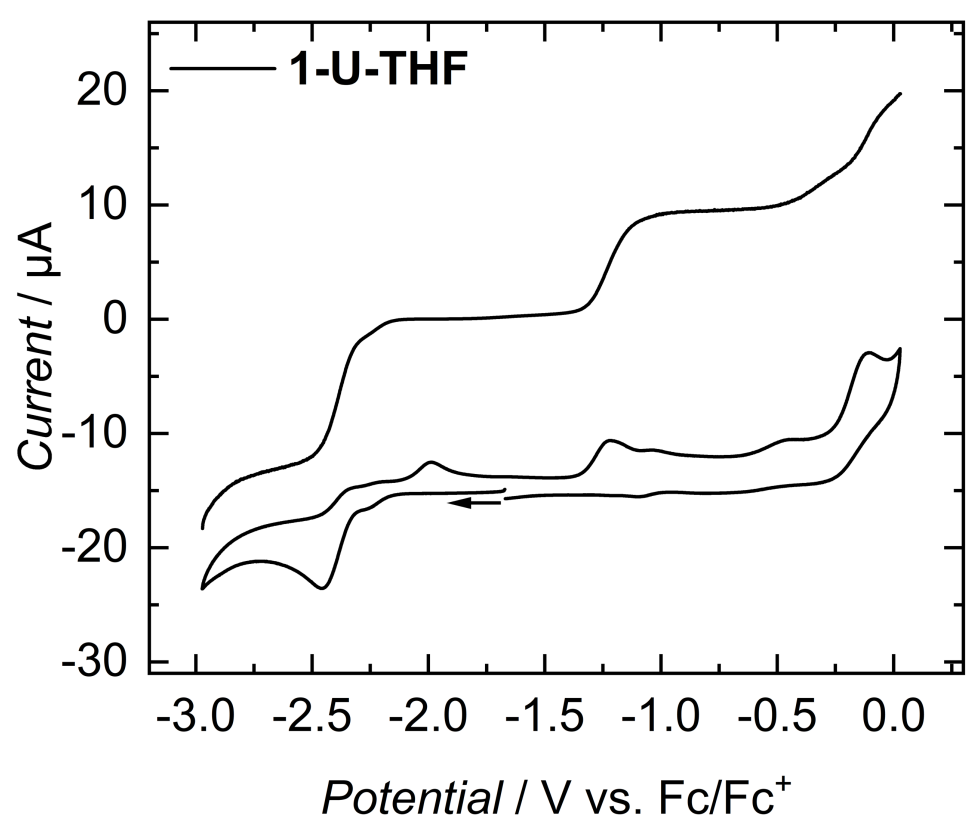

Figure S36: Linear sweep measurement (top) and cyclic voltammogram (bottom) of 1-U in a THF solution with $0.1 \mathrm{M} \mathrm{TBAPF}_{6}$ as electrolyte, scan rates $200 \mathrm{mV} / \mathrm{s}$ (cyclic voltammetry) and $5 \mathrm{mV} / \mathrm{s}$ (linear sweep). 


\section{EPR Spectroscopy}

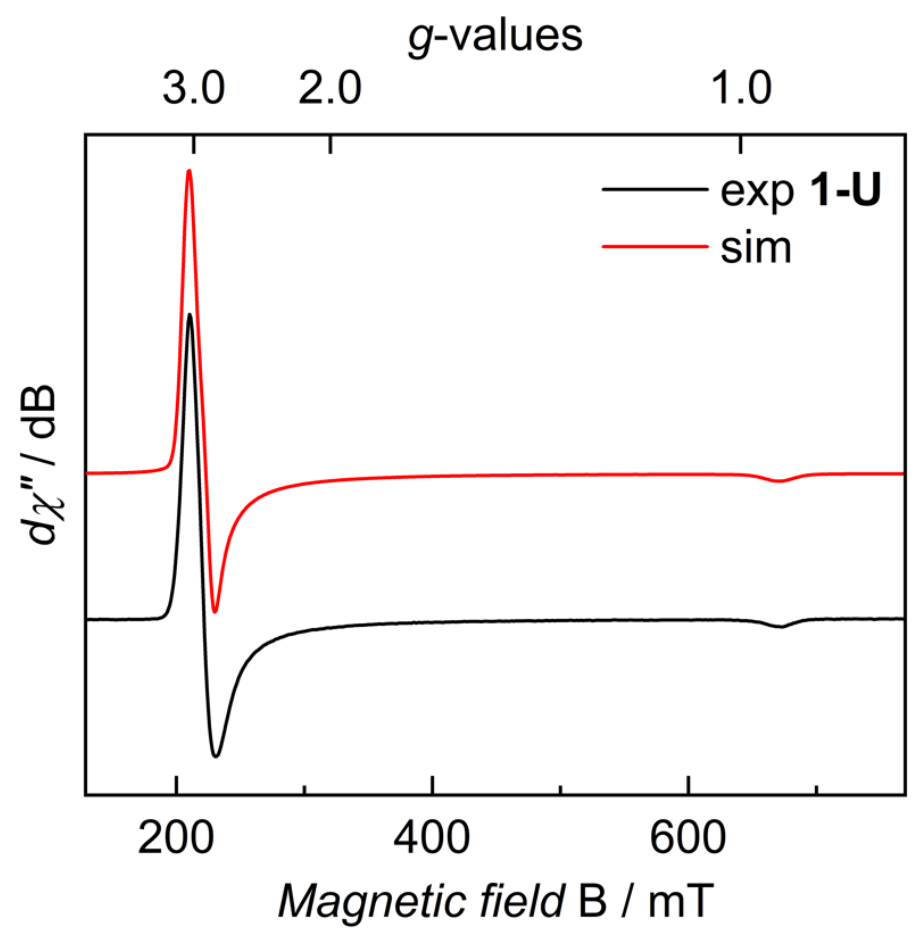

Figure S37: $\mathrm{CW} X$-Band EPR spectrum of complex 1-U, recorded at $6 \mathrm{~K}$ as a powder $(v=8.96776 \mathrm{GHz}, P=1.0 \mathrm{~mW}$, modulation $=0.1 \mathrm{mT})$. The spectrum was simulated with $g$ values of $g_{1}=3.06, g_{2}=2.85, g_{3}=0.96$, and line widths of $W_{1}=7.64, W_{2}=5.12$ and $W_{3}=6.22 \mathrm{mT}$.

\section{SQUID DC Magnetometry Details}

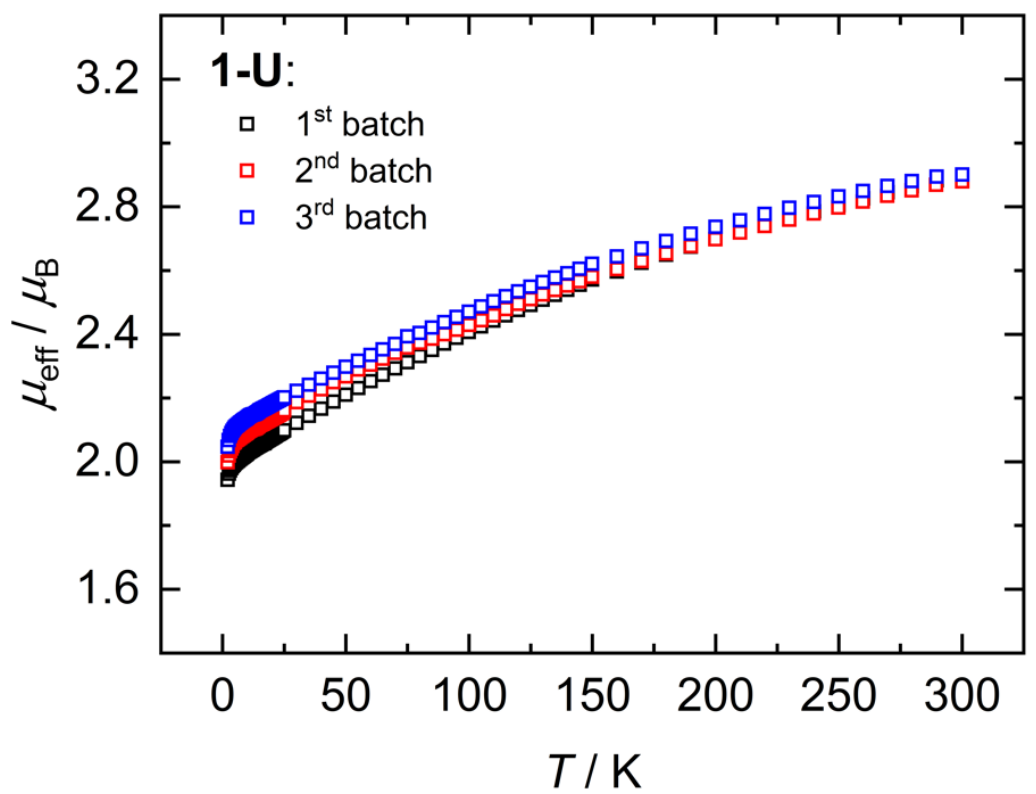

Figure S38: Temperature-dependent SQUID magnetization data $(2-300 \mathrm{~K}$ at $1 \mathrm{~T})$ for three independently synthesized samples of $\mathbf{1 - U} \mathbf{U}$, plotted as a function of the effective magnetic moment $\left(\mu_{\mathrm{eff}}\right)$ 
vs. temperature $(T)$ : sample $1(\mathrm{~m}=21.8 \mathrm{mg})$ as black squares, sample $2(\mathrm{~m}=20.2 \mathrm{mg})$ as red squares, and sample $3(\mathrm{~m}=26.1 \mathrm{mg})$ as blue squares.

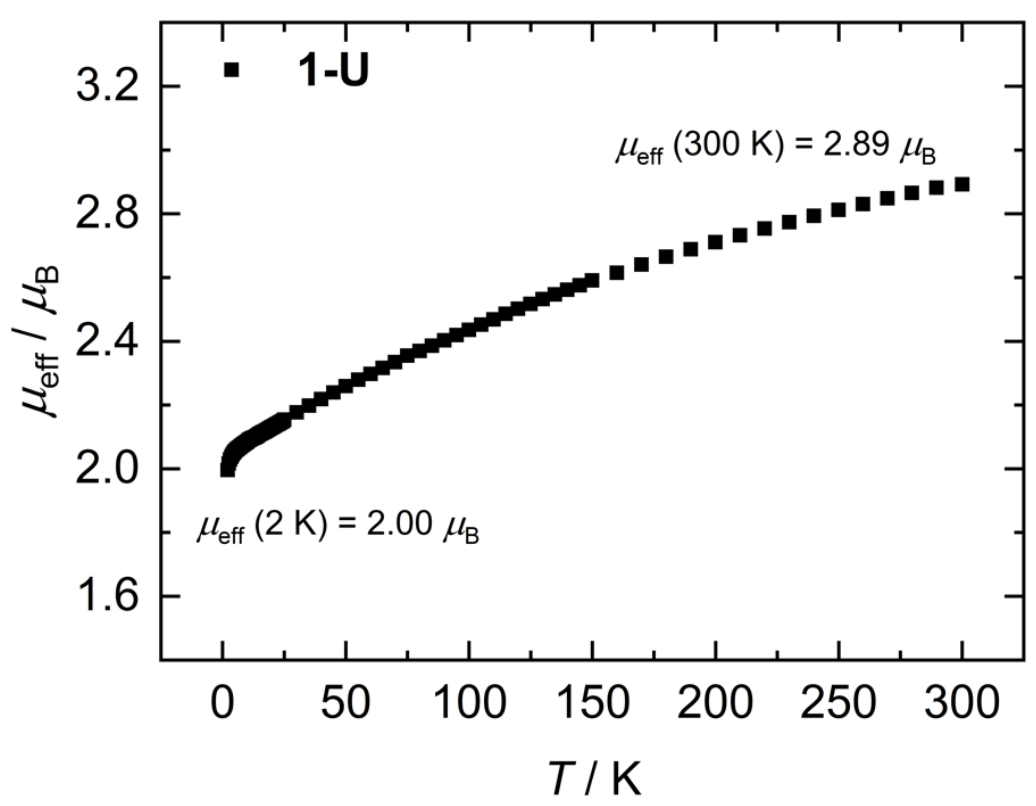

Figure S39: Temperature-dependent SQUID magnetization data $(2-300 \mathrm{~K}$ at $1 \mathrm{~T})$ for $\mathbf{1 - U}$, plotted as a function of the effective magnetic moment $\left(\mu_{\text {eff }}\right) v s$. temperature $(T)$. The depicted trace is the average over the three measurements of independently synthesized samples of 1-U. The individual results are depicted in Figure S38.

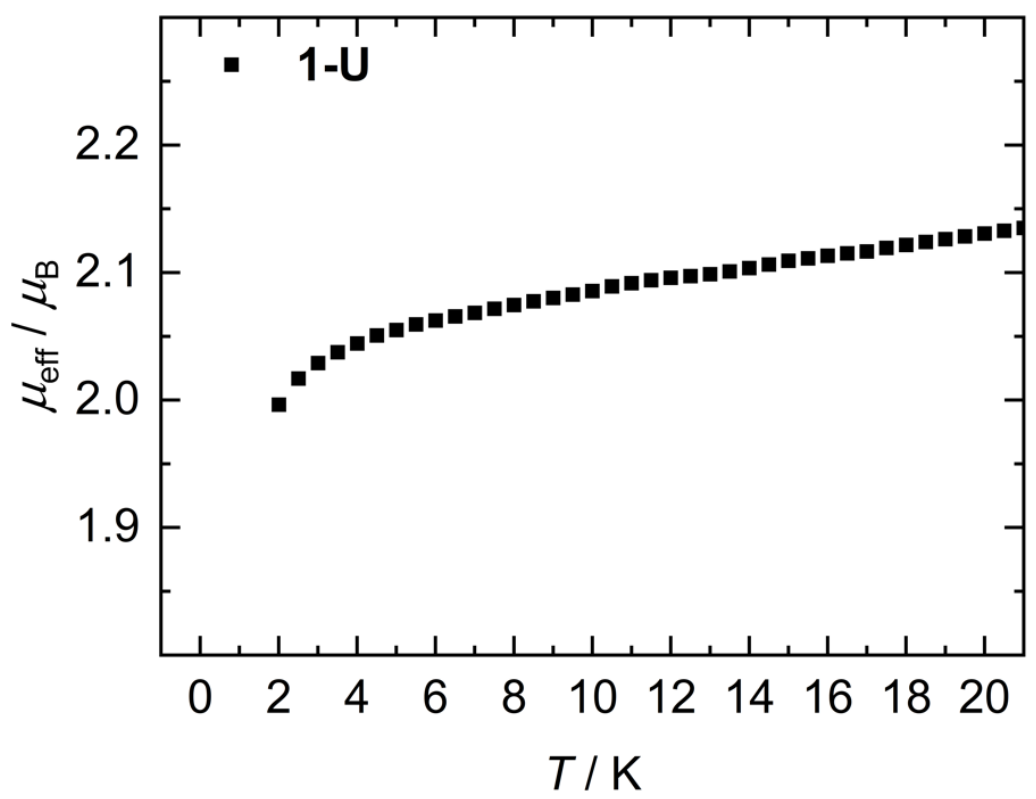

Figure S40: Zoom into the low-temperature range for the Temperature-dependent SQUID magnetization data $(2-300 \mathrm{~K}$ at $1 \mathrm{~T})$ for $\mathbf{1 - U}$, plotted as a function of the effective magnetic moment $\left(\mu_{\text {eff }}\right)$ vs. temperature $(T)$. The depicted trace is the average over the three measurements of independently synthesized samples of 1-U. The individual results are depicted in Figure S38. 

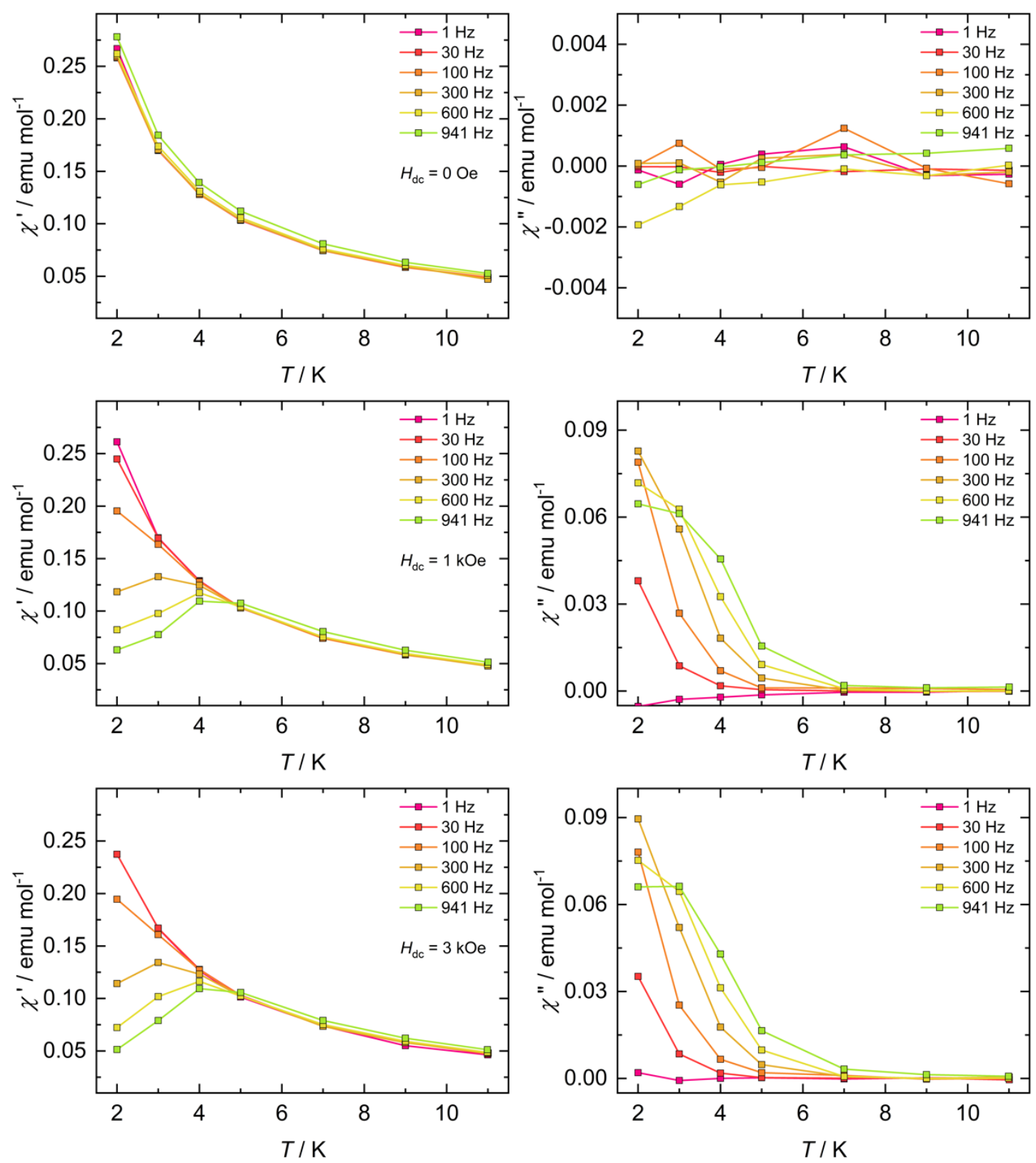

Figure S41: Plots of the in-phase $(\chi$, left $)$ and out-of-phase $(\chi$ ', right $)$ components of the ac susceptibility $v s$. temperature $T$, measured at different frequencies $\left(v_{\mathrm{ac}}=1-941 \mathrm{~Hz}\right)$, temperatures $(T=2.0-11.0 \mathrm{~K})$ and different static fields: $H_{\mathrm{dc}}=0 \mathrm{Oe}($ top), $1 \mathrm{kOe}$ (middle), $3 \mathrm{kOe}$ (bottom). 


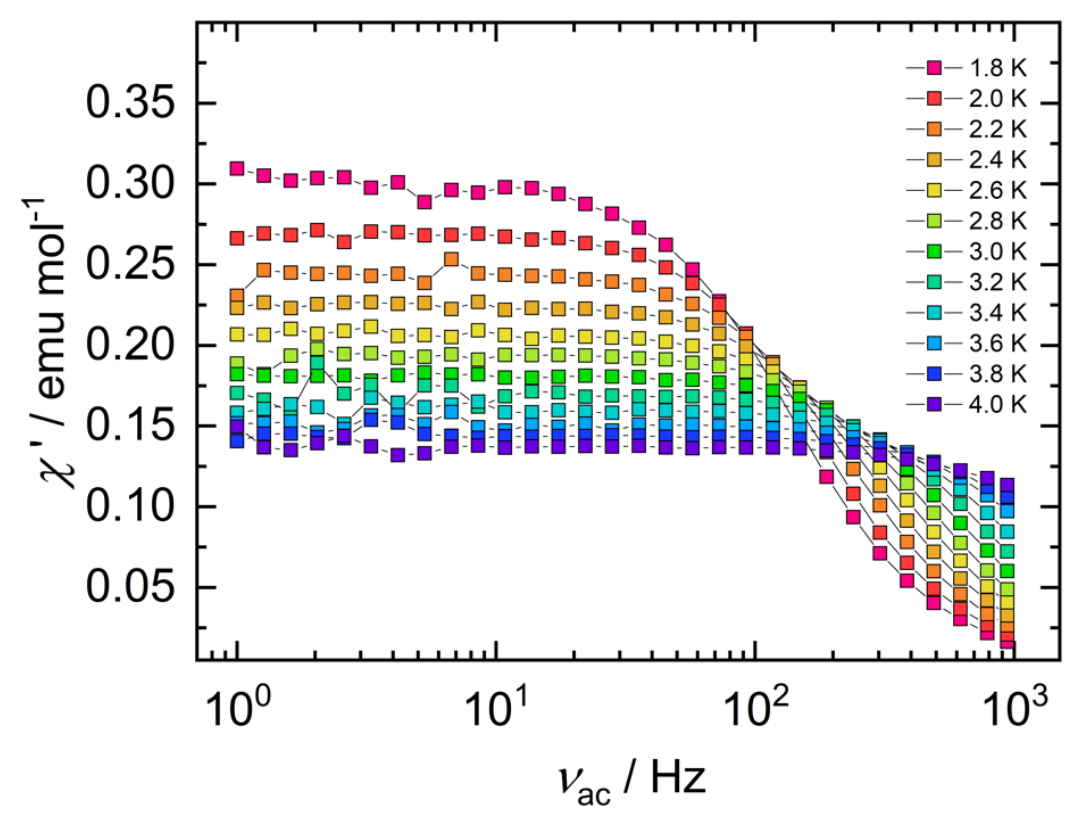

Figure S42: Plots of the in-phase $\left(\chi^{\prime}\right)$ component of the ac susceptibility $v s$. ac frequency $v_{\text {ac }}$ for solid 1-U, measured at different temperatures $(T=1.8-4.0 \mathrm{~K})$ and frequencies $\left(v_{\mathrm{ac}}=1-941 \mathrm{~Hz}\right)$ in a static field of $H_{\mathrm{dc}}=2 \mathrm{kOe}$.

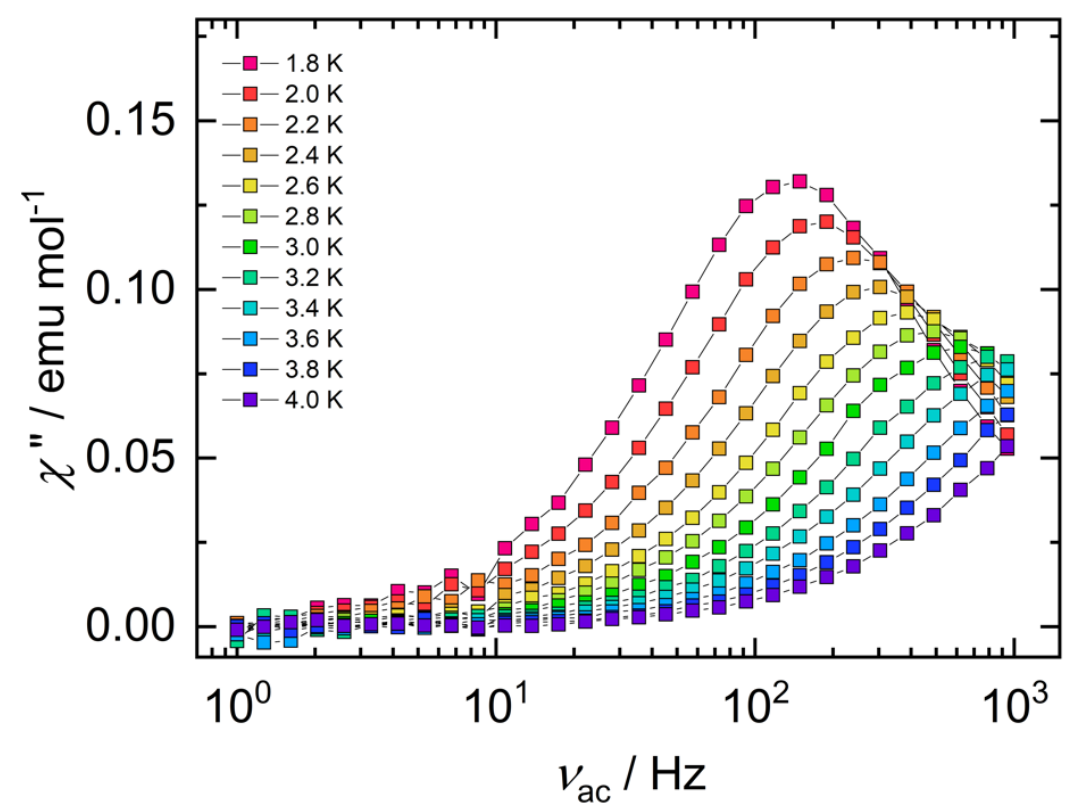

Figure S43: Plots of the out-of-phase ( $\chi$ ") component of the ac susceptibility $v s$. ac frequency $v_{\text {ac }}$ for solid 1-U, measured at different temperatures $(T=1.8-4.0 \mathrm{~K})$ and frequencies $\left(v_{\mathrm{ac}}=1-941 \mathrm{~Hz}\right)$ in a static field of $H_{\mathrm{dc}}=2 \mathrm{kOe}$. 


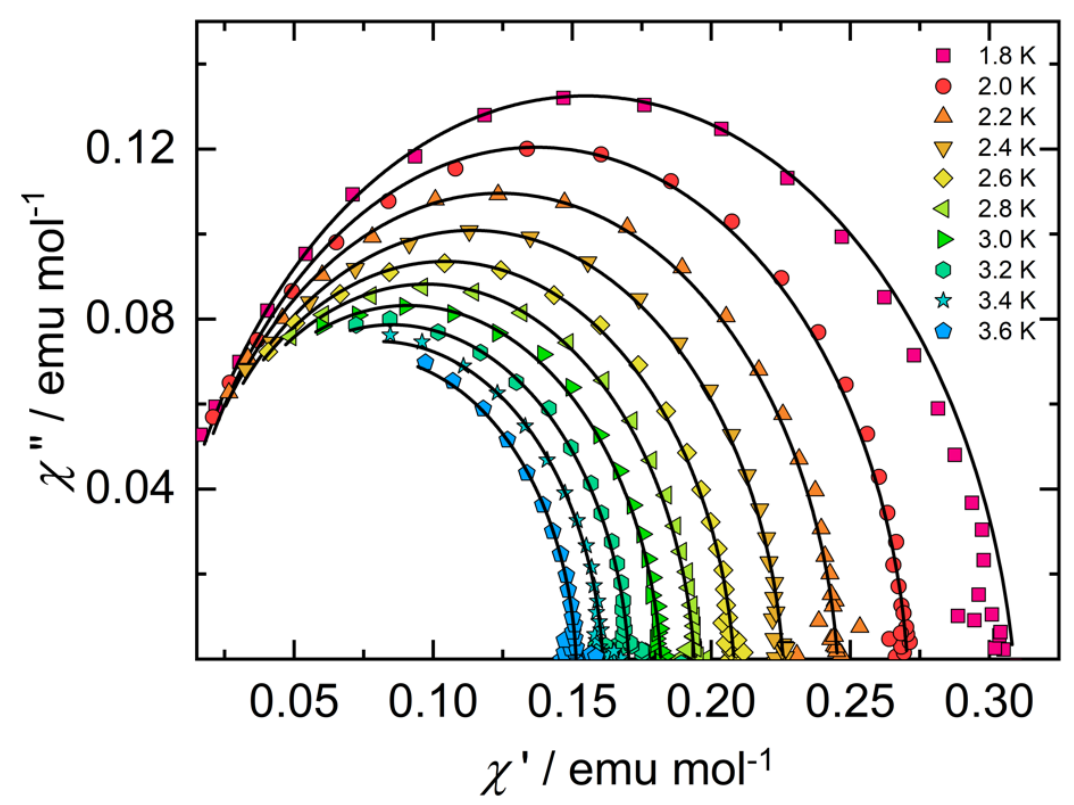

Figure S44: Cole-Cole (Argand) plots for ac susceptibility data of solid 1-U collected in a static field of $H_{\mathrm{dc}}=2 \mathrm{kOe}\left(T=1.8-3.6 \mathrm{~K}, v_{\mathrm{ac}}=1-941 \mathrm{~Hz}\right)$. The solid lines represent the best fits for a generalized Debye model (see equation nr. 1 in General Considerations, page S3).

Table S1: Generalized Debye model fitting parameters for Cole-Cole plots (Figure S44). Temperature range: $1.8-3.6 \mathrm{~K}, H_{\mathrm{dc}}=2 \mathrm{kOe}$.

\begin{tabular}{|c|c|c|c|c|}
\hline $\boldsymbol{T}[\mathbf{K}]$ & $\boldsymbol{\tau}[\mathbf{s}]$ & $\chi_{\mathbf{s}}$ & $\chi_{\mathrm{T}}$ & $\boldsymbol{\alpha}$ \\
\hline 1.8 & $1.12 \cdot 10^{-3}$ & $8.50 \cdot 10^{-4}$ & 0.30703 & 0.09513 \\
\hline 2.0 & $8.59 \cdot 10^{-4}$ & 0.00382 & 0.26281 & 0.06462 \\
\hline 2.2 & $6.60 \cdot 10^{-4}$ & 0.00247 & 0.24041 & 0.06522 \\
\hline 2.4 & $5.23 \cdot 10^{-4}$ & 0.00223 & 0.22135 & 0.06522 \\
\hline 2.6 & $4.10 \cdot 10^{-4}$ & $7.37 \cdot 10^{-4}$ & 0.20662 & 0.06516 \\
\hline 2.8 & $3.32 \cdot 10^{-4}$ & 0.00106 & 0.19172 & 0.05619 \\
\hline 3.0 & $2.63 \cdot 10^{-4}$ & $8.82 \cdot 10^{-15}$ & 0.18181 & 0.05623 \\
\hline 3.2 & $2.07 \cdot 10^{-4}$ & $4.20 \cdot 10^{-17}$ & 0.17032 & 0.05035 \\
\hline 3.4 & $1.65 \cdot 10^{-4}$ & $3.53 \cdot 10^{-17}$ & 0.16096 & 0.04697 \\
\hline 3.6 & $1.28 \cdot 10^{-4}$ & $1.11 \cdot 10^{-17}$ & 0.15138 & 0.03919 \\
\hline
\end{tabular}




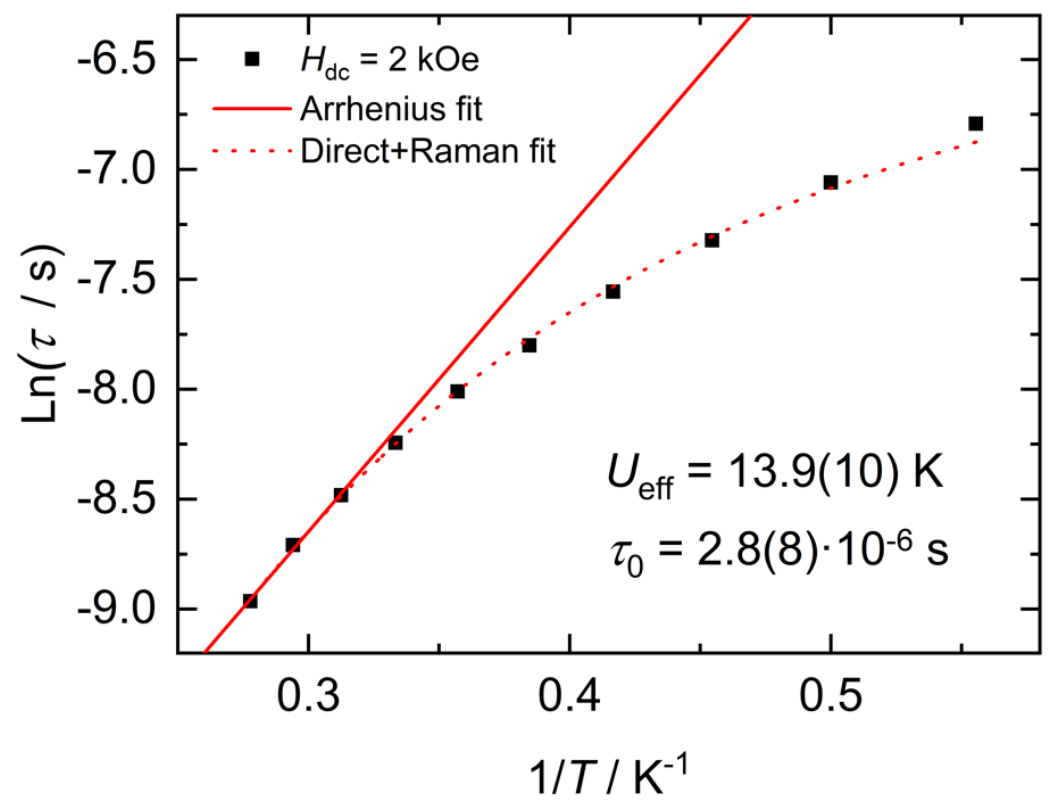

Figure S45: Plot of $1 / T v s . \ln (\tau)$ obtained from the best fit values to the Cole-Cole plots (Figure S44) reported in Table S1. The solid red line represents the best fit to the Arrhenius law $\tau=\tau_{0} \exp \left(U_{\text {eff }} / k_{B} T\right)$ (valid at the thermally activated regime) at the highest measured temperatures of the Cole-Cole plots $(T=3.2-3.6 \mathrm{~K})$. The dotted red line represents the best fit to a combination of Direct relaxation and Raman effect (see Figure S46).

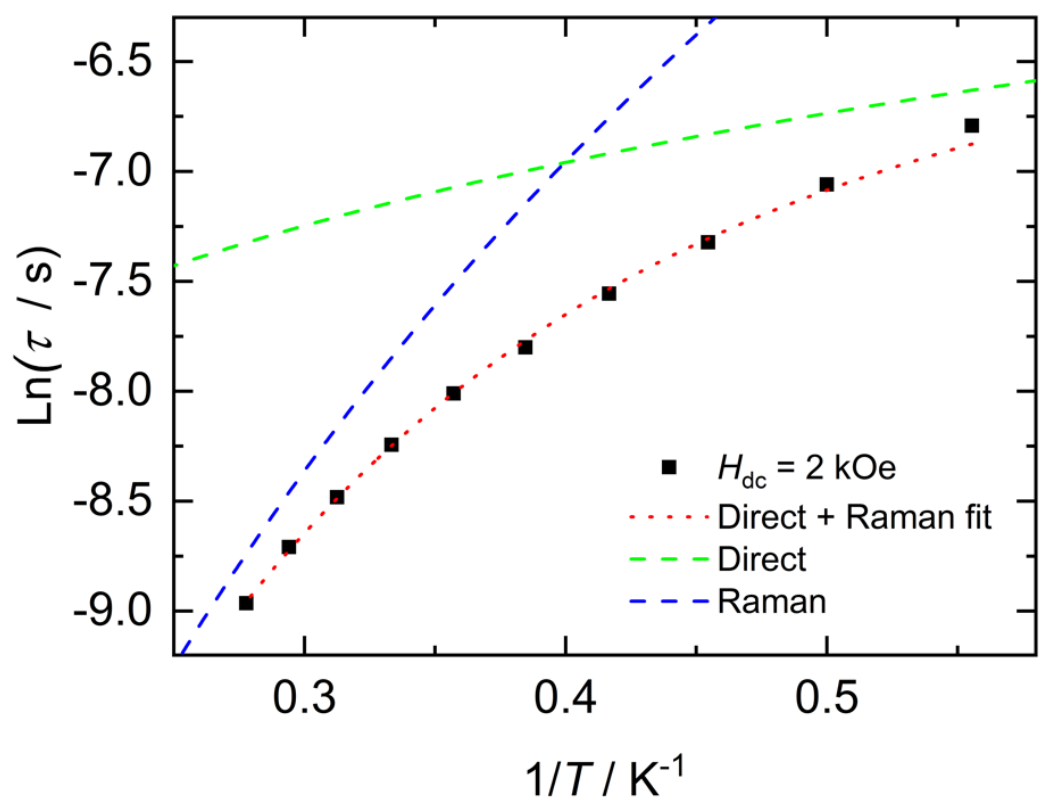

Figure S46: Plot of $1 / T v s . \ln (\tau)$ obtained from the best fit values to the Cole-Cole plots (Figure S44) reported in Table S1. Green and blue dashed lines represent individual direct and Raman fits, respectively. The dotted red line represents the best fit to a combination of direct relaxation and Raman effect, $\tau^{-1}=A T+C T^{n}$ (fit parameters: $A=420(40) \mathrm{s}^{-1} \mathrm{~K}^{-1}, C=11.8(33) \mathrm{s}^{-1} \mathrm{~K}^{-\mathrm{n}}, n=4.9(2)$; (see equation nr. 2 in General Considerations, page S3). 


\section{X-Ray Crystal Structure Determinations}

CCDC-2099530 for 1, CCDC- 2099531 for 1-U, CCDC-2099532 for 1-U-MeCN, and CCDC-2099533 for 1-U-DME contain the supplementary crystallographic data for this paper. The data can be obtained free of charge from Cambridge Crystallographic Data Centre, 12 Union Road, Cambridge, CB2 1EZ, UK (fax: ++44-1223-336-033; e-mail: deposit@ccdc.cam.ac.uk).

Compound 1. The compound crystallized with a total of 1.34 molecules of THF and 0.66 molecules of $n$-pentane per formula unit. Two crystallographic sites are shared by these two different solvent molecules. The refined site occupancies were 68.2(4) and 31.8(4) \% for the atoms O100-C104 and O111 -C115; and 65.3(6) and 34.7(6) \% for the atoms O200 - C204 and C211 - C215, respectively. Similarity and pseudo-isotropic restraints were applied in the refinement of the anisotropic displacement parameters of these disordered solvent molecules. In part, DFIX restraints were applied in the refinement of the solvent molecules. The positions of the sulphur bound hydrogen atoms were taken from a difference Fourier synthesis, and these hydrogens were allowed to ride on their carrier S atoms.

Compound 1-U. The complex molecule is located on a crystallographic threefold rotation axis and, therefore, exhibits crystallographically imposed $C_{3}$ symmetry. The compound crystallized with four molecules of cyclohexane per formula unit. While one solvent molecule was located on a general position the second independent cyclohexane was located on a crystallographic threefold rotation axis.

Compound 1-U-MeCN. The compound crystallized with one molecule of acetonitrile per formula unit. This solvent molecule was disordered. Four different orientations were refined and resulted in the following site occupancies: $53.3(5) \%$ for the atoms $\mathrm{N} 100-\mathrm{C} 102,22.3(4) \%$ for the atoms $\mathrm{N} 110-$ $\mathrm{C} 112,14.1(4) \%$ for the atoms N120 - C122, and 10.7(4) \% for the atoms N130 - C132, respectively. Similarity and pseudo-isotropic restraints were applied to the anisotropic displacement parameters of the disordered atoms.

Compound 1-U-DME. The compound crystallized with two independent molecules of the complex and two independent DME solvent molecules in its asymmetric unit. One of the DME solvent molecules was disordered. Two alternative orientations were refined and resulted in site occupancies of 51(2) and 49(2) \% for the affected atoms C301, O302 and C311, O312, respectively. Similarity and pseudoisotropic restraints were applied to the anisotropic displacement parameters of the disordered and a few other atoms. 
Table S2: Crystallographic data, data collection, and refinement details for 1, 1-U, 1-U-MeCN, and 1-U-DME.

\begin{tabular}{|c|c|c|c|c|}
\hline & 1 & 1-U & 1-U-MeCN & 1-U-DME \\
\hline CCDC-Nr. & CCDC-2099530 & CCDC- 2099531 & CCDC-2099532 & CCDC-2099533 \\
\hline Identification code & dmp2001 & dmp2103 & dmp2003 & dmp2104 \\
\hline Empirical formula & $\begin{array}{c}\mathrm{C}_{63} \mathrm{H}_{78} \mathrm{~S}_{3} \\
\cdot 1.34\left(\mathrm{C}_{4} \mathrm{H}_{8} \mathrm{O}\right) \\
\cdot 0.66\left(\mathrm{C}_{5} \mathrm{H}_{12}\right) \\
\end{array}$ & $\begin{array}{c}\mathrm{C}_{63} \mathrm{H}_{75} \mathrm{~S}_{3} \mathrm{U} \\
\cdot 4\left(\mathrm{C}_{6} \mathrm{H}_{12}\right)\end{array}$ & $\begin{array}{c}\mathrm{C}_{67} \mathrm{H}_{81} \mathrm{~N}_{2} \mathrm{~S}_{3} \mathrm{U} \\
\cdot \mathrm{C}_{2} \mathrm{H}_{3} \mathrm{~N}\end{array}$ & $\begin{array}{c}\mathrm{C}_{67} \mathrm{H}_{85} \mathrm{O}_{2} \mathrm{~S}_{3} \mathrm{U} \\
\cdot \mathrm{C}_{4} \mathrm{H}_{10} \mathrm{O}_{2}\end{array}$ \\
\hline Formula weight & 1075.66 & 1503.06 & 1289.60 & 1346.67 \\
\hline Crystal shape, color & Block, colorless & $\begin{array}{c}\text { Hexagonal prism, } \\
\text { dark blue/violet }\end{array}$ & Prism, brown & Plate, brown \\
\hline Crystal size [mm] & $0.30 \times 0.16 \times 0.14$ & $0.18 \times 0.14 \times 0.04$ & $0.25 \times 0.22 \times 0.09$ & $0.16 \times 0.08 \times 0.04$ \\
\hline Temperature $[\mathrm{K}]$ & 200 & 100 & 100 & 100 \\
\hline Crystal system & Triclinic & Trigonal & Monoclinic & Triclinic \\
\hline Space group & $P \overline{1}($ Nr. 2) & $P \overline{3}$ (Nr. 147) & $P 2_{1} / n$ (Nr. 14$)$ & $P \overline{1}(\mathrm{Nr} .2)$ \\
\hline$a[\AA]$ & $13.4154(14)$ & $16.3874(8)$ & $16.5502(9)$ & $11.6743(12)$ \\
\hline$b[\AA]$ & $15.2497(14)$ & $16.3874(8)$ & $17.3522(9)$ & $21.226(2)$ \\
\hline$c[\AA]$ & $16.3203(17)$ & $15.7184(10)$ & $20.7008(11)$ & $25.290(3)$ \\
\hline$\alpha\left[^{\circ}\right]$ & $82.798(5)$ & 90 & 90 & $105.811(4)$ \\
\hline$\beta\left[^{\circ}\right]$ & $71.468(8)$ & 90 & $97.479(2)$ & $91.060(4)$ \\
\hline$\gamma\left[^{\circ}\right]$ & $82.548(6)$ & 120 & 90 & $97.563(4)$ \\
\hline$V\left[\AA^{3}\right]$ & $3126.4(6)$ & $3655.6(4)$ & $5894.3(5)$ & $5967.6(11)$ \\
\hline$Z$ & 2 & 2 & 4 & 4 \\
\hline$\rho\left[\mathrm{g} \mathrm{cm}^{-3}\right]$ (calc.) & 1.143 & 1.366 & 1.453 & 1.499 \\
\hline$\mu\left[\mathrm{mm}^{-1}\right]$ & 0.161 & 2.351 & 2.905 & 2.876 \\
\hline$F(000)$ & 1171 & 1570 & 2636 & 2772 \\
\hline$T_{\min } ; T_{\max }$ & $0.716 ; 0.746$ & $0.438 ; 0.746$ & $0.634 ; 0.746$ & $0.627 ; 0.746$ \\
\hline $2 \Theta$ interval $\left[^{\circ}\right]$ & $3.4 \leq 2 \Theta \leq 52.8$ & $3.8 \leq 2 \Theta \leq 59.1$ & $3.8 \leq 2 \Theta \leq 61.0$ & $3.4 \leq 2 \Theta \leq 57.4$ \\
\hline Coll. Refl. & 136988 & 95822 & 260819 & 231523 \\
\hline Indep. Refl.; $R_{\text {int }}$ & $12763 ; 0.052$ & $6851 ; 0.134$ & $17985 ; 0.027$ & $30845 ; 0.070$ \\
\hline Obs. refl. $F_{0} \geq 4 \sigma(F)$ & 9951 & 5940 & 17013 & 26056 \\
\hline No. ref. param. & 792 & 276 & 782 & 1463 \\
\hline $\mathrm{wR}_{2}$ (all data) & 0.1697 & 0.0864 & 0.0412 & 0.0860 \\
\hline $\mathrm{R}_{1}\left(F_{0} \geq 4 \sigma(F)\right)$ & 0.0557 & 0.0386 & 0.0165 & 0.0450 \\
\hline GooF on $F^{2}$ & 1.044 & 1.093 & 1.073 & 1.209 \\
\hline$\Delta \rho_{\max / \min }\left[\mathrm{e} \AA^{-3}\right]$ & $0.646 ;-0.384$ & $1.018 ;-1.328$ & $0.871 ;-0.545$ & $1.868 ;-3.980$ \\
\hline
\end{tabular}




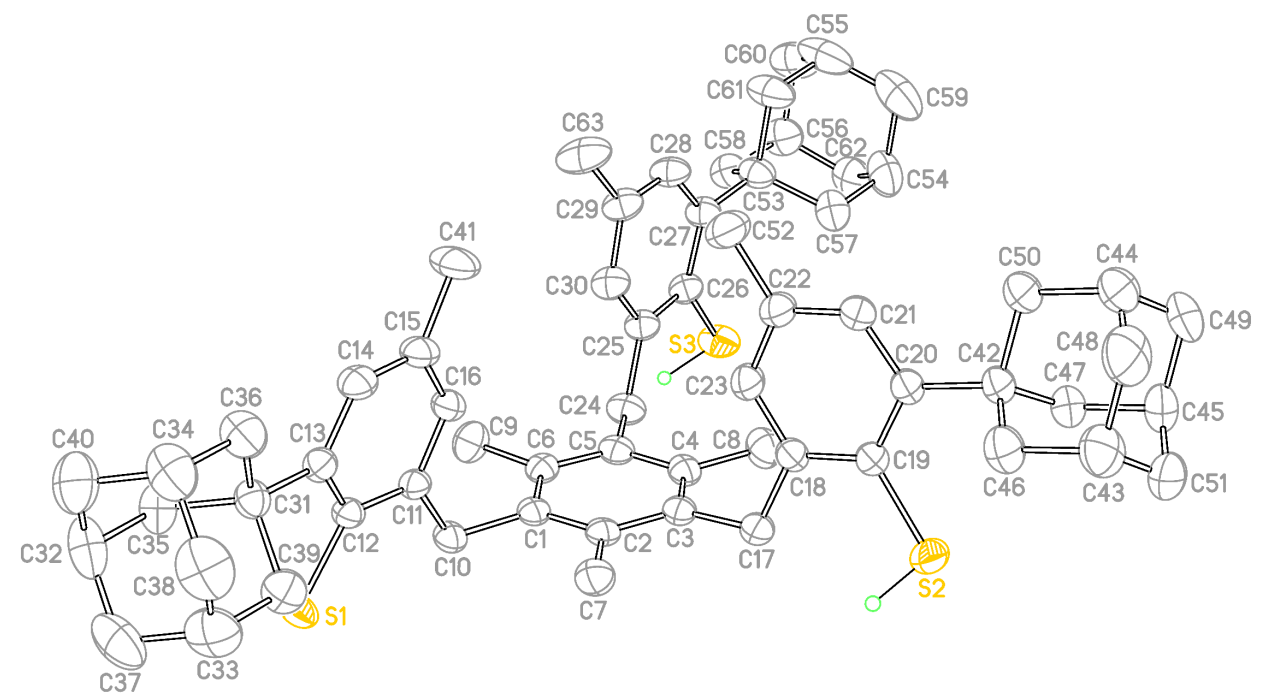

Figure S47: Molecular structure of $\mathbf{1}$ in crystals of $\mathbf{1} \cdot 1.34 \mathrm{C}_{4} \mathrm{H}_{8} \mathrm{O} \cdot 0.66 \mathrm{C}_{5} \mathrm{H}_{12}$ with the applied numbering scheme (thermal ellipsoids at 50\% probability). Hydrogen atoms (except for $S$-bound $\mathrm{H}$ atoms) and co-crystallized solvent omitted for clarity.

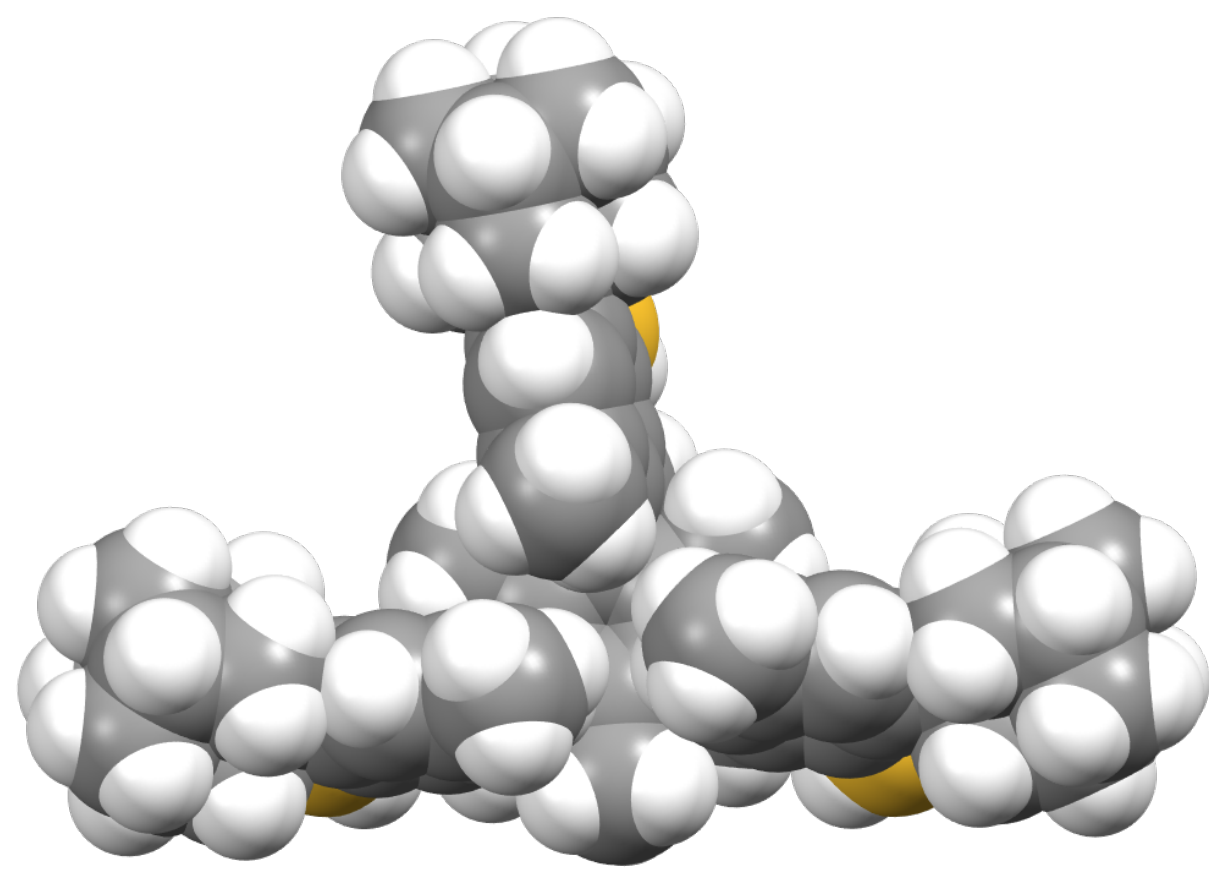

Figure S48: Top view of the space-filling model of $\mathbf{1}$ in crystals of $\mathbf{1} \cdot 1.34 \mathrm{C}_{4} \mathrm{H}_{8} \mathrm{O} \cdot 0.66 \mathrm{C}_{5} \mathrm{H}_{12}$ (Figure S47). Co-crystallized solvent omitted for clarity. 


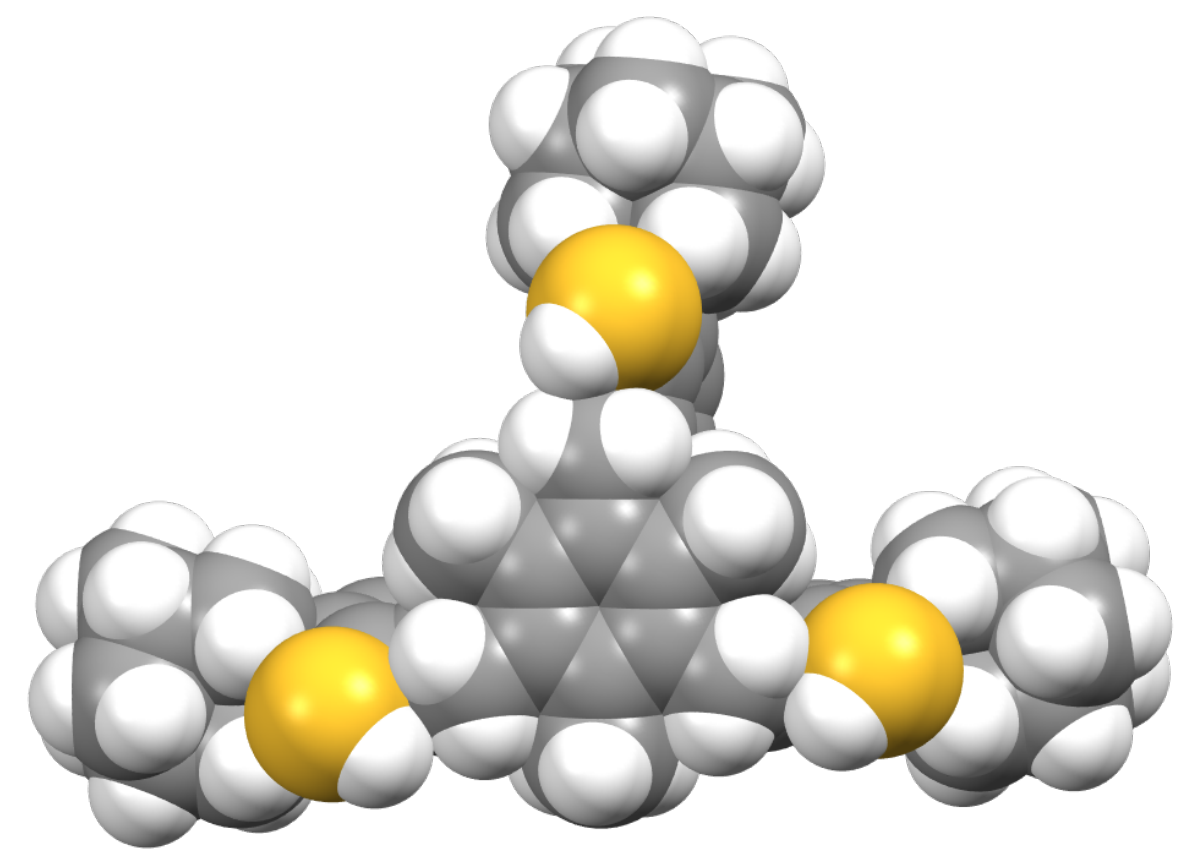

Figure S49: Bottom view of the space-filling model of $\mathbf{1}$ in crystals of $\mathbf{1} \cdot 1.34 \mathrm{C}_{4} \mathrm{H}_{8} \mathrm{O} \cdot 0.66 \mathrm{C}_{5} \mathrm{H}_{12}$ (Figure S47). Co-crystallized solvent omitted for clarity.

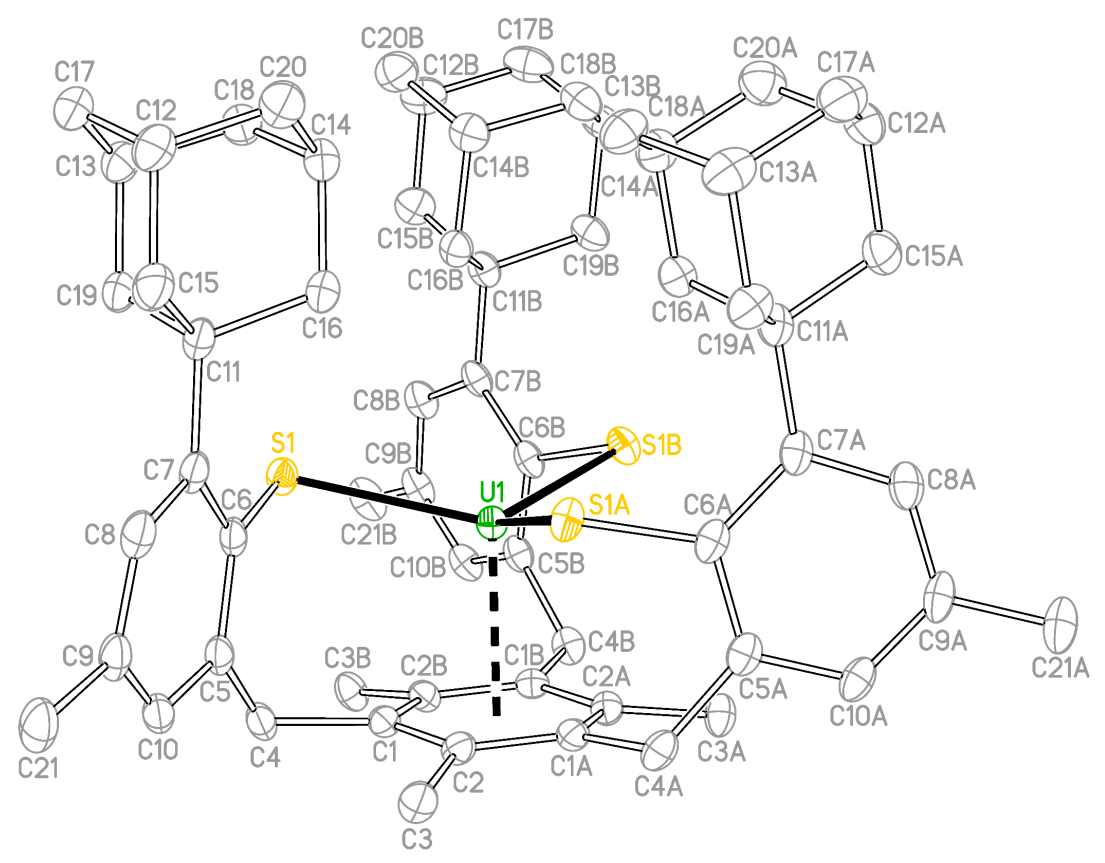

Figure S50: Molecular structure of 1-U in crystals of 1-U • $4 \mathrm{C}_{6} \mathrm{H}_{12}$ with the applied numbering scheme (thermal ellipsoids at 50\% probability). Hydrogen atoms and co-crystallized solvent omitted for clarity. 


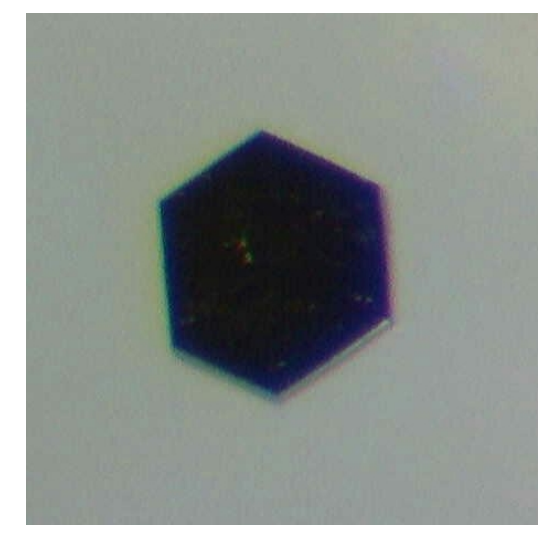

Figure S51: A single crystal of 1-U $\bullet 4 \mathrm{C}_{6} \mathrm{H}_{12}$ obtained by cyclohexane layering on a toluene solution of $1-\mathrm{U}$ at $-30{ }^{\circ} \mathrm{C}$.

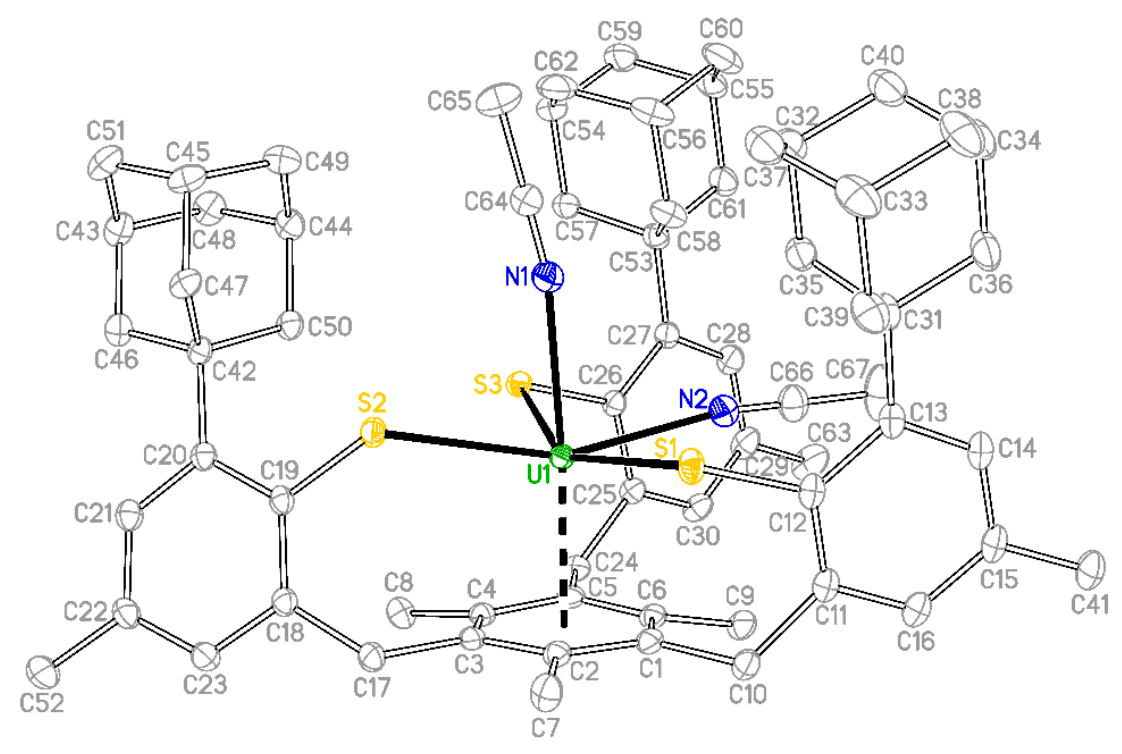

Figure S52: Molecular structure of 1-U-MeCN in crystals of 1-U-MeCN $\bullet \mathrm{C}_{2} \mathrm{H}_{3} \mathrm{~N}$ with the applied numbering scheme (thermal ellipsoids at 50\% probability). Hydrogen atoms and co-crystallized solvent omitted for clarity.

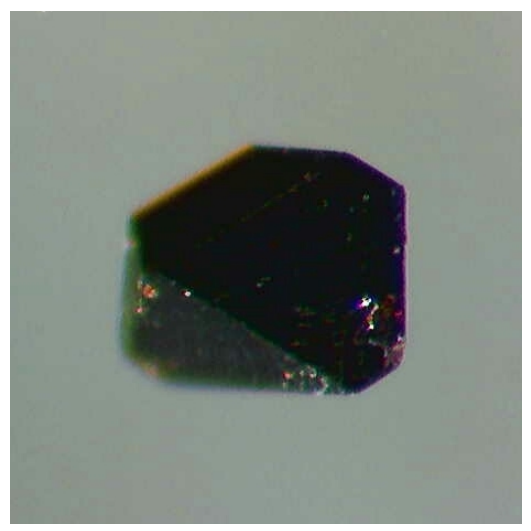

Figure S53: A single crystal of 1-U-MeCN $\cdot \mathrm{C}_{2} \mathrm{H}_{3} \mathrm{~N}$ obtained by slow crystallization from an acetonitrile solution of $\mathbf{1 - U}$ at RT. 

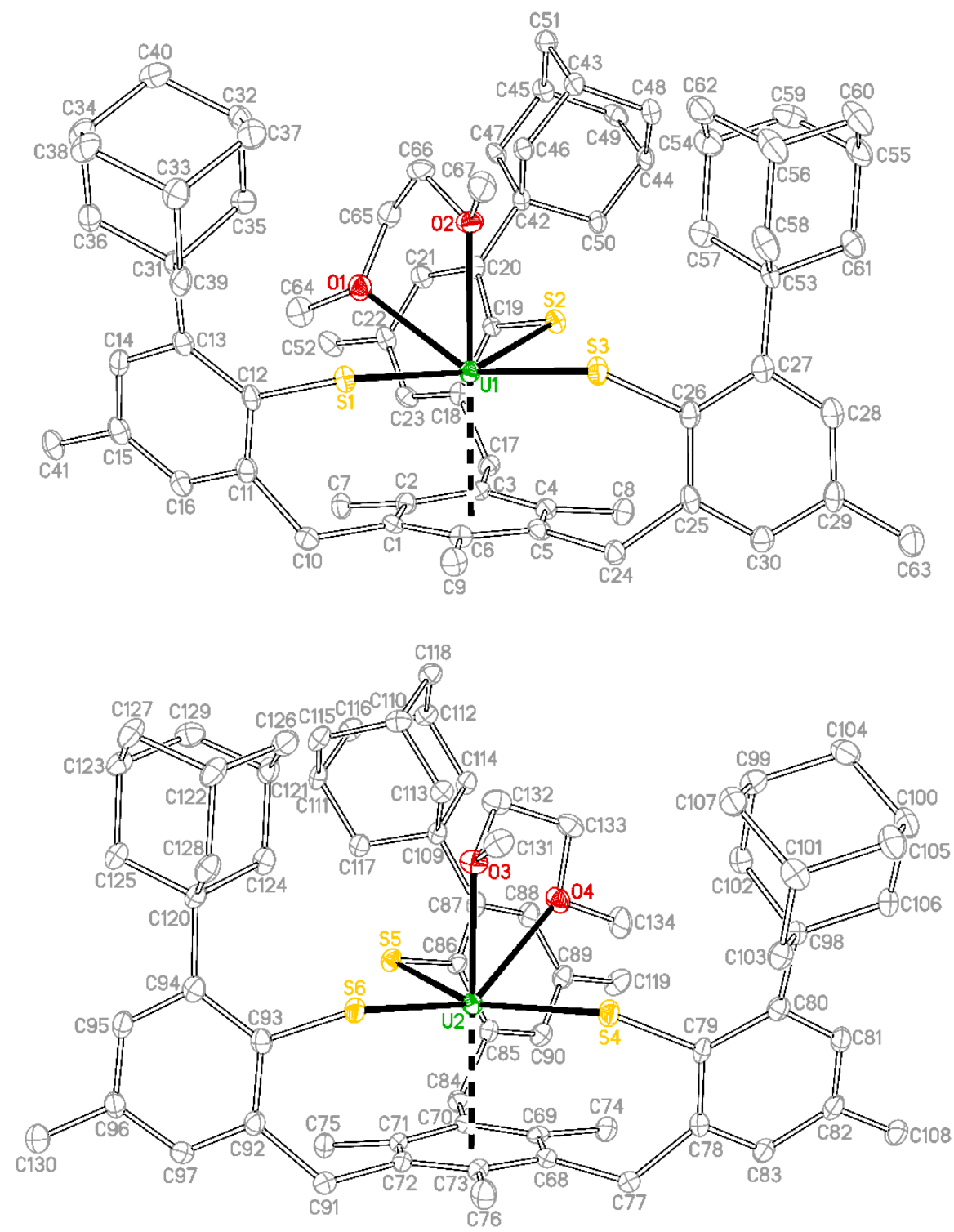

Figure S54: Molecular structures of the two independent molecules of 1-U-DME in crystals of 1-U-DME $-\mathrm{C}_{4} \mathrm{H}_{10} \mathrm{O}_{2}$ with the applied numbering scheme (thermal ellipsoids at $50 \%$ probability). Hydrogen atoms and co-crystallized solvent omitted for clarity.

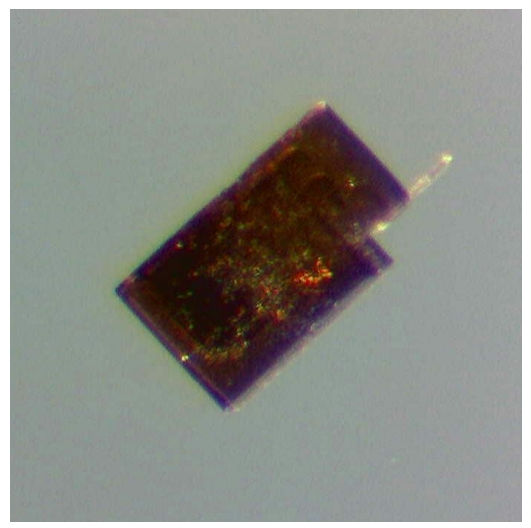

Figure S55: A single crystal of 1-U-DME $\cdot \mathrm{C}_{4} \mathrm{H}_{10} \mathrm{O}_{2}$ obtained by slow crystallization over 2-3 weeks from a 1,2-dimethoxyethane solution of $\mathbf{1}-\mathbf{U}$ at $-30{ }^{\circ} \mathrm{C}$. 
Table S3: Selected bond lengths $(\AA)$ and angles $\left(^{\circ}\right)$ for complexes 1-U, 1-U-MeCN, 1-U-DME, and 1-U-THF. [a] oop is defined as the out-of-plane shift of the uranium ion from the plane defined by the chalcogenide atoms.

\begin{tabular}{|c|c|c|c|}
\hline $\begin{array}{l}\text { structural } \\
\text { parameter }\end{array}$ & $1-U$ & 1-U-MeCN & 1-U-DME \\
\hline \multirow{2}{*}{ U-S } & \multirow{2}{*}{$2.7082(7)$} & \multirow{2}{*}{$\begin{array}{l}2.8137(4)(\mathrm{U} 1-\mathrm{S} 1), \\
2.7377(3)(\mathrm{U} 1-\mathrm{S} 2), \\
2.7865(3)(\mathrm{U} 1-\mathrm{S} 3)\end{array}$} & $\begin{array}{l}2.8356(10)(\mathrm{U} 1-\mathrm{S} 1), \\
2.8588(10)(\mathrm{U} 1-\mathrm{S} 2), \\
2.7509(10)(\mathrm{U} 1-\mathrm{S} 3)\end{array}$ \\
\hline & & & $\begin{array}{l}2.8376(11)(\mathrm{U} 2-\mathrm{S} 4), \\
2.8425(11)(\mathrm{U} 2-\mathrm{S} 5) \\
2.7513 \text { (10) (U2-S6) }\end{array}$ \\
\hline \multirow{2}{*}{ U-solv } & \multirow{2}{*}{$\mathrm{n} / \mathrm{a}$} & \multirow{2}{*}{$\begin{array}{l}2.5999(12)(\mathrm{U} 1-\mathrm{N} 1) \\
2.5831(12)(\mathrm{U} 1-\mathrm{N} 2)\end{array}$} & $\begin{array}{l}2.598(3)(\mathrm{U} 1-\mathrm{O} 1), \\
2.565(3)(\mathrm{U} 1-\mathrm{O} 2)\end{array}$ \\
\hline & & & $\begin{array}{l}2.596(3)(\mathrm{U} 2-\mathrm{O} 3), \\
2.624(3)(\mathrm{U} 2-\mathrm{O} 4)\end{array}$ \\
\hline \multirow{2}{*}{ Ar-S } & \multirow{2}{*}{$1.798(3)$} & \multirow{2}{*}{$\begin{array}{l}1.7805(13)(\mathrm{S} 1-\mathrm{C} 12), \\
1.7781(13)(\mathrm{S} 2-\mathrm{C} 19), \\
1.7784(13)(\mathrm{S} 3-\mathrm{C} 26)\end{array}$} & $\begin{array}{l}1.788(4)(\mathrm{S} 1-\mathrm{C} 12), \\
1.783(4)(\mathrm{S} 2-\mathrm{C} 19), \\
1.796(4)(\mathrm{S} 3-\mathrm{C} 26)\end{array}$ \\
\hline & & & $\begin{array}{l}1.788(4)(\mathrm{S} 4-\mathrm{C} 79), \\
1.786(4)(\mathrm{S} 5-\mathrm{C} 86), \\
1.788(4)(\mathrm{S} 6-\mathrm{C} 93)\end{array}$ \\
\hline \multirow{2}{*}{$\Varangle\left(\mathrm{U}-\mathrm{S}-\mathrm{C}_{i p s o}\right)$} & \multirow{2}{*}{$92.31(9)$} & \multirow{2}{*}{$\begin{array}{l}106.86(4)(\mathrm{U} 1-\mathrm{S} 1-\mathrm{C} 12), \\
119.70(4)(\mathrm{U} 1-\mathrm{S} 2-\mathrm{C} 19), \\
105.91(4)(\mathrm{U} 1-\mathrm{S} 3-\mathrm{C} 26)\end{array}$} & $\begin{array}{l}\text { 108.01(13) (U1-S1-C12), } \\
104.98(12)(\mathrm{U} 1-\mathrm{S} 2-\mathrm{C} 19), \\
115.17(14)(\mathrm{U} 1-\mathrm{S} 3-\mathrm{C} 26)\end{array}$ \\
\hline & & & $\begin{array}{l}120.36(14) \text { (U2-S4-C79), } \\
105.29(14)(\mathrm{U} 2-\mathrm{S} 5-\mathrm{C} 86), \\
109.81(13)(\mathrm{U} 2-\mathrm{S} 6-\mathrm{C} 93)\end{array}$ \\
\hline \multirow{2}{*}{$\Varangle(S-U-S)$} & \multirow{2}{*}{$115.949(11)$} & \multirow{2}{*}{$\begin{array}{c}108.150(10)(\mathrm{S} 1-\mathrm{U} 1-\mathrm{S} 2) \\
150.064(10)(\mathrm{S} 1-\mathrm{U} 1-\mathrm{S} 3) \\
91.874(10)(\mathrm{S} 2-\mathrm{U} 1-\mathrm{S} 3)\end{array}$} & $\begin{array}{c}\text { 161.03(3) (S1-U1-S2), } \\
93.23(3)(\mathrm{S} 1-\mathrm{U} 1-\mathrm{S} 3), \\
96.86(3)(\mathrm{S} 2-\mathrm{U} 1-\mathrm{S} 3)\end{array}$ \\
\hline & & & $\begin{array}{l}\text { 156.96(3) (S4-U2-S5), } \\
\text { 95.24(3) (S4-U2-S6), } \\
\text { 103.98(3) (S5-U2-S6) }\end{array}$ \\
\hline \multirow{2}{*}{$\Varangle(\mathrm{S}-\mathrm{U}-\mathrm{S})_{\text {sum }}$} & \multirow{2}{*}{$347.85(3)$} & \multirow{2}{*}{$350.09(3)$} & 351.12(9)(U1) \\
\hline & & & 356.18(9) (U2) \\
\hline \multirow{2}{*}{$\mathrm{U}_{\text {oop }}^{[\mathrm{a}]}$} & \multirow{2}{*}{$-0.5525(8)$} & \multirow{2}{*}{$-0.4470(2)$} & $-0.3606(7)$ (U1) \\
\hline & & & $-0.2642(7)$ (U2) \\
\hline \multirow{2}{*}{ U-mes centroid } & \multirow{2}{*}{2.464} & \multirow{2}{*}{2.462} & $2.502(\mathrm{U} 1)$ \\
\hline & & & 2.541 (U2) \\
\hline \multirow{2}{*}{ mes $\mathrm{C}-\mathrm{C}$} & \multirow{2}{*}{$\begin{array}{l}1.420(4)(\mathrm{C} 1-\mathrm{C} 2), \\
1.415(4)(\mathrm{C} 1-\mathrm{C} 2 \mathrm{~B})\end{array}$} & \multirow{2}{*}{$\begin{array}{l}1.4220(17)(\mathrm{C} 1-\mathrm{C} 2), \\
1.4250(18)(\mathrm{C} 2-\mathrm{C} 3), \\
1.4175(17)(\mathrm{C} 3-\mathrm{C} 4), \\
1.4239(17)(\mathrm{C} 4-\mathrm{C} 5), \\
1.4173(18)(\mathrm{C} 5-\mathrm{C} 6), \\
1.4207(18)(\mathrm{C} 1-\mathrm{C} 6)\end{array}$} & $\begin{array}{l}1.418(5)(\mathrm{C} 1-\mathrm{C} 2), \\
1.422(5)(\mathrm{C} 2-\mathrm{C} 3), \\
1.418(5)(\mathrm{C} 3-\mathrm{C} 4), \\
1.413(5)(\mathrm{C} 4-\mathrm{C} 5), \\
1.430(5)(\mathrm{C} 5-\mathrm{C} 6), \\
1.423(6)(\mathrm{C} 1-\mathrm{C} 6)\end{array}$ \\
\hline & & & $\begin{array}{l}1.407(6)(\mathrm{C} 68-\mathrm{C} 69), \\
1.418(6)(\mathrm{C} 69-\mathrm{C} 70), \\
1.421(5)(\mathrm{C} 70-\mathrm{C} 71), \\
1.405(6)(\mathrm{C} 71-\mathrm{C} 72), \\
1.430(5)(\mathrm{C} 72-\mathrm{C} 73), \\
1.418(5)(\mathrm{C} 68-\mathrm{C} 73)\end{array}$ \\
\hline
\end{tabular}




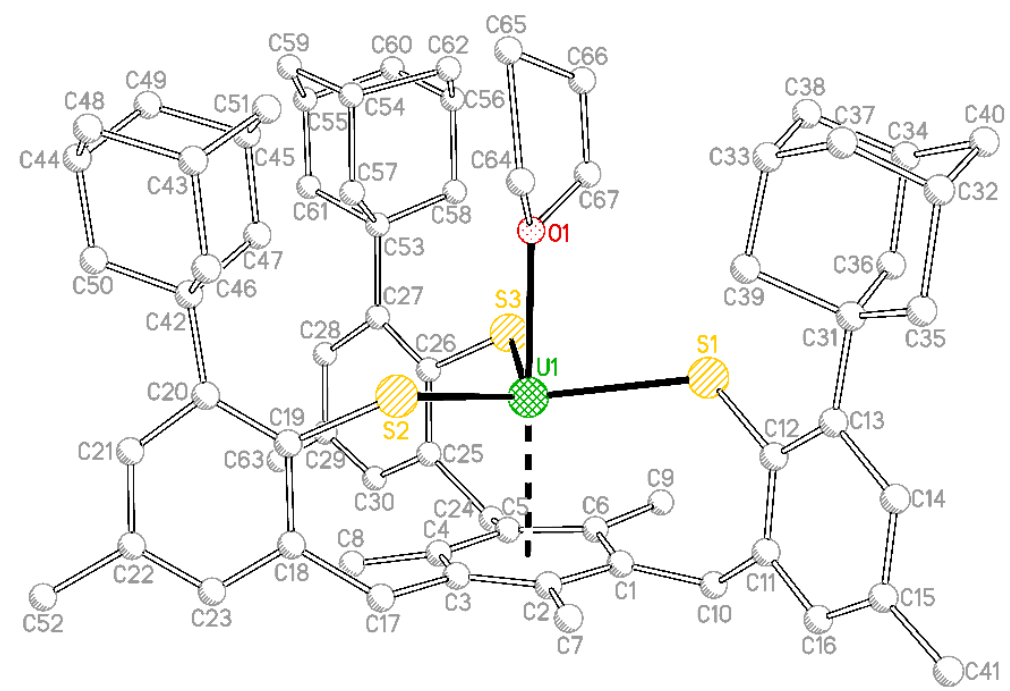

Figure S56: Ball-and-stick model with the applied numbering scheme of 1-U-THF, in crystals obtained by pentane diffusion into a THF solution of 1-U at RT. Hydrogen atoms and co-crystallized solvent omitted for clarity. The crystal and data quality were rather poor and thus only sufficient for a qualitative structure solution (non-hydrogen atoms could be refined with isotropic displacement parameters only).

\section{Further Computational Results}

The Pipek Mezey method localizes molecular orbitals with the constraint to maximize orbital dependent maximal charges. The received orbitals do not diagonalize the Fock matrix and, hence, have no orbitalenergy values attached to them. The orbitals are visualized in Figure S57, and the order is the one obtained from the calculation, which was performed with Orca. The MO's were visualized with gabedit.

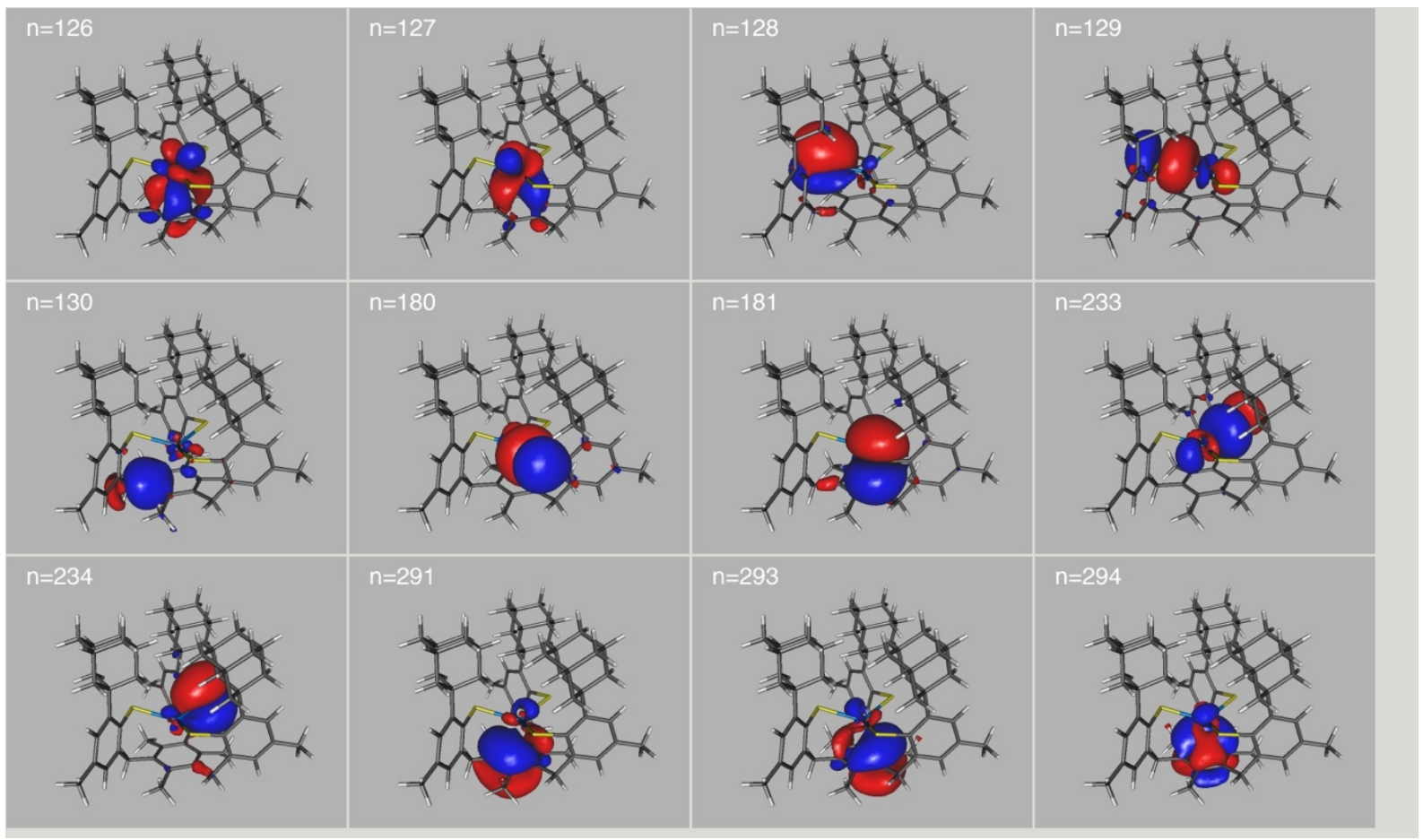

Figure S57: Localized molecular orbitals containing U-ligand contributions. The localization method used is due to Pipek and Mezey. The numbering of the MO's is arbitrary. 
In Figure S58, the molecular graph for $\mathbf{1 - U}$ is shown as computed by the QTAIM method. Visualization and calculations were done with the AIMALL code based on scalar-relativistic all-electron DFT calculation. It is interesting to note, that this graph shows only three bond-critical points for the Umesitylene interactions. In a smaller system, in which the adamantyl and methyl groups are removed to decrease computational demands, six such interactions are found. This change in system size does not affect the QTAIM values significantly. For comparison, the molecular graph of the smaller system is shown in Figure S59 for 1-U and for the related oxygen compound [ $\left.\mathrm{U}^{\mathrm{III}}\left(\left(\mathrm{OAr}{ }^{\mathrm{Ad}, \mathrm{Me}}\right)_{3} \mathrm{mes}\right)\right]$.

In Table S4, the most important QTAIM metrics are given for 1-U. The DI values are related to a number of parameters that can be calculated in different ways, known as bond orders. Two of these values, namely the Mayer and Wiberg bond orders are given in Table S4. The Wiberg bond order is the older concept and can be seen as a somewhat simplified version of the Mayer bond order. The Mayer bond order itself can be viewed as a sort of delocalization index in Hilbert space. Both bond orders give similar results, as expected. It can be seen that changes in similar bonds are reproduced by the bond orders when compared to the DI. It should be noted, however, that DI's and bond orders between different bond types should not be compared (see e.g., the DI's and BO's for U-S vs. C-C).

The smaller systems presented in Figure S59 were used both in the CAS calculations for the EPR parameters, as well as for the IQA calculations. In the IQA framework, the total energy is partitioned into atomic self-energy terms and interaction terms. These pairwise interactions energies consist of nucleus-nucleus, nucleus-electron, and electron-electron interactions. The last part is of interest in the definition of the bond between an atomic pair. It can be further broken down into a coulomb part and an exchange-correlation part. These two parts define the ionic and covalent contribution to the electronic interaction energy. IQA calculations are very time consuming; to expedite the calculations, the smaller model systems (Figure S59) were used. To check the validity of this approach, the QTAIM values for 1-U and the model systems were compared, and no large deviations were found. IQA values are given in Table S5.

Within the framework of conceptual DFT, it is possible to calculate quantities that are related to reactivity. These global indices of reactivity include the chemical potential, hardness and softness, and the electrophilicity index. In principle, these can be calculated using vertical ionization and electron attachment energies. Using Koopmans' theorem, it is possible to simplify these calculations even further and use values for the frontier orbitals instead.

$$
\begin{gathered}
\mu \approx \frac{E_{H O M O}+E_{L U M O}}{2} \\
\eta \approx E_{L U M O}-E_{H O M O} \\
S=\frac{1}{\eta}
\end{gathered}
$$




$$
\omega=\frac{\mu^{2}}{2 \cdot \eta}
$$

Values for the global indices of reactivity based on this procedure are given in Table S6. These are global indices and do not reflect the local reactivity at the uranium center. For example, the chemical potential can be seen as the negative of the molecular electronegativity. The calculated values suggest that the U-S compound is slightly more electronegative than the $\mathrm{U}-\mathrm{O}$ compound. The given values are best used to explain reactivities in a series of related molecules.

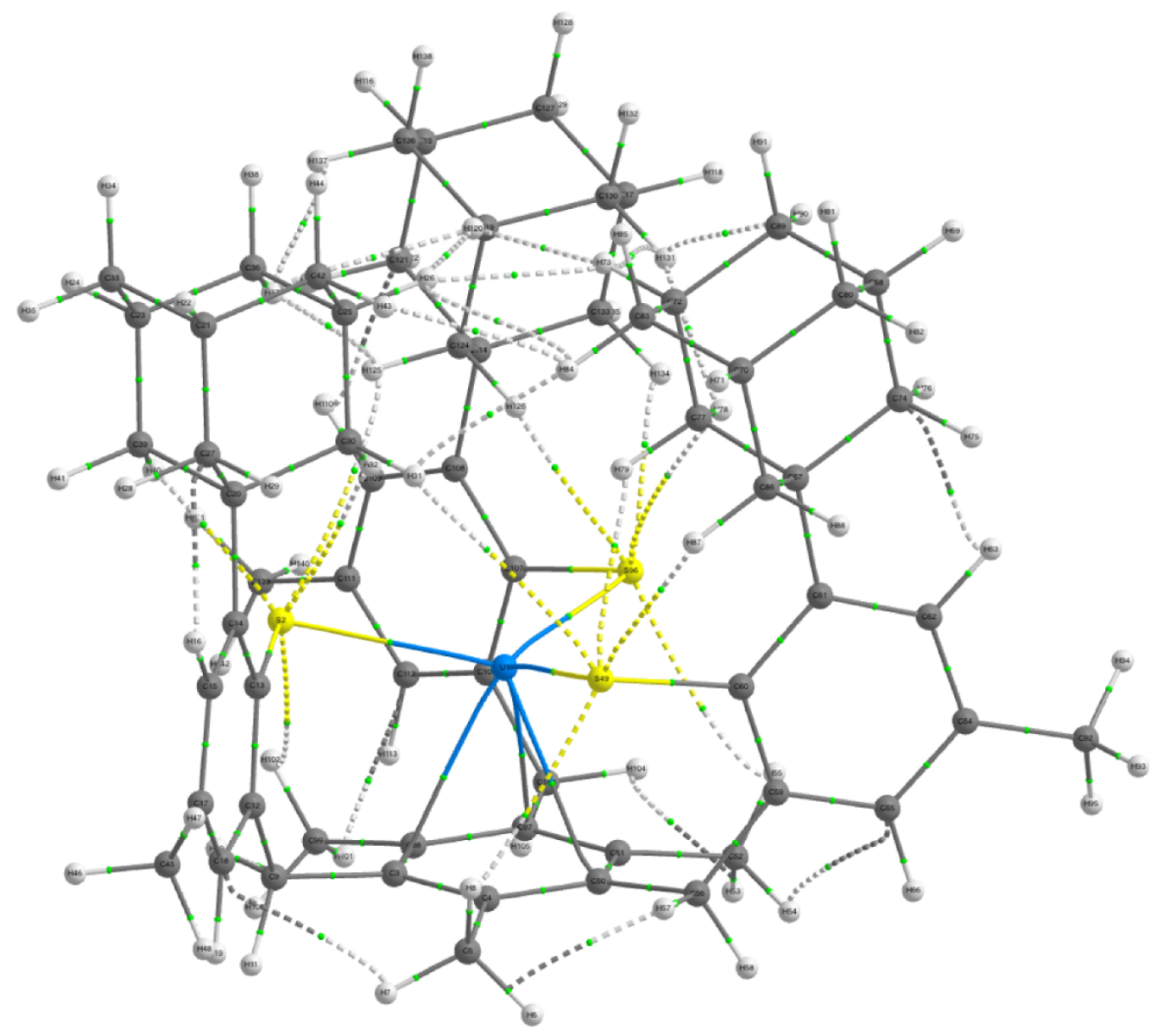

Figure S58: Molecular graph of 1-U from a QTAIM calculation. 

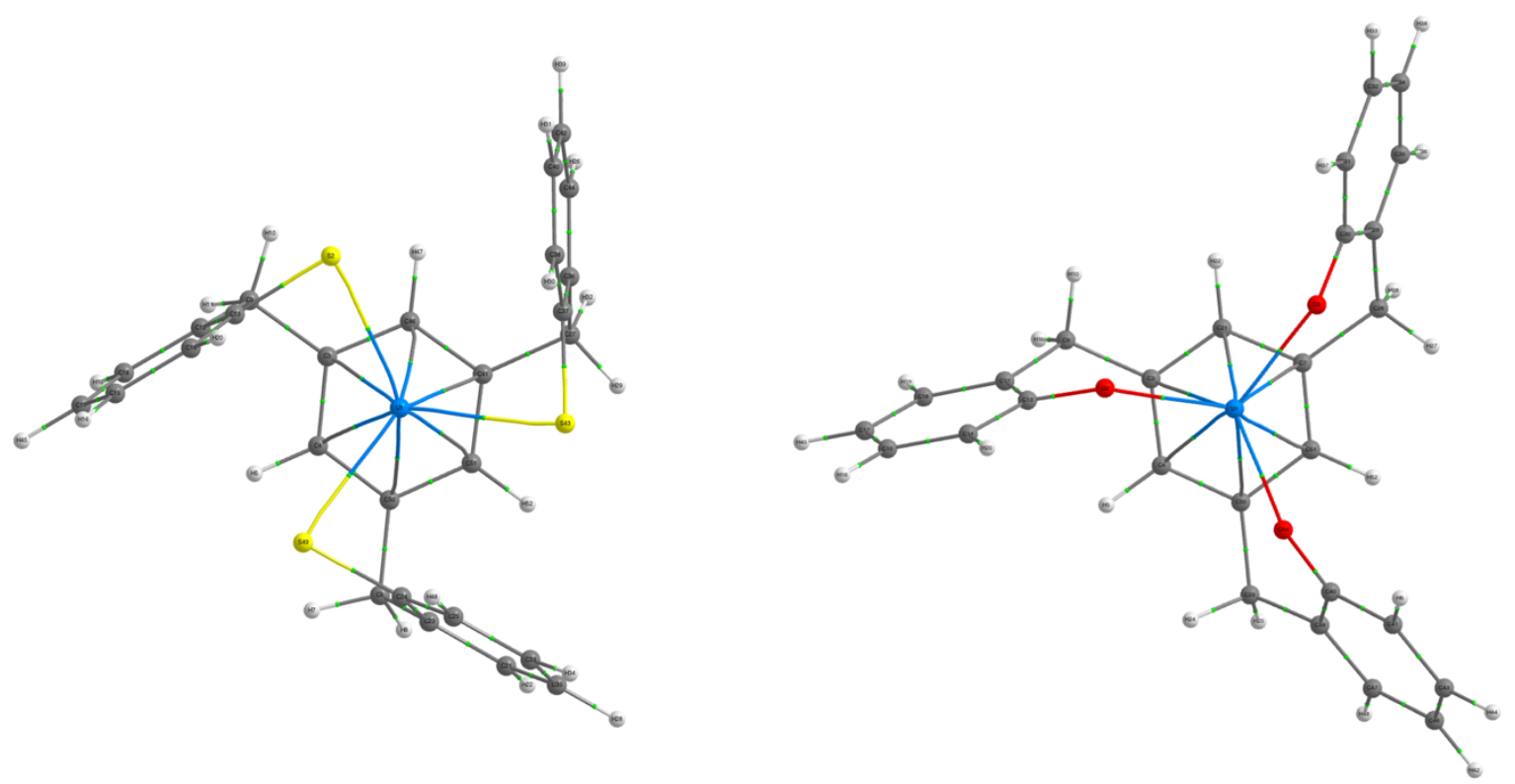

Figure S59: Molecular graphs of the model compounds used in IQA and CAS calculations: 1-U (left) and $\left[\mathrm{U}^{\mathrm{III}}\left(\left(\mathrm{OAr}{ }^{\mathrm{Ad}, \mathrm{Me}}\right)_{3} \mathrm{mes}\right)\right]$ (right).

Table S4: Selected QTAIM Values and bond indices for 1-U.

\begin{tabular}{|c|c|c|c|c|c|}
\hline \multirow{2}{*}{ Atoms } & \multicolumn{3}{|c|}{ QTAIM } & \multicolumn{2}{|c|}{ Bond Indices } \\
\hline & $\mathrm{DI}(\mathbf{A} \mid \mathbf{B})$ & Rho & $\mathbf{H}=\mathbf{V}+\mathbf{G}$ & Mayer & Wiberg \\
\hline $\mathrm{U}-\mathrm{S} 1$ & 0.649447 & 0.068227 & -0.017439 & 0.9739 & 0.9662 \\
\hline $\mathrm{U}-\mathrm{S} 2$ & 0.649986 & 0.068273 & -0.017471 & 0.9779 & 0.9703 \\
\hline $\mathrm{U}-\mathrm{S} 3$ & 0.65052 & 0.068359 & -0.017498 & 0.9845 & 0.9764 \\
\hline $\mathrm{U}-\mathrm{C}$ (ring) 1 & 0.203456 & 0.038035 & -0.003687 & 0.2344 & 0.2315 \\
\hline $\mathrm{U}-\mathrm{C}$ (ring) 3 & 0.203905 & 0.038207 & -0.003769 & 0.2412 & 0.2394 \\
\hline $\mathrm{U}-\mathrm{C}$ (ring) 5 & 0.20214 & 0.038237 & -0.003825 & 0.2285 & 0.2242 \\
\hline $\begin{array}{c}\mathrm{C} 1-\mathrm{C} 2 \\
\text { (ring) }\end{array}$ & 1.291881 & 0.307735 & -0.321153 & 1.0451 & 1.0459 \\
\hline $\begin{array}{c}\mathrm{C} 2-\mathrm{C} 3 \\
\text { (ring) }\end{array}$ & 1.253431 & 0.303571 & -0.312899 & 0.9969 & 0.9874 \\
\hline $\begin{array}{c}\mathrm{C} 3-\mathrm{C} 4 \\
\text { (ring) }\end{array}$ & 1.291088 & 0.307736 & -0.321267 & 1.0567 & 1.0576 \\
\hline $\begin{array}{c}\mathrm{C} 4-\mathrm{C} 5 \\
\text { (ring) }\end{array}$ & 1.254198 & 0.303644 & -0.313139 & 0.9969 & 1.0217 \\
\hline $\begin{array}{c}\mathrm{C} 5-\mathrm{C} 6 \\
\text { (ring) }\end{array}$ & 1.290796 & 0.30768 & -0.320932 & 1.0212 & 1.1002 \\
\hline $\begin{array}{c}\mathrm{C} 6-\mathrm{C} 1 \\
\text { (ring) }\end{array}$ & 1.253881 & 0.30383 & -0.313577 & 0.9863 & 0.9977 \\
\hline
\end{tabular}


Table S5: QTAIM and IQA values for the U-S and U-O model compounds (see Figure S57).

\begin{tabular}{|c|c|c|c|c|c|c|}
\hline Atoms & \multicolumn{3}{|c|}{ QTAIM } & \multicolumn{2}{c|}{ IQA } & \\
\hline & DI(A|B) & Rho & H=V+G & EIQA(A,B) & VeeX(A,B) & \%Covalency \\
\hline $\mathrm{U}-\mathrm{S} 1$ & 0.6738 & 0.066706 & -0.014363 & -0.3115 & -0.1214 & 38.97271268 \\
\hline $\mathrm{U}-\mathrm{C}($ ring)1 & 0.218036 & 0.038071 & -0.002194 & -0.0773 & -0.0374 & 48.38292367 \\
\hline $\mathrm{U}-\mathrm{C}($ ring) 2 & 0.226861 & 0.037825 & -0.001913 & -0.0711 & -0.0385 & 54.14908579 \\
\hline $\begin{array}{c}\text { C1 - C2 } \\
\text { (ring) }\end{array}$ & 1.291615 & 0.298184 & -0.27755 & & & \\
\hline & & & & & & \\
\hline $\begin{array}{c}\mathrm{U}-\mathrm{O} 1 \\
\mathrm{U}-\mathrm{C}(\text { ring)1 }\end{array}$ & 0.721908 & 0.106961 & -0.022465 & -0.8224 & -0.1544 & 18.77431907 \\
\hline $\mathrm{U}-\mathrm{C}($ ring)2 & 0.260145 & 0.040423 & -0.00263 & -0.0404 & -0.0831 & 205.6930693 \\
\hline $\begin{array}{c}\text { C1 - C2 } \\
\text { (ring) }\end{array}$ & 1.280763 & 0.295973 & -0.273807 & & & \\
\hline
\end{tabular}

Table S6: Values from conceptual DFT; energies of the frontier orbitals E(LUMO) and E(HOMO), chemical potential $\mu$, hardness $\eta$, softness $S$ and electrophilicity $\omega$. All values are given in $\mathrm{eV}$.

\begin{tabular}{|c|c|c|}
\hline Property & $\mathbf{1 - U}$ & {$\left[\mathbf{U}^{\text {III }}\left(\left(\mathbf{O A}^{\mathbf{A d}, \mathbf{M e}}\right)_{3} \mathbf{m e s}\right)\right]$} \\
\hline $\mathbf{E}(\mathbf{L U M O})$ & -1.4118 & -1.2512 \\
\hline $\mathbf{E}(\mathbf{H O M O})$ & -4.6672 & -4.4031 \\
\hline $\boldsymbol{\mu}$ & -3.0395 & -2.8271 \\
\hline $\boldsymbol{\eta}$ & 3.2554 & 3.1519 \\
\hline $\mathbf{S}$ & 0.3072 & 0.3173 \\
\hline $\boldsymbol{\omega}$ & 1.4190 & 1.2679 \\
\hline
\end{tabular}

The spin densities shown in the main text of the article can be further analyzed and quantified. In Table $\mathrm{S}_{7}$, the NBO-based atomic populations from spin densities for $\mathrm{U}, \mathrm{S}$ and $\mathrm{O}$ are given for complexes $\left[\mathrm{U}^{\mathrm{III}}\left(\left(\mathrm{SAr}^{\mathrm{Ad}, \mathrm{Me}}\right)_{3} \mathrm{mes}\right)\right](\mathbf{1}-\mathrm{U})$ and $\left[\mathrm{U}^{\mathrm{III}}\left(\left(\mathrm{OAr}^{\mathrm{Ad}, \mathrm{Me}}\right)_{3} \mathrm{mes}\right)\right]$.

Table S7: Atomic populations from spin densities (NBO analysis), positive values indicate $\alpha$-excess, negative values $\beta$-excess.

\begin{tabular}{|c|c|c|c|c|c|c|}
\hline & & Total & $\mathrm{n}(\mathrm{s})$ & $n(p)$ & $n(d)$ & $n(f)$ \\
\hline \multirow{2}{*}{$\begin{array}{l}{\left[\mathrm{U}^{\mathrm{III}}\left(\left(\mathrm{SAr}{ }^{\mathrm{Ad}, \mathrm{Me}}\right)_{3} \mathrm{mes}\right)\right]} \\
(\mathbf{1}-\mathbf{U})\end{array}$} & $\mathrm{U}$ & 3.03273 & 0.04190 & 0.01509 & 0.18842 & 2.78710 \\
\hline & $\mathrm{S}$ & -0.03011 & -0.00191 & -0.02855 & 0.00033 & 0.00002 \\
\hline \multirow{2}{*}[\mathrm{U}^{\mathrm{III}}((\mathrm{OAr}{}^{\mathrm{Ad},\mathrm{Me}})_{3}\mathrm{mes})]{} & $\mathrm{U}$ & 2.93782 & 0.05761 & 0.02106 & 0.19762 & 2.66121 \\
\hline & $\mathrm{O}$ & -0.02759 & -0.00207 & -0.02560 & 0.00006 & 0.00000 \\
\hline
\end{tabular}




\section{References}

(1) Gademann, K.; Chavez, D. E.; Jacobsen, E. N. Highly Enantioselective Inverse-Electron-Demand Hetero-Diels-Alder Reactions of $\alpha, \beta$-Unsaturated Aldehydes. Angew. Chem. Int. Ed. 2002, 41 (16), 3059-3061.

(2) Monreal, M. J.; Thomson, R. K.; Cantat, T.; Travia, N. E.; Scott, B. L.; Kiplinger, J. L. UI 4 (1,4dioxane $)_{2},\left[\mathrm{UCl}_{4}(1,4 \text {-dioxane })\right]_{2}$, and $\mathrm{UI}_{3}(1,4 \text {-dioxane })_{1.5}$ : Stable and Versatile Starting Materials for Low- and High-Valent Uranium Chemistry. Organometallics 2011, 30 (7), 2031-2038.

(3) Fulmer, G. R.; Miller, A. J. M.; Sherden, N. H.; Gottlieb, H. E.; Nudelman, A.; Stoltz, B. M.; Bercaw, J. E.; Goldberg, K. I. NMR Chemical Shifts of Trace Impurities: Common Laboratory Solvents, Organics, and Gases in Deuterated Solvents Relevant to the Organometallic Chemist. Organometallics 2010, 29 (9), 2176-2179.

(4) Bain, G. A.; Berry, J. F. Diamagnetic Corrections and Pascal's Constants. J. Chem. Educ. 2008, 85 (4), 532-536.

(5) Coutinho, J. T.; Perfetti, M.; Baldoví, J. J.; Antunes, M. A.; Hallmen, P. P.; Bamberger, H.; Crassee, I.; Orlita, M.; Almeida, M.; van Slageren, J.; Pereira, L. C. J. Spectroscopic Determination of the Electronic Structure of a Uranium Single-Ion Magnet. Chem. Eur. J. 2019, 25 (7), 1758-1766.

(6) Liddle, S. T.; van Slageren, J. Improving f-element single molecule magnets. Chem. Soc. Rev. 2015, 44 (19), 6655-6669.

(7) Meihaus, K. R.; Long, J. R. Actinide-based single-molecule magnets. Dalton Trans. 2015, 44 (6), $2517-2528$.

(8) Marcó, A.; Compañó, R.; Rubio, R.; Casals, I. Assessment of Additives for Nitrogen, Carbon, Hydrogen and Sulfur Determination by Organic Elemental Analysis. Microchimica Acta 2003, 142 (12), 13-19.

(9) Eckhard Bill. EPR Program Eview, 2019.

(10) Eckhard Bill. EPR Program Esim, 2019.

(11) Gaffney, B. J.; Silverstone, H. J. Simulation of the EMR Spectra of High-Spin Iron in Proteins. In: Berliner, L. J., Reuben, J., Eds.; EMR of Paramagnetic Molecules. Biological Magnetic Resonance, Vol. 13; Springer, Boston, MA, 1993; 1-57.

(12) Krause, L.; Herbst-Irmer, R.; Sheldrick, G. M.; Stalke, D. Comparison of silver and molybdenum microfocus X-ray sources for single-crystal structure determination. J. Appl. Cryst. 2015, 48, 3-10.

(13) Sheldrick, G. M. SHELXT - integrated space-group and crystal-structure determination. Acta Cryst. 2015, A71 (Pt 1), 3-8.

(14) Sheldrick, G. M. Crystal structure refinement with SHELXL. Acta Cryst. 2015, C71, 3-8.

(15) Dolomanov, O. V.; Bourhis, L. J.; Gildea, R. J.; Howard, J. A. K.; Puschmann, H. OLEX2: a complete structure solution, refinement and analysis program. J. Appl. Cryst. 2009, 42 (2), 339-341. 